OPEN ACCESS

Edited by:

Cyriel Pennartz,

University of Amsterdam, Netherlands

Reviewed by:

Emery N. Brown,

Massachusetts Institute of Technology, United States

Jorrit Steven Montijn,

Netherlands Institute for

Neuroscience (KNAW), Netherlands

*Correspondence:

Henning M. Reimann

henning.reimann@mdc-berlin.de

Received: 02 July 2019 Accepted: 27 January 2020

Published: 19 May 2020

Citation:

Reimann HM and Niendorf T (2020) The (Un)Conscious Mouse as a Model for Human Brain Functions:

Key Principles of Anesthesia and

Their Impact on Translational Neuroimaging.

Front. Syst. Neurosci. 14:8. doi: 10.3389/fnsys.2020.00008

\section{The (Un)Conscious Mouse as a Model for Human Brain Functions: Key Principles of Anesthesia and Their Impact on Translational Neuroimaging}

\author{
Henning M. Reimann ${ }^{1 *}$ and Thoralf Niendorf ${ }^{1,2}$ \\ ${ }^{1}$ Berlin Ultrahigh Field Facility (B.U.F.F.), Max-Delbrück Center for Molecular Medicine, Helmholtz Association of German \\ Research Centers (HZ), Berlin, Germany, ${ }^{2}$ Experimental and Clinical Research Center, A Joint Cooperation Between the \\ Charité Medical Faculty and the Max-Delbrück Center for Molecular Medicine, Berlin, Germany
}

In recent years, technical and procedural advances have brought functional magnetic resonance imaging (fMRI) to the field of murine neuroscience. Due to its unique capacity to measure functional activity non-invasively, across the entire brain, fMRI allows for the direct comparison of large-scale murine and human brain functions. This opens an avenue for bidirectional translational strategies to address fundamental questions ranging from neurological disorders to the nature of consciousness. The key challenges of murine $\mathrm{fMRI}$ are: (1) to generate and maintain functional brain states that approximate those of calm and relaxed human volunteers, while (2) preserving neurovascular coupling and physiological baseline conditions. Low-dose anesthetic protocols are commonly applied in murine functional brain studies to prevent stress and facilitate a calm and relaxed condition among animals. Yet, current mono-anesthesia has been shown to impair neural transmission and hemodynamic integrity. By linking the current state of murine electrophysiology, $\mathrm{Ca}^{2+}$ imaging and $\mathrm{fMRI}$ of anesthetic effects to findings from human studies, this systematic review proposes general principles to design, apply and monitor anesthetic protocols in a more sophisticated way. The further development of balanced multimodal anesthesia, combining two or more drugs with complementary modes of action helps to shape and maintain specific brain states and relevant aspects of murine physiology. Functional connectivity and its dynamic repertoire as assessed by $\mathrm{fMRI}$ can be used to make inferences about cortical states and provide additional information about whole-brain functional dynamics. Based on this, a simple and comprehensive functional neurosignature pattern can be determined for use in defining brain states and anesthetic depth in rest and in response to stimuli. Such a signature can be evaluated and shared between labs to indicate the brain state of a mouse during experiments, an important step toward translating findings across species.

Keywords: mouse, fMRI-functional magnetic resonance imaging, anesthesia, translational mouse-human, brain functional connectivity, EEG, $\mathrm{Ca}^{2+}$ imaging, MIND signature 


\section{INTRODUCTION}

Much of our understanding of human brain functions comes from murine studies. The ease of genetic modification and other practical and financial issues have made the mouse the best-explored mammalian model organism in neuroscience. A multitude of murine protocols and repositories provide reliable benchmarks in today's brain research (including the Allen Brain Project ${ }^{1}$, the Blue Brain Project ${ }^{2}$, and the Mouse ENCODE Project ${ }^{3}$; Lein et al., 2007; Sunkin et al., 2013; Erö et al., 2018; Keller et al., 2018; Frankish et al., 2019). Still, it remains an open question to what extent, and under what conditions, findings from the mouse can be translated into an understanding of human brain functions. Although the mouse brain is not merely a miniaturized version of the human brain, its comparably small and flat neocortex exhibits striking functional and structural similarities, and the subcortical architecture is evolutionarily largely preserved (VenturaAntunes et al., 2013; Hofman, 2014; Glickfeld and Olsen, 2017; Halley and Krubitzer, 2019).

In recent years, technical and procedural advances have brought functional magnetic resonance imaging (fMRI) to the field of murine neuroscience. The unique capacity of fMRI to measure functional brain activity non-invasively and across the entire brain relies on tight neurovascular coupling, in which increased neural activity triggers local elevations in cerebral blood flow $(\mathrm{CBF})$, cerebral blood volume $(\mathrm{CBV})$, and blood oxygenation (Hamilton et al., 2010; Hall et al., 2014). Each of these hemodynamic parameters can be assessed by fMRI as a surrogate for neural activity. Blood oxygenation level-dependent (BOLD) fMRI is the most popular approach due to its high sensitivity and comparably fast acquisition times (Ogawa et al., 1990; Kim, 2018). Modern MR scanners operating at ultrahigh magnetic field strengths $(\geq 7 \mathrm{~T})$ have been tailored for use with small rodents, and can achieve a relative spatial resolution analogous to that commonly used in human fMRI (voxels per anatomical region; voxel size of $\sim 200 \mu \mathrm{m}^{3}$ ). This permits direct comparisons of large-scale murine and human brain functions, and opens up opportunities to use a plethora of genetically engineered models to clarify a wide range of clinical and basic neuroscience issues-from the pathogenesis of neurological disorders to fundamental questions about consciousness.

Although the first report on murine fMRI was published more than two decades ago (Huang et al., 1996), its application to mice continues to require extensive refinements (Mandino et al., 2020). Aside from technical issues including signal amplification from the small mouse brain or its vulnerability to physiological perturbations, a fundamental problem of interspecies translation involves the non-voluntary task conundrum. In comparison to (adult) humans, a mouse has no interest in participating in an fMRI study. Head fixation, body restraint, and habituation to the acoustic noise produced by the MR gradients (up to $115 \mathrm{~dB}$ sound pressure) cause enormous stress to this remarkably rousable

\footnotetext{
${ }^{1}$ mouse.brain-map.org

${ }^{2}$ bbp.epfl.ch/nexus/cell-atlas

${ }^{3}$ encodeproject.org
}

creature, which is far less amenable to training than rats (Jonckers et al., 2014, 2015; Low et al., 2016b; Dopfel and Zhang, 2018). Studying unbiased nociception in mice is almost impossible; even the application of aversive stimuli, like mild cooling of the paw, causes immediate withdrawal, and functional patterns across the brain reflect not only stress, fear, and anticipation, but also unrelated motor and sensory responses. This introduces tremendous complexity to fMRI of the awake mouse.

Low-dose anesthetic protocols are commonly applied in mouse fMRI to address these problems and to alleviate potential suffering due to stress, fear, and pain. The goal is to achieve high-quality translational data from sedated, i.e., calm, relaxed, and undistracted subjects. However, anesthetics have been shown to impair neural transmission (Baumgart et al., 2015; Hemmings et al., 2019) and also affect other aspects of murine physiology including hemodynamics (Franceschini et al., 2010; Masamoto and Kanno, 2012), thermoregulation (Reimann et al., 2016), respiration (van Alst et al., 2019), and cardiovascular control (Sinclair, 2003; Low et al., 2016a) in a dose-dependent manner. All these parameters can affect neurovascular coupling, which links the BOLD effect to the activity of neural populations. Therefore, anesthetic protocols in fMRI face two major challenges: first, to preserve functional brain states based on neural oscillation and transmission characteristics (see "Brain States, Anesthetic Depth, and Murine Consciousness" section), and second, to maintain hemodynamic integrity (see "Murine fMRI and Hemodynamic Integrity" section).

Recognizing the progress, opportunities, and challenges of murine functional brain mapping, this work provides a review of the literature on current anesthetic protocols and their meaning for murine functional neuroimaging. The aim is to introduce the basic principles of anesthesia to better understand and interpret the outcome of murine fMRI studies, and to develop novel anesthetic protocols and monitoring strategies dedicated to promoting reproducible and translational neuroimaging.

\section{BRAIN STATES, ANESTHETIC DEPTH, AND MURINE CONSCIOUSNESS}

In the murine as in the human brain, neural information is processed against a background of spontaneous, ongoing activity generated by the promiscuous firing of neurons or structured patterns of neural populations. These oscillatory dynamics can be assessed via invasive and non-invasive neural readout techniques, such as electroencephalography (EEG), local field potentials (LFP), or calcium $\left(\mathrm{Ca}^{2+}\right)$ imaging, yielding distinct spatiotemporal profiles that can be considered as signatures of distinct brain states.

\section{Cortical States of Wakefulness}

Awake brain states undergo constant alterations in response to changing contexts of arousal, attention, and behavior (Steriade, 2000; Pfaff, 2005; Olcese et al., 2018; Poulet and Crochet, 2019). The spectrum of EEG patterns during these states ranges from synchronous low-frequency oscillations $(<8 \mathrm{~Hz})$ during quiet wakefulness to structured higherfrequency oscillations $(\sim 8-100 \mathrm{~Hz})$ during attentional tasks 
and highly desynchronized high-frequency oscillations during aroused states (Petersen et al., 2003; Palva et al., 2005; Fries et al., 2007; Sachidhanandam et al., 2013; McGinley et al., 2015a; Olcese et al., 2018; Poulet and Crochet, 2019). The level of cortical activation (i.e., higher-frequency oscillations) is positively correlated to the level of general arousal, which is mediated by modulatory input from subcortical cholinergic and monoaminergic (dopaminergic, noradrenergic, serotonergic, and histaminergic) nuclei (Figure 1A; Aston-Jones et al., 1999; Pfaff, 2005; Harris and Thiele, 2011; Lee and Dan, 2012; McGinley et al., 2015b; Lecrux and Hamel, 2016; Ma et al., 2018). These arousal nuclei project into the cortex either directly or via the medial thalamus, and govern cortical states at both the global and local levels. Defined cortical regions have been shown to be remotely modulated, for instance, by inhibitory projections of the thalamic reticular nucleus (Lewis et al., 2015; Herrera et al., 2016; Fernandez et al., 2017) or excitatory projections of cholinergic neurons in a modality-selective manner (Kim et al., 2016; Záborszky et al., 2018).

Sensory stimuli are processed differently against the background of diverse cortical states (Shimaoka et al., 2018; Poulet and Crochet, 2019). Ensembles of neighboring neurons depend on a certain level of desynchronization to encode complex features of stimuli by concerting the firing rate, spike timing, and the temporal order at which they fire (Hopfield, 1995; Montemurro et al., 2008; Kayser et al., 2009; Mohajerani et al., 2013; Luczak et al., 2015; Montijn et al., 2016). Increasing synchronization may lend structure to features, but can also obscure them (Fries et al., 2007; Pachitariu et al., 2015; Olcese et al., 2018). An intermediate level of arousal has been found to enhance the consistency and signal strength of encoded stimuli and is associated with optimal sensory processing (Polack et al., 2013; Schneider and Logan, 2014; Schölvinck et al., 2015; McGinley et al., 2015a; Olcese et al., 2018; Shimaoka et al., 2018). Cortical information processing is substantially shaped by the activity of diverse inhibitory interneurons and relies on a balanced interplay of excitatory and inhibitory inputs (Isaacson and Scanziani, 2011; Rubin et al., 2017). Cortical states can become altered regionally and globally upon "attention" to a stimulus (Olcese et al., 2018; Poulet and Crochet, 2019). This flexibility permits the mode of sensory processing to adapt to situational demands.

Such modes of attention, sensory processing, and integration have been described in terms of cortical oscillations of distinct wavelengths (delta, theta, alpha, beta, and gamma, as reviewed in Fries et al., 2007; Schroeder and Lakatos, 2009; Saalmann et al., 2012; Fries, 2015; McVea et al., 2016; Jensen et al., 2019; Sikkens et al., 2019). The underlying rhythmic synchronization of

\section{A wakefulness

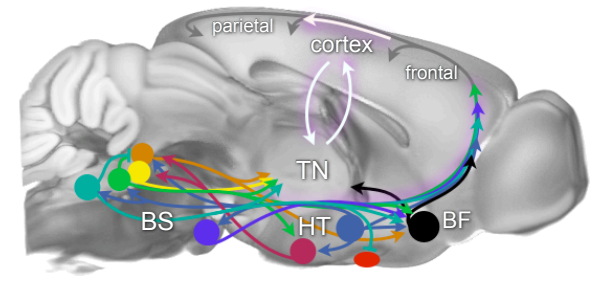 \\ B GABA $_{A}$ agonists

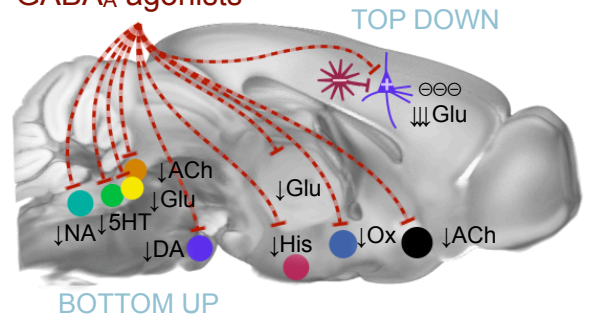 \\ anatomical regions \\ TN thalamic nuclei HT hypothalamus \\ BF basal forebrain BS brainstem \\ C a2 adrenoreceptor

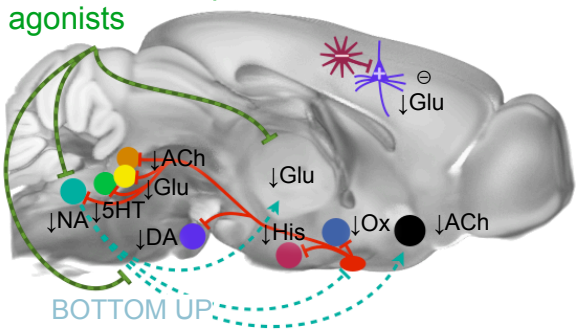 \\ D NMDA antagonists

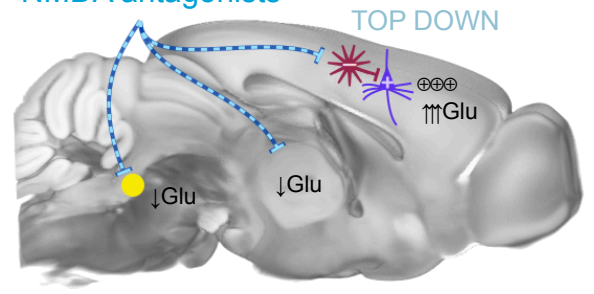 \\ - locus ceruleus \\ - basal forebrain \\ - preoptic area \\ - tuberomamillary nucleus \\ - ventral periaqueductal gray / ventral tegmental area \\ - perifornical hypothalmus \\ - raphe nucleus \\ parabrachial nucleus \\ - pedunculopontine / lateral dorsal tegmentum}

FIGURE 1 | Principal mechanisms of anesthesia. (A) Brain states are governed by a highly interconnected assembly of subcortical (arousal) nuclei. These nuclei employ distinct transmitter systems, including glutamate (Glu), noradrenaline (NA), serotonin (5HT), dopamine (DA), acetylcholine (ACh), histamine (His), and orexin (Ox). They project to the cortex directly and via higher-order nuclei of the thalamus. Thalamus and cortex are densely interconnected and heavily exchange information. Synchronous rhythmic thalamocortical activity can set the phase relations of distant cortical areas. Similar phase relations facilitate information transfer across the cortex and from frontal to parietal regions. All anesthetics generate distinct thalamocortical rhythms and alter the phase relationship of transmitting and receiving areas, leading to a successive breakdown of cortico-(thalamo-)cortical communication and eventually loss of consciousness. (B) GABA $\mathrm{A}_{\mathrm{A}}$ agonists, including volatile ethers, affect brain states both top-down and bottom-up by inhibiting excitatory neurons in subcortical nuclei and directly in the cortex. (C) $\alpha 2 A R$ agonists exert their effects bottom-up mainly by inhibiting the locus coeruleus, which leads to a disinhibition of sleep promoting neurons in the preoptic area of the hypothalamus. (D) N-methyl-d-aspartate (NMDA) antagonists act primarily top-down in a dual mode: at low doses by inhibiting inhibitory interneurons leading to cortical excitation, and at higher dosages by also inhibiting excitatory cortical pyramidal neurons. Further suppression of nociception and arousal is mediated by blocking the parabrachial nucleus. These three routes present the major principles of anesthesia. All anesthetics in current use in preclinical fMRI act in one of these ways. Inspired by Franks (2008), Lee and Dan (2012) and Akeju and Brown (2017), plotted on an MR reference template of the Allen mouse brain atlas (Bakker et al., 2015). 
neural assemblies is largely produced by a synchronized spiking of inhibitory interneurons at higher frequencies (reviewed in Fries et al., 2007; Jensen et al., 2019). The high magnitudes of slower rhythms are generated by other mechanisms (see "Brain States Under Anesthesia" section; reviewed in Neske, 2015; Sanchez-Vives et al., 2017). Neurons that act in phase are more likely to fire together, since the active and refractory periods of presynaptic and postsynaptic neurons are aligned (Saalmann, 2014). This temporal coding scheme can facilitate or inhibit information transfer between local and distant cell groups by varying their phase relations.

Cortical oscillations can propagate as traveling waves of neural depolarization and spiking activity across the cortical surface (reviewed in McVea et al., 2016; Kuroki et al., 2018; Muller et al., 2018). Higher frequencies are thereby nested within slower waves, leading to rich patterns of background information onto which local events are processed. The distinct and complex spatiotemporal dynamics of these traveling waves (e.g., radial, planar, spiraling, or rotating) are coordinated by recurrent cortical networks and distant projections from thalamic and subthalamic nuclei (Bhattacharya et al., 2019). Anesthesia can affect neural information processing at both the local level (by setting an imbalance of inhibitory and excitatory inputs) and globally (by spoiling phase relations of cell ensembles and disrupting information transfer across brain areas).

\section{Brain States Under Anesthesia}

Selecting an appropriate anesthetic protocol is in first place a question of choosing the right anesthetic class and dosage. Anesthetics currently used for murine fMRI (Table 1) can be divided into three classes based on their main molecular target receptors: $\gamma$-aminobutyric acid subtype A $\left(\mathrm{GABA}_{\mathrm{A}}\right)$ receptor agonists including volatile ethers, $\alpha 2$ adrenoreceptor ( $\alpha 2 \mathrm{AR})$ agonists, and $N$-methyl-d-aspartate (NMDA) receptor antagonists. Each class exerts its sedating effects based on one of three key principles: (1) top-down by inhibiting cortical neurons directly (NMDA receptor antagonists); (2) bottom-up by suppressing subcortical arousal nuclei that affect cortical states ( $\alpha 2 \mathrm{AR}$ agonists); or (3) both, by inhibiting neurons across the entire brain $\left(\mathrm{GABA}_{\mathrm{A}}\right.$ agonists and volatile ethers; Figure 1).

Because their target sites differ, each anesthetic class produces distinct cortical oscillatory dynamics (Figures 2A,B; Ching and Brown, 2014; Purdon et al., 2015; Flores et al., 2017), which vary with the anesthetic dosage (Figures 2B,C). Most anesthetics within one class further exhibit variations in their cortical signatures as they act at multiple receptor types (Table 1), interfering with partially overlapping pathways. Scientific advances over the last decades have revealed an increasingly comprehensive, yet still incomplete picture of the underlying mechanisms (Steriade, 2000; Campagna et al., 2003; Alkire et al., 2008; Franks, 2008; Brown et al., 2010, 2018a; Akeju and Brown, 2017; Flores et al., 2017; Hemmings et al., 2019). Here, we briefly introduce current models on how these rhythms are generated by the effects of different anesthetics, and how the brain states that are indicated by distinct rhythms affect neural transmission and processing.

\section{Thalamocortical Rhythmogenesis}

The generation of cortical rhythms by anesthesia follows a common pattern for all anesthetics (as described in more detail below). All anesthetics exert their sedating effects by acting on specific target receptor types (Table 1), leading to inhibition and thus hyperpolarization of the affected neurons. Many types of excitatory and inhibitory neurons comprise specific membrane channels that open at distinct levels of hyperpolarization and lead to inward cation currents that cause the cells to quickly depolarize. Hence, the affected cells respond to strong inhibition with excitation, leading to post-inhibitory rebound spiking. These bursts of neural activity recur periodically, paced by the sum of inhibitory (hyperpolarizing) and excitatory (depolarizing) inputs that are required to exceed the threshold for rebound spiking. These inputs can be differently modulated by distinct anesthetic types and dosages. The resulting spiking patterns reverberate in recurrent neuronal circuits and can synchronize large networks of cortical neurons by reciprocal coupling, which manifests as cortical oscillations on EEG. These oscillations are generated either within the cortex or the thalamus, or between these two densely interconnected regions (Figure 3A; reviewed in McCormick and Bal, 1997; Steriade, 2001; Fuentealba and Steriade, 2005; Franks, 2008; Ching and Brown, 2014; Akeju and Brown, 2017).

Thalamic key players of rhythm generation are excitatory thalamocortical (TC) cells in intralaminar and medial thalamic nuclei (Baker et al., 2014; Flores et al., 2017). These higher-order TC nuclei receive and process input from cortical areas and serve as crucial relays of information transfer across the cortex (Theyel et al., 2010; Sherman, 2017; Mo and Sherman, 2019). Effective information transfer is thereby facilitated by frequency and phase synchronization of projection, relay, and target areas (Saalmann, 2014). In contrast, first-order TC nuclei primarily relay incoming sensory information to the cortex. First and higher-order TC nuclei undergo a major inhibitory impact of GABAergic thalamic reticular (RE) neurons, which project from the thin, outer shell of the thalamus - the thalamic reticular nucleus (as reviewed in Varela, 2014). RE neurons control TC traffic, synchronicity of firing patterns, and the level of arousal by targeted inhibition of TC cells (Sun et al., 2012). The level of depolarization in both TC and RE neurons is further regulated by afferents from cholinergic and monoaminergic arousal nuclei (Saper et al., 2010), which also modulate the membrane potential of cortical neurons: GABAergic inhibitory interneurons and glutamatergic excitatory pyramidal cells (Figure 3A).

\section{$\mathrm{GABA}_{\mathrm{A}}$ Agonists Suppress Neural Activity Across the Central Nervous System}

Volatile ethers and $\mathrm{GABA}_{\mathrm{A}}$ agonists such as isoflurane and propofol predominantly act on postsynaptic $\mathrm{GABA}_{\mathrm{A}}$ receptors of excitatory neurons by increasing the preference of those ligand-gated chloride channels for the open state (Bai et al., 1999; Lee and Maguire, 2014). This enhances the inward chloride current and facilitates hyperpolarization and thus 


\section{A awake}

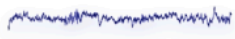

ү, $\alpha, \delta$ (low power)

propofol (GABAA)

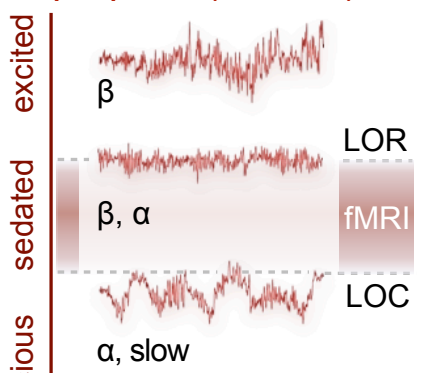

WWWWW Wh uP slow-ס

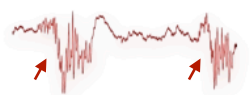

burst suppression

isoelectricity

dexmedetomidine ( $\alpha 2)$

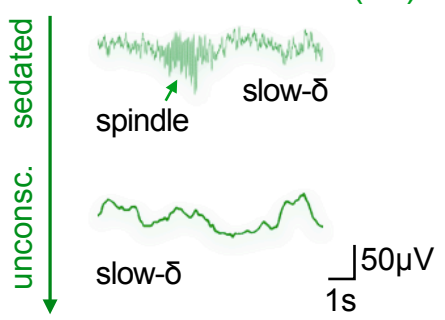

B
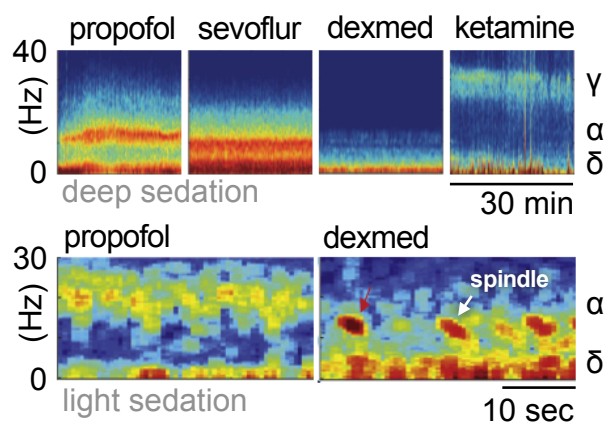

C
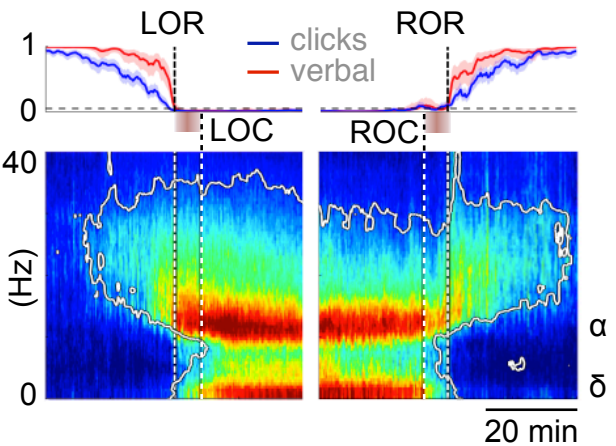

D

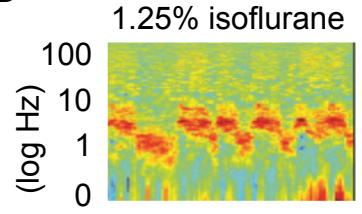

E

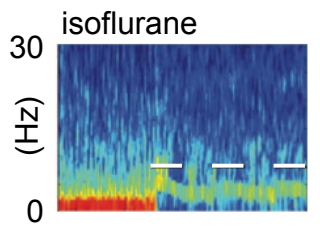

$1 \%$ isoflurane

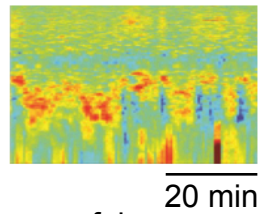

propofol

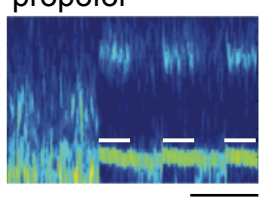

$\overline{1 \min }$

FIGURE 2 | Cortical oscillatory signatures of brain states under anesthesia. (A) Changes in oscillatory signatures for increasing doses of propofol and dexmedetomidine measured from the human anterior cortex. With increasing anesthetic depth, oscillation frequencies decrease and synchronize. (B) Distinct oscillatory signatures induced by different anesthetics (upper panel) and dosages (lower panel). The spectrogram represents the spectrum of frequencies in a time and frequency domain, which facilitates the identification of structured frequency bands. (C) Loss of responsiveness (LOR) and connected consciousness (LOC) induced by increasing doses of propofol. LOR is defined by suppression of responses to click and verbal commands (upper panel). Note the occurrence of alpha bands and the absence of slow-delta bands in the phase between LOR and LOC in the spectrogram. The same is true for return of consciousness (ROC) and responsiveness (ROR). In rodents, alpha waves coincide with the loss of righting reflex (LORR), which corresponds to LOR, and slow-delta waves are indicated by a complete loss of movement (LOM), which corresponds to LOC. (D) Brain states may not be fully stable when maintained via anesthesia. Transitions between two or more intermediate states occurred over longer periods of constant isoflurane concentration in rats. This feature has been defined as metastability. Note the logarithmic scale used to highlight the transitions that occur primarily in the lower frequency band $(0-10 \mathrm{~Hz})$. Local field potentials (LFP) recording was conducted in the anterior cingulate cortex (ACC). (E) Stimulation (white bars) of dopaminergic neurons of the ventral tegmental area in the rat shifts cortical states under sedation from slow delta $(<4 \mathrm{~Hz}$ ) towards $\theta$ power (isoflurane), or towards $\theta$ and $\beta$ power (propofol). Oscillations: slow $(<1 \mathrm{~Hz}), \delta(1-4 \mathrm{~Hz}), \theta(4-8 \mathrm{~Hz}), \alpha(8-15 \mathrm{~Hz}), \beta$ (15-30 Hz), awake- $\gamma(30-80 \mathrm{~Hz})$, ketamine- $\gamma(25-35 \mathrm{~Hz})$, spindle (9-16 Hz). Adapted with permission from (A,B) Purdon et al. (2015), (B, lower panel) Akeju and Brown (2017), (C) Purdon et al. (2013), (D) Hudson (2017), and (E) Solt et al. (2014).

inhibition of the postsynaptic cell. Presynaptic actions that impair neurotransmitter release (Hemmings et al., 2005, 2019; Baumgart et al., 2015) further contribute to reducing neural activity across the central nervous system (CNS), including the cortex, thalamus, striatum, brainstem, and even the spinal cord (Figure 1B; Bowery et al., 1987; Hemmings et al., 2005; Brown et al., 2010; Phillips et al., 2018). This exerts hypnotic effects and gradually renders the animal unconscious in a dose-dependent manner (Akeju and Brown, 2017; Flores et al., 2017).

An initial excitement is often observed at the induction of anesthesia, accompanied by a disinhibition of motor activity and the emergence of relatively fast cortical oscillatory activity in the 


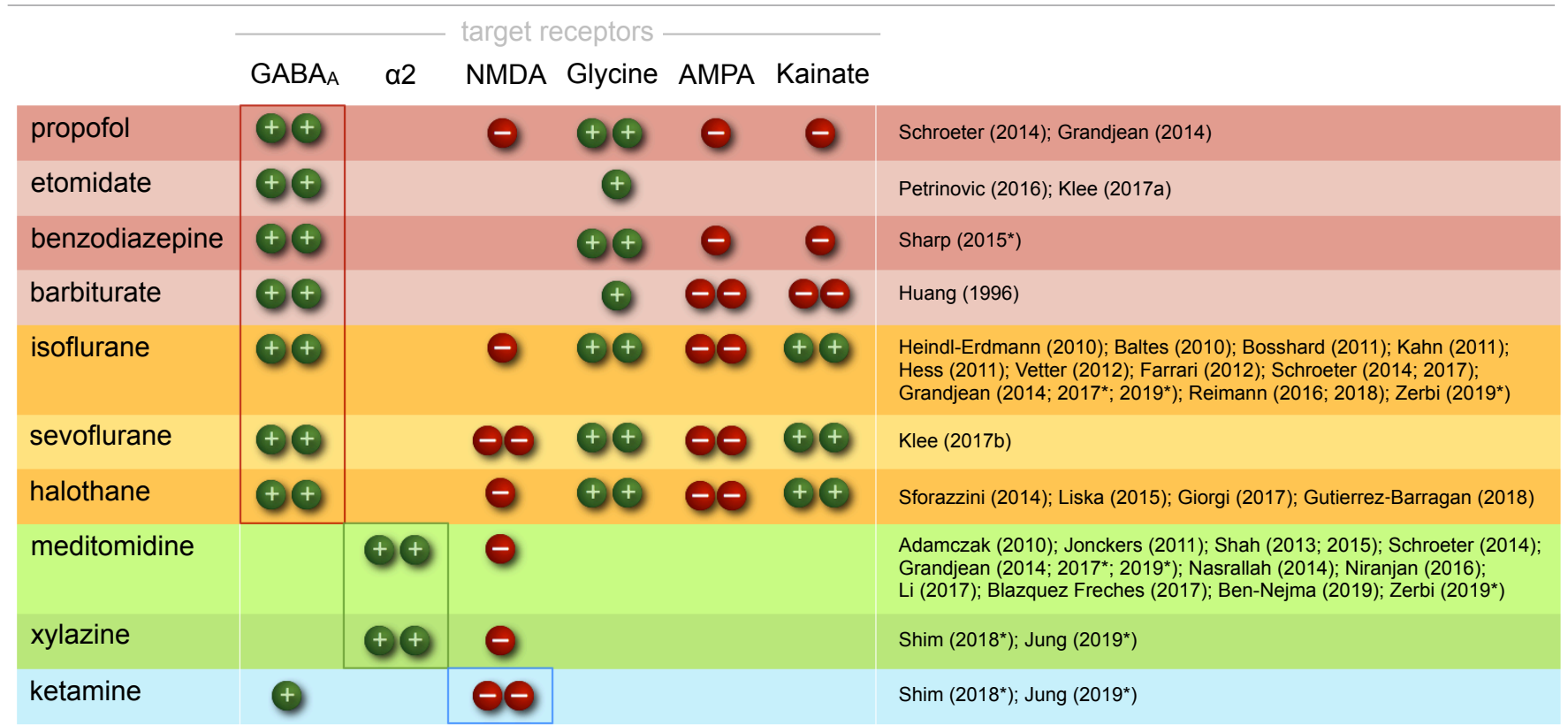

Each drug addresses distinct receptor types by either agonistic (+) or antagonistic (-) modulation. Single or double symbols are used to indicate whether potentiation or inhibition is mild or strong. Anesthetics are grouped into three classes based on their main target receptors: GABA agonists, including injectable drugs and volatile ethers (red and yellow, respectively), $\alpha 2 A R$ agonists (green), and NMDA antagonists (blue). Alongside these key target receptors, the listed drugs modulate a host of further receptor types and may contribute to specific anesthetic effects (see, e.g., Alkire et al., 2008; Franks, 2008; McKinstry-Wu and Kelz, 2019). Halothane is chemically not an ether, yet it belongs to the group of halogenated vapors, like isoflurane and sevoflurane, which share largely overlapping target sites. Note that only drugs suitable for longitudinal studies are listed; the GABA $A_{A}$ agonists $\alpha$-chloralose and urethane have also been applied in murine fMRI (Ahrens and Dubowitz, 2001; Xu et al., 2003, 2005; Grandjean et al., 2014; Schroeter et al., 2014) and will be discussed in "Sensory Processing and the Key Challenges in Murine fMRl" and "Functional Connectivity and Murine Resting-State fMRl" sections. References are shortened to first author (and year) to save space and allow for more comprehensive referencing. Asterisks indicate use in multimodal protocols.

beta band ( $\sim 13-30 \mathrm{~Hz}$; Figure 2A; Ching and Brown, 2014; Le Van Quyen et al., 2016). The precise mechanisms responsible for this paradoxical excitation have yet to be fully explained. Network models indicate the involvement of hyperpolarizationtriggered inward currents that cause cortical circuits of excitatory and inhibitory neurons to produce periodic firing in the beta range (McCarthy et al., 2008). With increasing inhibitory tone and sedation, beta rhythms slow down to alpha frequencies (Figures 2A, 3A; McCarthy et al., 2008; Baker et al., 2014; Flores et al., 2017). This is the onset of stable sedation marked by the loss of the righting reflex (LORR; Baker et al., 2014; Flores et al., 2017).

Although alpha oscillations $(8-15 \mathrm{~Hz})$ are produced within the cortex (Mukamel et al., 2014), the generation of temporally and spatially coherent alpha rhythms across larger areas requires the participation of the thalamus (Ching et al., 2010; Flores et al., 2017). The GABAergic inhibition of TC cells is thought to trigger enhanced post-inhibitory rebound spiking that is paced by the decay rate of inhibition, and thus depends on the anesthetic dosage (Figure 3A; Ching and Brown, 2014). Thalamic and cortical alpha activities reinforce each other by reciprocal coupling, resulting in strong TC synchronicity (Figure 3A; Ching et al., 2010; Ching and Brown, 2014; Crunelli et al., 2015; Bhattacharya et al., 2019).

Dense recurrent projections from the thalamus convey broad spatial coherence of alpha oscillations in the frontal cortex (Flores et al., 2017). In contrast, occipital thalamic projections of high-threshold TC neurons, which produce coherent alpha oscillations in the awake state are suppressed by a reduction of hyperpolarization-activated cation currents, presumably by secondary drug effects (Ching et al., 2010; Cimenser et al., 2011; Vijayan et al., 2013). This causes a characteristic spatial shift in EEG alpha power from the posterior to the anterior part of the brain, a phenomenon known as anteriorization (Akeju et al., 2014b).

LORR is likely caused by impaired information transfer and integration across cortical areas due to changing oscillatory modes. When sending and receiving cells are tuned in phase, postsynaptic neurons receive input during their active periods and are more likely to fire. Phase alignment facilitates neural transmission across distant cortical areas, which also involves higher-order TC nuclei (Saalmann, 2014; Sherman, 2017; Mo and Sherman, 2019). Higher-order TC nuclei are engaged with GABAergic drug-induced frontal alpha waves in humans and rodents (Ching et al., 2010; Liu et al., 2013a; Baker et al., 2014; Flores et al., 2017), which might affect phase-frequency relationships between sending and receiving areas and thus contribute to the disruption of cross-cortical communication.

However, the loss of awake-alpha (and higher frequency) waves has been shown to be a more reliable marker for stable sedation (Blain-Moraes et al., 2015; Pavone et al., 2017). A loss of awake-alpha is typical for the first stage of non-rapid eye movement (N1) sleep (Prerau et al., 2017) and was correlated 


\section{A thalamocortical circuit}

oscillations

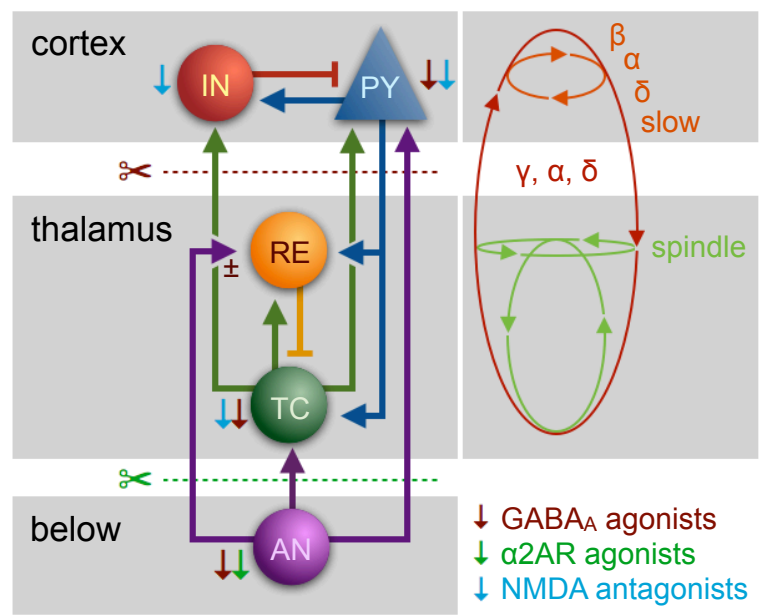

\section{B cortico-cortical information transfer}

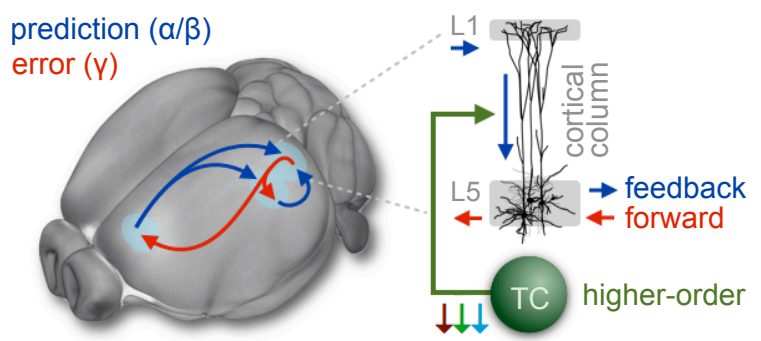

FIGURE 3 | The thalamocortical circuit as wave generating unit, and cortical information transfer. (A) Scheme of thalamocortical circuit displays major interactions between GABAergic inhibitory interneurons (IN) and excitatory pyramidal cells (PY) in the cortex and thalamic GABAergic inhibitory reticular cells (RE) and excitatory thalamocortical neurons (TC). Arousal nuclei (AN) drive cortical desynchronization via cortical and thalamocortical circuits, including biphasic excitatory-inhibitory modulation (Sun et al., 2013). Anesthetic classes reduce the membrane potential of their respective target neurons (indicated by thin color-coded arrows), leading to inhibition and rebound spiking. Cortical oscillations are produced by synchronized periodic activity between two or more cell types. Transection below cortex (red scissors) leads to slow wave generation in the cortex; transection below thalamus (cerveau isolé, green scissors) leads to generation of sleep-like spindles and slow-delta waves. Anesthesia-induced oscillations: slow (<1 Hz), $\delta(1-4 \mathrm{~Hz}), \alpha(8-15 \mathrm{~Hz}), \beta(15-30 \mathrm{~Hz}), \gamma(25-35 \mathrm{~Hz})$, spindle $(9-16 \mathrm{~Hz})$. (B) Fronto-parietal information transfer along the cortical hierarchy. Feedback information (blue) is conveyed from higher-order frontal areas to primary sensory areas in the $\alpha / \beta$ band, feedforward information flow (red) from primary to higher-order areas in the $\gamma$ band. In the predictive coding scheme feedback signals are considered predictive models of upcoming sensory states, whereas feedforward information represents the error resulting from a mismatch of a predictive model with an actual state. Feedback transmitted via long-range projections enters cortical columns at the dendritic trees of pyramidal cells in layer 1 (L1). The signal is transmitted to the soma of the same cells in layer 5, which requires driving input of higher-order TC nuclei. Feedback information transfer breaks down with the inhibition of higher-order TC nuclei at increasing depth of anesthesia. (A) Inspired by Ching and Brown (2014) and others. (B) Left panel: adapted from Sikkens et al. (2019). Note that visual higher-order areas in mice differ from those of primates, as reviewed in Glickfeld and Olsen (2017); Pennartz et al. (2019). Right panel: based on Suzuki and Larkum (2020). with a lack of behavioral response to stimuli in N1 sleep (Prerau et al., 2014) and under light sedation via sevoflurane (Pavone et al., 2017).

Awake-alpha waves play an essential role in information transfer and integration across the cortex (Jensen et al., 2019; Senzai et al., 2019). For instance, neural activity in the alpha band in contralateral sensorimotor areas has been shown to phase lock robustly to somatosensory stimuli. If these stimuli are perceived consciously, the stimulus locking spreads rapidly to the frontal, parietal, and ipsilateral sensorimotor regions (Palva et al., 2005). For unperceived stimuli, the phase locking is weak and restricted to the initial sensorimotor area.

Synchronous alpha activity in frontal, parietal, and sensorimotor regions facilitates recurrent information transfer between these areas due to a phase alignment of neural activity in transmitting and receiving areas. Such alpha-phase synchrony between primary sensory and higher-order cortical areas is considered a neural basis of attention, and is strongly mediated by frontal and parietal regions. Fronto-parietal information transfer is involved in many higher-order functions, including the formation of higher-order functional networks in the awake, resting state (see "Functional Connectivity and Murine Resting-State fMRI” section; Coull, 1998; Kastner and Ungerleider, 2000; Rees et al., 2002; Zeman, 2004; Klimesch et al., 2007; Palva and Palva, 2007; Sadaghiani et al., 2012; Fries, 2015; Han et al., 2019; Sikkens et al., 2019).

Recurrent processing along the cortical hierarchy accounts for the contextual modulation of stimuli, including perceptual and semantic interpretation, endowing them with spatiotemporal context and behavioral significance (Pennartz et al., 2019). One function of recurrent processing is expressed in the predictive coding framework, in which the brain is constantly generating and updating sensory models of the world (as reviewed in Pennartz et al., 2019; Sikkens et al., 2019). Feedback projections from higher-order (e.g., prefrontal) to early sensory areas are thought to convey or facilitate predictive models of upcoming sensory states via alpha/beta oscillations. A mismatch of a predictive model with actual sensory input results in error signals that are presumably fed forward from early sensory to higherorder areas via gamma oscillations-through different, partially overlapping cortical layers-to update the model in a perpetual feedback/feedforward loop (Figure 3B, left panel; van Kerkoerle et al., 2014; Zhang et al., 2014; Bastos et al., 2015; D’Souza et al., 2016; Chao et al., 2018; Michalareas et al., 2016; Kissinger et al., 2018; Zhang et al., 2019).

Feedback and feedforward information transfer are considered cornerstones of effective signal processing in the conscious brain (as reviewed in Lamme, 2018; Pennartz et al., 2019). LORR can probably be attributed to impaired feedback or feedforward information transfer (Pavone et al., 2017; Sanders et al., 2018; Redinbaugh et al., 2020). Long-range feedback modulation, which in primary sensory areas promotes both alpha waves (Jensen et al., 2019; Sikkens et al., 2019) and desynchronized activity (Harris and Thiele, 2011; Ecker et al., 2016), undergoes a breakdown with increasing anesthetic depth (Imas et al., 2005b; Raz et al., 2014; Hentschke et al., 2017; Sanders et al., 2018; Murphy et al., 2019). 
A recent cellular study revealed that cortico-cortical feedback flow through the cortical layers (mediated by L5 pyramidal cells) requires the driving input of higher-order TC nuclei (Figure 3B, right panel; Suzuki and Larkum, 2020). Since these higher-order TC nuclei in turn depend on the driving input of cholinergic projections from the brainstem (Figure 3A; Trageser et al., 2006; Masri et al., 2006, 2008), they appear to be particularly susceptible to the effects of various anesthetics. At already $1 \%$ isoflurane the inhibition of higher-order TC nuclei causes a disruption of cortico-cortical feedback loops.

This causal relationship unifies two central hypotheses of consciousness, which had previously assumed a dependence on either cortical feedback or higher-order TC loops (Alkire et al., 2008; Boly et al., 2011; Mashour, 2014). Also, feedforward signal propagation toward higher-order cortical regions has been reported to be increasingly suppressed with thalamocortical inhibition (Massimini et al., 2005; Sellers et al., 2015; Casarotto et al., 2016; Hentschke et al., 2017; Sanders et al., 2018; Redinbaugh et al., 2020). Therefore, studying sensory pathways within the cortex of anesthetized mice is often limited to brain areas that receive direct sensory thalamic or subcortical input.

Neural responses to sensory stimuli in primary cortices are largely preserved across sensory modalities (Lamme et al., 1998; Detsch et al., 1999; Pack et al., 2001; Imas et al., 2005a; Greenberg et al., 2008; Schumacher et al., 2011; Haider et al., 2013; Milenkovic et al., 2014; Raz et al., 2014; Sellers et al., 2015). Yet, even low anesthetic dosages alter the spatial and temporal structure of neural firing patterns, thereby disrupting information processing, which relies on precise timing of ensemble activity (Luczak et al., 2015; Yuste, 2015). For example, neural responses to visual stimuli in mice anesthetized with $0.25-1 \%$ isoflurane extend into larger $\mathrm{V} 1$ areas and are temporally prolonged, as they are less shaped by directed inhibition compared to the awake state (Haider et al., 2013; Sellers et al., 2015). This has also been observed for other drugs and sensory modalities (Devonshire et al., 2010).

\section{Crossing the Borders: GABAergic Slow Waves and Burst Suppression}

Increasing doses of anesthetics bring a complete loss of movement (LOM), which coincides with a sudden rise in slow-delta power; i.e., delta oscillations (1-4 Hz) primarily in the frontal areas, and slow waves $(<1 \mathrm{~Hz})$ across the entire cortex (Figure 2A; Steriade et al., 1993b,c,d; Flores et al., 2017; Chamadia et al., 2019). Slow-delta waves are characterized by alternations of persistent desynchronized network activity (depolarized "UP" states) and generalized neural silence (hyperpolarized "DOWN" states) of varying duration (Steriade et al., 1993b,c,d, 2001; Luczak et al., 2007). Slow-delta rhythms impose strong oscillatory dominance on the cortical firing patterns by nesting oscillations of higher frequencies in distinctive sequences (Steriade, 2001; Fuentealba and Steriade, 2005). Delta oscillations are grouped themselves by slow waves into larger sequences, although both waveforms share a very similar structure.

The exact phase-amplitude coupling of slower and faster waves is a function of the anesthetic depth (Chamadia et al.,
2019). With light sedation, alpha waves lie in the trough of slow-delta oscillations. With increasing anesthetic depth, they experience a shift in phase until they ride on top of the slow waves' peaks. This phase-amplitude syntax causes a functional decoupling of neural activity, and thus cortical communication to collapse in crucial networks, leading to deep anesthesia (see "Functional Connectivity and Murine Resting-State fMRI" section).

Cortical delta and slow waves persist following removal of the thalamus, although under such conditions, slow waves (at $\sim 0.3 \mathrm{~Hz}$ ) clearly dominate the EEG (Figure 3A, red scissors; Steriade et al., 1993c; Steriade, 2001). Slow waves even occur in small cortical pieces in vitro (e.g., in the primary visual cortex) and are robust to various experimental perturbations (as reviewed in Sanchez-Vives et al., 2017). Therefore, they have been proposed to represent the default activity pattern of cortical networks. The UP states bear striking resemblance to the desynchronized firing patterns of the awake state (Destexhe et al., 2007). The transition between the two microstates originates from activity-dependent adaptation, which accumulates during UP states, attenuates neural transmission, and eventually switches to the DOWN state. In this state of neural silence (or strongly reduced activity), the network recovers its excitability until it elicits the next sudden transition to the UP state, forced up and amplified by the firing rates of neighboring neurons (Compte et al., 2003; Destexhe and Contreras, 2006; Braun and Mattia, 2010; Mattia and Sanchez-Vives, 2012; Neske, 2015; Sanchez-Vives et al., 2017).

Delta oscillations consist of cortical and thalamic components that are generated in overlapping higher-order TC nuclei along with alpha oscillations at increasing inhibitory tone (Steriade et al., 1993c, 1994; Amzica and Steriade, 1997; Steriade, 2001; Flores et al., 2017). Thalamic delta rhythmogenesis results from the interplay between two distinct hyperpolarization-activated currents within TC neurons, whereas GABAergic RE cells provide the hyperpolarizing input under natural conditions (Figure 3A; McCormick and Pape, 1990; Soltesz et al., 1991; Steriade et al., 1994). Since TC cells are not synaptically coupled, they fire in frequency, but not necessarily in phase (Amzica and Steriade, 1997; Neske, 2015). Coupling of the clock-like thalamic delta patterns is mediated through interconnected cortical pyramidal cells, which generate bursts at similar intrinsic delta frequencies, thereby synchronizing cortical areas via recurrent TC projections (Amzica and Steriade, 1997; Neske, 2015). While delta power rises coherently in anterior areas, slow waves occur all over the cortex but not necessarily in phase, leading to cortical fragmentation at moderate doses and thus impaired information transmission (Lewis et al., 2012).

An essential component in generating slow and delta oscillations is the inhibition of subcortical arousal nuclei, which causes cortical and TC neurons to lose their depolarizing driving input (Figure 3A; Steriade et al., 1991, 1993a). An inhibition of key arousal nuclei is indeed sufficient to explain delta and slow wave generation in sleep (reviewed in Weber and Dan, 2016) and quiet wakefulness (Neske, 2015). However, the pattern characteristics of slow waves under natural conditions are distinct from those elicited by GABAergic anesthesia, which are 


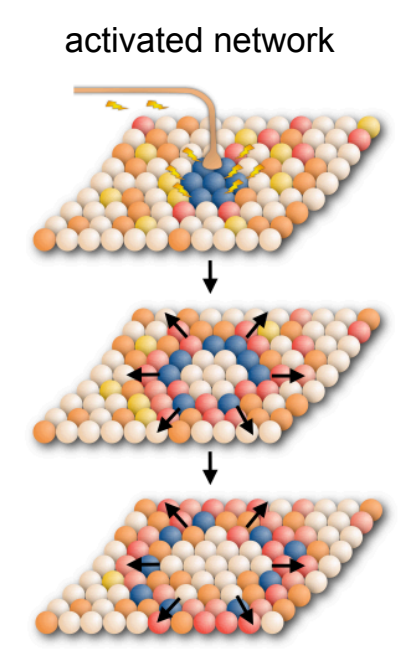

\section{quiescent network}

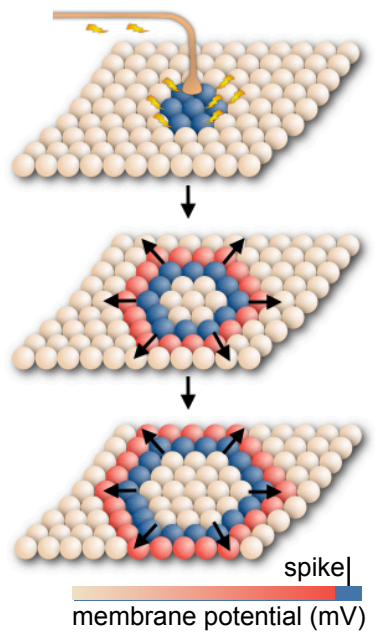

FIGURE 4 | Model for cortical signal propagation by nearest neighbor recruitment. In an activated cortical network with desynchronized background activity (as observed in normal waking states), local stimulation elicits waves that weakly entrain neuronal spiking as they travel across the network. In a quiescent cortical network with almost no background activity (as in deeply anesthetized states) local stimulation elicits dense traveling waves, which recruit nearly all cells as they pass. Spheres represent neurons whose membrane potential is indicated by color. Based on Muller et al. (2018).

produced under additional direct thalamic and cortical inhibition (Chauvette et al., 2011; Kenny et al., 2014; Busche et al., 2015; Akeju and Brown, 2017; Arena et al., 2017; Aggarwal et al., 2019). The full inhibitory package results in enhanced suppression of spontaneous desynchronized cortical background activity, which causes successively quiescent networks in the DOWN state with increasing anesthetic depth.

Such quiescent cortical networks of synchronous activity have been proposed to be ideal substrates for the propagation of dense traveling waves (Figure 4; Muller et al., 2018). Given that all neurons act in phase, neurotransmission reaches all post-synaptic cells during episodes of increased excitability. Triggering UP states in this low complexity environment instantly recruits neighboring neurons via massive recurrent excitation (Sanchez-Vives et al., 2017), so that even local events (like sensory inputs) can elicit dense traveling waves that entrain nearly all cells as they pass (Muller et al., 2018).

Such dense, traveling slow waves have been found to spread across the cortical surface, irrespective of anatomical boundaries, like a set of waves created by a drop in an oil bath (Figure 4; Massimini et al., 2004, 2007; Luczak et al., 2007; Stroh et al., 2013; Sanchez-Vives et al., 2017; Muller et al., 2018). Whether these waves remain local or propagate depends on the (local) brain state or desynchronization (Massimini et al., 2004, 2007; Nir et al., 2011; Vyazovskiy et al., 2011; Schwalm et al., 2017; Muller et al., 2018) and hence on anesthetic depth.

Aside from functional deafferentation and cortical inhibition, which provides a powerful substrate for long-range spread by nearest-neighbor recruitment, the synchronizing drive from the thalamus may add to the manifestation of slow waves (Crunelli and Hughes, 2010; Sheroziya and Timofeev, 2014; Crunelli et al., 2015; Neske, 2015). For the sake of translatability, functional brain studies should be generally performed at lower dosages than those leading to the dominance of slow wave and delta power, which affects neuronal excitability and stimulus-response properties (see "Anesthetic Depth and Consciousness-the Virtue of Translational Neuroimaging" section; Steriade et al., 1993b,c; Petersen et al., 2003; McGinley et al., 2015b).

Higher anesthetic dosages lead to burst suppressioncharacterized by increasingly prolonged "DOWN" micro-states alternating with periods of brief bursts of spikes and waves (Figure 2A; Steriade et al., 1994; Lewis et al., 2013; Amzica, 2015; Purdon et al., 2015). This is an increasingly hyperexcitable state in which large networks of cortical neurons suddenly discharge in tight synchrony (Steriade et al., 1994; Kroeger and Amzica, 2007; Ferron et al., 2009). Subsequently they fall into a post-burst refractory period, due to metabolic exhaustion (Hirsch and Taylor, 2010; Ching et al., 2012; Ching and Brown, 2014). This period of complete cortical silence is induced by adenosine triphosphate (ATP)-sensitive cation channels, which open when ATP decreases below critical levels (Cunningham et al., 2006). The resulting cation influx renders neurons unable to produce action potentials. Upon metabolic regeneration, the channels close again, and cortical networks gradually regain hyperexcitability by depolarizing, until they exhibit the next discharge (Kroeger and Amzica, 2007). Hyperexcitability is generated within the cortex as a result of the functional impairment of cortical afferents (Echlin et al., 1952; Henry and Scoville, 1952; Hughes, 1986; Niedermeyer et al., 1999; Kenny et al., 2014; Amzica, 2015).

Thalamic sensory transmission is preserved during isoelectric and burst episodes, although cortical discharge rates are substantially reduced during isoelectric periods (Detsch et al., 2002). During periods of cortical hyperexcitability, even subliminal stimuli are sufficient to trigger bursting activity (YliHankala et al., 1993; Hartikainen et al., 1995; Hudetz and Imas, 2007; Kroeger and Amzica, 2007; Amzica, 2009). Spontaneous and evoked bursts can spread across large cortical areas (Steriade et al., 1993b; Timofeev et al., 2000; Land et al., 2012) and may further engage remote hippocampal (Sirota et al., 2003; Hahn et al., 2006; Ji and Wilson, 2007; Busche et al., 2015) and thalamic structures (Steriade et al., 1993c; Stroh et al., 2013; Sheroziya and Timofeev, 2015), presumably by excitatory projections (Leong et al., 2016). In this regard, burst suppression events can be considered as non- or quasi-periodic slow waves with a long refractory time and high discharge.

Further increasing the anesthetic dosage leads to longer suppression, shorter burst periods, and less reactivity to stimuli (Hartikainen et al., 1995), which culminates in complete neural inexcitability and finally isoelectricity due to increasing (thalamo)cortical and subcortical suppression (Figure 2A; Kroeger and Amzica, 2007).

\section{$\alpha 2 A R$ Agonists Induce a Sleep-Like State by Suppressing General Arousal}

Sympatholytics or $\alpha 2 \mathrm{AR}$ agonists such as xylazine, medetomidine, or its potent dextro enantiomer 
dexmedetomidine exert their sedating and antinociceptive effects primarily by acting on presynaptic $\alpha 2_{\mathrm{A}}$ adrenergic receptors of noradrenergic cells that project from the locus coeruleus. This results in a hyperpolarization of the affected neurons and a reduction in the release of noradrenaline to their target sites (Correa-Sales et al., 1992; Jorm and Stamford, 1993; Chiu et al., 1995; Van Bockstaele et al., 1999). The locus coeruleus is a major arousal nucleus that projects to the basal forebrain (a subcortical arousal structure, which regulates cortical states by cholinergic efferents; Nelson et al., 2005; Hoover and Vertes, 2007; Pal et al., 2018), intralaminar nucleus of the thalamus, thalamic reticular nucleus, preoptic area of the hypothalamus, and diffusely into the cortex (Figure 1C; Asanuma, 1992; Nelson et al., 2003; Samuels and Szabadi, 2008a; Saper et al., 2010; Zhang et al., 2015; Fu et al., 2017; Brown et al., 2018a). Nociceptive pathways are further affected by the direct activation of $\alpha 2$ receptors in the spinal cord (Andrieu et al., 2009).

Decreased noradrenaline release in the preoptic area of the hypothalamus causes disinhibition (and thus excitation) of local endogenous sleep-promoting cells that send inhibitory projections to other key arousal nuclei in the midbrain and pons (Figure 1C; Sherin et al., 1998; Saper et al., 2005; reviewed in Saper et al., 2010; Scammell et al., 2017). This causes an inhibition of widely-projecting neurons in these arousal nuclei, which in turn decreases the depolarizing input to thalamic and cortical areas, leading to sleep-like, spindle $(9-16 \mathrm{~Hz})$, and slow-delta $(0.1-4 \mathrm{~Hz})$ oscillations. This pattern is distinct from that induced by $\mathrm{GABA}_{\mathrm{A}}$ agonists due to the lack of direct cortical and thalamic inhibition (Figures 2A-C, 3A; Noreika et al., 2011; Baker et al., 2014; Nasrallah et al., 2014a; Akeju and Brown, 2017; Banks et al., 2017).

Similar sleep-like states were observed following a transection below the thalamus in cats, the so-called cerveau isole (the isolated cerebrum; Figure 3A, green scissors; Bremer, 1935; Steriade et al., 1993c). This dramatically demonstrates that the disconnection of thalamic and cortical circuits from the input of subcortical arousal nuclei suffices to produce oscillatory and spiking patterns, similar to non-REM sleep at the $\mathrm{N} 2$ stage and sedation elicited by $\alpha 2 \mathrm{AR}$ agonists (Akeju and Brown, 2017).

Under light sedation, spindle rhythms occur in brief bursts of 0.5-3 s (Figures 2A,C; Baker et al., 2014; Nasrallah et al., 2014a; Purdon et al., 2015). Spindles are generated within the thalamic $\mathrm{RE}$ nucleus, which is considered the spindle pacemaker (Steriade et al., 1985; Halassa et al., 2011; Kim et al., 2012). Evidence for this includes the deafferentation of this structure from the cortex and the remaining thalamus, upon which the nucleus continues to generate spindles (Steriade et al., 1987). Rhythmogenesis thereby strongly depends on the level of hyperpolarization of thalamic RE neurons and the intactness of their long and thin dendrites, which are richly endowed with low-threshold hyperpolarizationtriggered $\mathrm{Ca}^{2+}$ channels (as reviewed in Fuentealba and Steriade, 2005; Crandall et al., 2010; Astori et al., 2011; Zaman et al., 2011).

Suppression of arousal nuclei leads to decreased depolarizing input to RE cells (McCormick, 1992; Saper et al., 2010; Sun et al., 2013), which can cause hyperpolarization below the threshold, leading to strong dendritic $\mathrm{Ca}^{2+}$ spikes. This results in depolarization and rhythmic bursts of action potentials that are sustained by additional voltage-gated cation channels (reviewed in Fuentealba and Steriade, 2005; Lüthi, 2014). GABAergic transmission and electrical coupling in recurrent networks of RE neurons are sufficient to synchronize oscillations in the range of spindles (Bazhenov et al., 1999; Fuentealba and Steriade, 2005).

In the intact brain, the initiation and synchronization of spindles is supported by TC and cortical circuits. Glutamatergic stimuli can easily trigger low-threshold $\mathrm{Ca}^{2+}$ spikes in $\mathrm{RE}$ cells (Crandall et al., 2010). Therefore, spindles can be initiated by diverse inputs, including spontaneous oscillating TC cells or cortical volleys that impinge on RE networks (Figure 3A; Destexhe et al., 1996). For example, the cortical transitions to the UP state of slow waves may be quickly followed by a resulting spindle wave, which is a common sequence in slow wave sleep, known as the K-complex (Steriade et al., 1993b; Amzica and Steriade, 1997). Notably, K-complexes have not been described for sedation through $\alpha 2 \mathrm{AR}$ agonists (Huupponen et al., 2008; Nasrallah et al., 2014a; Akeju et al., 2016a).

A burst of action potentials in an RE neuron causes hyperpolarization, rebound spiking and thus neural firing in multiple TC neurons; this reverberates in large thalamic networks by recurrent synapsing (Figure 3A; Bazhenov et al., 1999; Beenhakker and Huguenard, 2009). The strong synchronized rhythms of TC neurons can further entrain cortical pyramidal cells and interneurons in both prefrontal and sensory cortices (Peyrache et al., 2011). Cortico-cortical recruitment may cause further synchronization (Kandel and Buzsáki, 1997). The short spindle episodes are terminated via intrinsic ionic mechanisms in both thalamic RE and TC cells that are triggered by high concentrations of accumulated intracellular $\mathrm{Ca}^{2+}$ (alongside other strategies reviewed in Lüthi, 2014).

Delta waves resemble spindles in that they do not appear continuously in sleep or for light $\alpha 2 \mathrm{AR}$-induced sedation (Figure 2B; Steriade et al., 1993a,c; Baker et al., 2014). This changes at higher anesthetic dosages through a further suppression of depolarizing arousal inputs to thalamic RE neurons; they further hyperpolarize, and their firing patterns subsequently change from spindle to delta waveforms (Figures 2A,B; Nuñez et al., 1992; Destexhe et al., 1994).

The transition to delta rhythms is mirrored in TC circuits and entrains cortical networks. The result is continuous delta oscillation in thalamic and cortical areas, whose onset has been reported to coincide with a discrete drop in frequency at the instant of dexmedetomidine-induced LORR (Baker et al., 2014). This is accompanied by a significant phase shift of delta waves in the central medial thalamus as compared to cortical areas. Such phase shifts in higher-order TC nuclei can disrupt cortico-thalamo-cortical communication, causing a breakdown of information transfer between cortical areas (Slézia et al., 2011; Saalmann, 2014; Mo and Sherman, 2019; Suzuki and Larkum, 2020) and thus LORR.

First-order thalamic relay nuclei may not participate in producing continuous delta oscillations (Baker et al., 2014). Instead, they intensify spindle generation following LORR. The 
transient inhibition of TC neurons during spindle periods has been reported to prevent them from transferring sensory information to the cortex (Steriade and Contreras, 1995; Fuentealba and Steriade, 2005). Accordingly, spindle density correlates with the gating of sensory inputs in sleep (Dang-Vu et al., 2010; Wimmer et al., 2012; Chen et al., 2016).

However, in $\alpha 2 \mathrm{AR}$-induced sedation, primary sensory routes remain largely intact. Medetomidine has been shown to preserve cortical responsiveness to acoustic stimuli in primary sensory areas in rats (Banks et al., 2017). Subcutaneous electrostimulation of the paw has been reported to evoke slightly reduced potentials in the primary somatosensory cortex (S1) compared to GABAergic drugs (Hayton et al., 1999; Li et al., 2003). Yet, the amplitudes of somatosensory evoked potentials in S1 are not affected with increasing concentrations of medetomidine ( $\mathrm{Li}$ et al., 2003; Nasrallah et al., 2014a). Spindle activity in the paw region of S1 occurs at medetomidine concentrations that are commonly used for fMRI (Nasrallah et al., 2014a).

The induction of LORR at continuous delta activity has been demonstrated in a study that kept rats in a rotating tube in which they had to constantly adapt their position until they rolled onto their sides and remained supine (Baker et al., 2014). Such constant active behavior increases the activity of arousal nuclei (Marlinski et al., 2012; Furth et al., 2017) and interferes with the "sleep-like" sedative state elicited by $\alpha 2 \mathrm{AR}$ agonists (Kamibayashi and Maze, 2000; Venn and Grounds, 2001). In fact, sedation induced by $\alpha 2 \mathrm{AR}$ agonists is far more arousable than for GABAergic drugs (see "Sensory Processing and the Key Challenges in Murine fMRI" and "Functional Connectivity and Murine Resting-State fMRI" sections; Noreika et al., 2011; Sanders et al., 2012; Akeju and Brown, 2017; Banks et al., 2017). Thus, higher anesthetic dosages were required to induce LORR in actively moving rats (Baker et al., 2014) than to induce the sedation sufficient for an fMRI experiment (Nasrallah et al., 2014a). For the GABAergic drug propofol, LORR occurred with the onset of alpha waves both in rotating tubes (Baker et al., 2014) and in the freely resting rodent (Flores et al., 2017).

\section{NMDA Receptor Antagonists Primarily Affect Cortical Neural Activity}

Ketamine is an NMDA receptor antagonist that provides dissociating, quasi-hypnotic effects by selectively binding and blocking NMDA receptors that are primarily expressed in cortical inhibitory and excitatory neurons, but also in thalamic TC and RE cells (Deleuze and Huguenard, 2016), and to a lesser extent in subcortical arousal nuclei and peripheral nerves (Gunduz-Bruce, 2009). Ketamine generates opposing effects in a dose-dependent manner. At lower concentrations, it binds preferentially to NMDA receptors on cortical GABAergic inhibitory interneurons, which show about 10-fold higher sensitivity to NMDA blockade than pyramidal neurons (Grunze et al., 1996).

NMDA receptor blockage prevents cation influx and depolarization, resulting in decreased GABAergic transmission to downstream excitatory neurons (Homayoun and Moghaddam, 2007; Seamans, 2008). Thus, excitatory neurons are disinhibited and become depolarized (Figure 1D, Brown et al., 2011; Phillips et al., 2018; Picard et al., 2019). An appropriate depolarization of cortical pyramidal neurons can elicit fast oscillations in the lower gamma band (Steriade et al., 1991; Nuñez et al., 1992; Gray and McCormick, 1996), synchronized by rhythmic inhibition (Buzsáki and Chrobak, 1995; Olufsen et al., 2003; Börgers et al., 2005).

The fast rhythm generation is promoted by ketamine actions in thalamic circuits, which switches the firing patterns of TC and RE neurons from the burst mode to the tonic generation of single action potentials (Anderson et al., 2017; Mahdavi et al., 2019). Such synchronous fast oscillations in the gamma band $(25-80 \mathrm{~Hz})$ typically emerge with cortical processing during higher-level mental activity, and in REM sleep, which is associated with dreaming mentation (Llinás and Ribary, 1993).

Low-dose ketamine elicits gamma waves in a narrow frequency band of $\sim 25-35 \mathrm{~Hz}$ and diffuse excitatory cortical activity (Akeju et al., 2016b). At this stage, hallucinations, dissociated states, euphoria, and dysphoria have been reported in clinical use: they have been attributed to preserved communication across brain areas at low inhibitory modulation and control, as well as a disruption of dopaminergic neurotransmission in the prefrontal cortex (Moghaddam et al., 1997; Purdon et al., 2015).

At higher doses, ketamine increasingly begins to block NMDA receptors at excitatory pyramidal neurons, causing cortical inhibition to predominate. It further suppresses arousal pathways by blocking excitatory projections from the parabrachial nucleus and from the medial pontine reticular formation in the brainstem to the thalamus and to the basal forebrain (Boon and Milsom, 2008; Fuller et al., 2011; Brown et al., 2018a). In turn, gamma waves become interspersed with slow-delta oscillations (Figure 2B; Ruiz-Mejias et al., 2011; Akeju et al., 2016b), which are augmented by direct drug action in the thalamus (Kiss et al., 2011; Zhang et al., 2012). The suppression of higher-order TC loops (Suzuki and Larkum, 2020) and a breakdown of cortical coherence is considered likely to be the mechanism that induces LORR (Pal et al., 2015; de la Salle et al., 2016; Schroeder et al., 2016; Brown et al., 2018a).

Ketamine can cause regional hypo- and hyperactivation across the cortex (Porro et al., 2004) and introduces further complexity to the processing of external stimuli (Oye et al., 1992; Zandieh et al., 2003; Schwertner et al., 2018). Besides antinociception mediated by direct inhibition of peripheral nociceptive afferents expressing NMDA receptors (Sinner and Graf, 2008), ketamine has also been shown to persistently reduce aversive responses to noxious stimuli in a top-down manner by prolonged suppression of hyperactive neurons in the anterior cingulate cortex (ACC) in rodent chronic pain models (Zhou et al., 2018). Low dosages increase SEP in rats and mice, even compared to the awake state (Franceschini et al., 2010; Michelson and Kozai, 2018) although the degree depends on the mouse strain (Maxwell et al., 2006). SEP from the cortex to higher-order TC neurons are decreased, illustrating a disturbed functional state of cortico-thalamo-cortical and thus corticocortical circuits (Anderson et al., 2017). Low-dose ketamine increases power in the gamma band, but also delta power 
can increase more significantly than for low-dose isoflurane (Michelson and Kozai, 2018).

\section{Metastability of Brain States, Hysteresis, and Behavioral Monitoring}

The comparison of brain states induced by various anesthetic compounds and dosages illustrates the diversity of mechanisms that lead to sedation. Whether an adjusted brain state can be stably maintained throughout the entire duration of an fMRI session remains to be clarified. Volatile ethers and intravenously injectable anesthetics appear to permit relatively stable maintenance of cortical oscillation patterns (Figures 2B,C; Purdon et al., 2013, 2015; Flores et al., 2017). However, transitions between two or more brain states have been observed over longer periods $(1 \mathrm{~h})$ at fixed concentrations of isoflurane in rats (Hudson et al., 2014). The transitions occurred predominantly in the lower-frequency band and could be well observed when the data were expressed on a logarithmic scale (Figure 2D).

The authors referred to this condition as "metastable" or potentially "multistable," given that intermediate brain states shifted between two or more attractors distributed in phase space (for further discussion, see Breakspear, 2017; Hudson, 2017). There is evidence that such meta- or multistability is a general feature of brain states under anesthesia for various compounds and across species, although studies dedicated to detail such transitions over long periods are sparse (reviewed in Hudson, 2017). To what extent the transition of brain states may jeopardize the concordance of results across experiments (e.g., by exerting significantly different effects on signal processing) remains an open question, and has to be established for specific anesthetic protocols applied.

The reported state transitions were observed during recovery of consciousness; after an initial concentration of $1.75 \%$ isoflurane for $1 \mathrm{~h}$, which reliably produced burst suppression, the concentration was reduced by $0.5 \%$, maintained at that level for $1 \mathrm{~h}$, and the process was repeated over a total of $6 \mathrm{~h}$. Given this design, the dynamics of metastability were likely swayed by neural inertia-an intrinsic feature of neural circuits to resist swift transitions between consciousness and unconsciousness (Friedman et al., 2010; Proekt and Hudson, 2018; Proekt and Kelz, 2018). During recovery of consciousness neural inertia tends to trap the brain in an unconscious state. Due to this "stickiness," a lower anesthetic dosage is required to maintain a similar anesthetic depth for the emergence from unconsciousness, compared to the induction of anesthesia (Hudson et al., 2014; Hudson, 2017). This dependence of the brain state on its history (hysteresis effect) cannot be explained by pharmacokinetic actions (see "Functional Connectivity and Murine Resting-State fMRI" section; Kelz et al., 2008; Friedman et al., 2010), and should be considered in preclinical studies for which an initial bolus induction of anesthesia is common practice.

Another issue in maintaining an intermediate brain state throughout an fMRI experiment might arise from nociceptive or stressful stimuli. Clinical experience and preclinical studies have shown that noxious stimuli, as well as direct stimulation of key arousal nuclei, can shift cortical states from slow synchronized towards highly desynchronized oscillations (Figure 2E; Hudetz et al., 2003; Solt et al., 2014; Vazey and Aston-Jones, 2014; Akeju and Brown, 2017; Sanders, 2017; Pal et al., 2018; Hayat et al., 2019). When the activity of the arousal-related nuclei exceeds a certain level, the animal wakes up from light anesthesia, and falls back into sedation when the activity subsides (Figure 2E; Solt et al., 2014). Consequently, an anesthetic protocol should be tailored, in terms of class and dosage, to the experimental task at hand. If the aim of a study is to assess functional connectivity (FC) at rest, there is no need to adjust the anesthetic depth so that the mouse remains unresponsive to nociceptive stimuli.

This calls into question the idea of the minimum alveolar concentration (MAC) in rodent fMRI-a well-established behavioral measure of anesthetic depth for volatile ethers (Steffey, 2017). Briefly, a MAC of 1.0 is defined as the average of the lowest anesthetic concentration that prevents a behavioral response upon a standardized pain stimulus, and the highest concentration that still permits a nocifensive response in $50 \%$ of tested subjects (Eger et al., 1965; Quasha et al., 1980). A MAC of 0.7 in rats has been reported to suppress the righting reflex and active attempts to withdraw; at a MAC of 0.3 ("MAC-awake"), frequent movements of the snout including sniffing, chewing, licking, and gross limb movements still occurred (Hudetz, 2002). However, these behavioral markers are not dependent on the application of any nociceptive stimulus that is not applied in the actual experiment.

Anesthetic depth can be monitored by behavioral markers and pupil dilation diameter to assess the level of arousal (Erisken et al., 2014; McGinley et al., 2015a; Joshi et al., 2016; Reimer et al., 2016; Binda and Gamlin, 2017; Shimaoka et al., 2018). Behavioral observation is indispensable to determine the level of sedation and immobilization of an animal, although the capacity to infer the actual brain state from these responses is limited (Pal et al., 2018). Hence, it is highly encouraged to adjust and compare anesthetic protocols based on oscillatory signatures and neural response properties outside the MR environment. Behavioral monitoring becomes increasingly crucial as different anesthetic classes and combinations of drugs are employed to sedate the animal (see "Multimodal Anesthesia in Translational fMRI" section).

\section{Anesthetic Depth and Consciousness-the Virtue of Translational Neuroimaging}

In general, anesthetic mechanisms are highly conserved across species (Achermann and Borbély, 1997; Steriade et al., 2001; Mölle et al., 2002; Eschenko et al., 2006; Destexhe et al., 2007; Buzsáki and Moser, 2013; Shein-Idelson et al., 2016) and the oscillatory signatures of humans and mice are virtually similar upon applications of specific anesthesia and stages of anesthetic depth (Seth et al., 2005; Flores et al., 2017; Guidera et al., 2017; Hudson, 2017; Storm et al., 2017; Olcese et al., 2018). This relation provides a rough means with which to interpret brain states in terms of perceived anesthetic depth, stress, or pain. In humans, specific brain states can be linked to the introspective experience, as revealed by subjective reports. Using oscillatory signatures, a third-person observer can precisely specify the 
moment at which the "lights switch off," from the first-person perspective (Purdon et al., 2015; Brown et al., 2018b). For GABAergic drugs, this is the emergence of slow-delta oscillations (Figure 2C; Purdon et al., 2013, 2015).

Traditionally, this transition point is considered to be the "loss of connected consciousness" (LOC; Sanders et al., 2012) and is defined as a loss of sensory perception and interoception-the perception of internal processes, including pain, anxiety, stress, discomfort, or sense of time (Purdon et al., 2013, 2015; Warnaby et al., 2016; Sleigh et al., 2018; Chamadia et al., 2019). This is distinguished from "disconnected" phenomenal consciousness, like the awareness of pure darkness (Sanders et al., 2012; Sleigh et al., 2018) or dreaming, although dreams have often been reported for medetomidine-induced LOC (Akeju and Brown, 2017; Mashour and Hudetz, 2017, 2018).

In the state of propofol-induced LOC, innocuous sensory stimuli such as words or tones could no longer be perceived, and no hemodynamic responses to these stimuli were detected using fMRI (Ní Mhuircheartaigh et al., 2013; Warnaby et al., 2016; Lichtner et al., 2018). However, BOLD patterns in response to nociceptive stimuli were partially preserved (Lichtner et al., 2018). These findings imply that the oscillatory signatures associated with LOC may be useful as a marker delineating the lower limit of anesthetic depth in sensory fMRI (Figures 2A,C).

Another transition point can be determined based on behavioral observation-the "loss of behavioral responsiveness" (LOR), which occurs earlier, approximately with the appearance of frontal alpha oscillations and before slow waves dominate the EEG (Figure 2C; Purdon et al., 2013; Warnaby et al., 2016). At this point, subjects fail to exhibit volitional responses to sensory or even noxious stimuli (Sanders et al., 2012; Ní Mhuircheartaigh et al., 2013; Purdon et al., 2013; Warnaby et al., 2016; Sleigh et al., 2018). Stimuli are still perceived and processed when they are applied, as indicated by fMRI data and subjective reports (Warnaby et al., 2016). Volunteers described this as a state of detachment from the stimuli and from "themselves"-i.e., they were not fully unconscious, but also not aware that the stimuli were related to them. Tones that were presented rather subtly and very briefly $(1 \mathrm{kHz}$, $60 \mathrm{~ms}$ ) were not sufficient to elicit significant BOLD responses beyond the thalamic relay nuclei. The BOLD patterns evoked by words and nociception, on the other hand, were reported to reflect closely those of the conscious state, with substantially lower activity in only the right dorsal anterior insular cortex (Warnaby et al., 2016). This area is reportedly associated with body ownership and self-agency (Warnaby et al., 2016; Lichtner et al., 2018; Sleigh et al., 2018). The anesthetic inhibition of the anterior insula and related interoceptive networks led the authors to reformulate their understanding of the hypnotic effects of anesthesia as a gradual disruption of "selfhood," which occurs with an increase of anesthetic depth, finally leading to oblivion (Sleigh et al., 2018).

This offers not only a direct link between introspective reports, behavioral and neuroimaging markers, but also a vivid example of how the examination of large-scale networks using fMRI can complement models of LOC that are based on the suppression of feedback or feedforward information transfer along the cortical hierarchy (see "GABA A Agonists Suppress Neural Activity Across the Central Nervous System" section; reviewed in Pennartz et al., 2019; Sikkens et al., 2019). Because similar transitions in oscillatory signatures can be observed in humans and rodents, maintaining and stabilizing a brain state between LOR (corresponding to LORR in animals; Baker et al., 2014; Banks et al., 2017; Flores et al., 2017) and LOC (corresponding to LOM in animals, and the occurrence of slow-delta waves; Flores et al., 2017) could be an attractive target state in which to perform fMRI in mice, at least for sensory perception tasks (Figures 2A,C). Hemodynamic coupling does not appear to be directly affected by propofol (Veselis et al., 2005). However, potential vasomodulatory effects of anesthesia have to be taken into account when inferring dose-dependent suppression of neural activity based on fMRI (Table 2; Aksenov et al., 2015).

\section{MURINE IMRI AND HEMODYNAMIC INTEGRITY}

Research on murine fMRI has grown exponentially from only a handful of publications in the first decade (Huang et al., 1996; Ahrens and Dubowitz, 2001; Mueggler et al., 2003; Xu et al., 2003, 2005) to more than 50 in the following. Nevertheless, anesthesia in murine fMRI presents a multitude of challenges beyond the determination of functional brain states and neural response properties, and anesthetic protocols are still far from being effectively tailored to meet murine physiological and hemodynamic demands. Preserving hemodynamic integrity that approximates a physiological state is essential to exploit the two key advantages of murine fMRI: non-invasively investigating whole-brain functional dynamics, and the opportunity to directly compare these dynamics across species, including humans. The experiments that have been performed can be classified into at least four categories: (1) sensory perception; (2) nociception and pain; (3) FC based on the resting state; and (4) within-brain stimulation involving the use of opto- and chemogenetics. Each category reveals particular aspects of anesthetic effects in fMRI and deserves consideration in terms of the requirements for anesthetic protocols that are appropriate to obtaining neuroimaging data of translational value.

\section{Sensory Processing and the Key Challenges in Murine fMRI}

Sensory studies were among the earliest and most elementary applications of murine fMRI. Nevertheless, only a handful of publications report on innocuous sensory stimulation tasks that address the "natural" sensory perception routes of primary organs-like eyes, ears, nose, skin, or whiskers-using various anesthetic protocols. The very first mouse fMRI study (Huang et al., 1996) concerned visual perception and applied the $\mathrm{GABA}_{\mathrm{A}}$-positive allosteric modulator and agonist pentobarbital; subsequent olfactory studies used urethane (Xu et al., 2003, 2005). Later visual (Niranjan et al., 2016) and auditory 
(Blazquez Freches et al., 2018) studies applied the $\alpha 2 \mathrm{AR}$ agonist medetomidine, and deflection of the vibrissae was conducted under low-dose (0.5-1\%) isoflurane (Kahn et al., 2011). All these studies identified modality-relevant sensory pathways, including primary sensory and partially preserved secondary cortical and thalamic structures. Higher-order or association areas have not been reported.

\section{Technical Detection Limit}

While this is in principle an encouraging situation, the reality of implementing mouse fMRI in the laboratory is fraught with peril. Sensory fMRI requires meticulous fine-tuning of murine physiology to compensate for anesthetic side effects, in conjunction with advanced technical equipment to boost the BOLD effect, which increases linearly with the magnetic

\section{A}

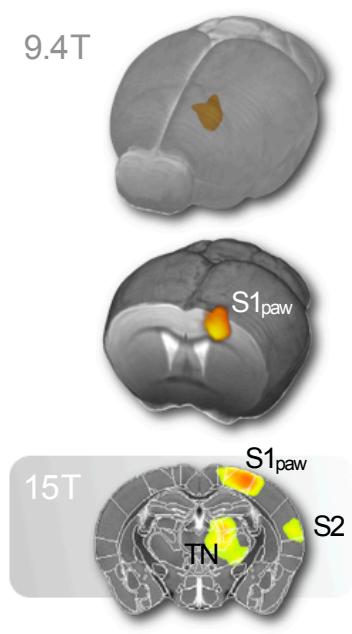

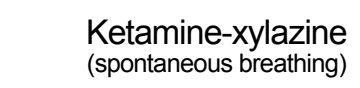

\section{$1 \%$ isoflurane (ventilated)}

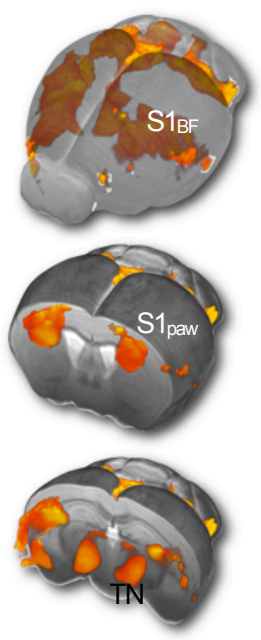

B

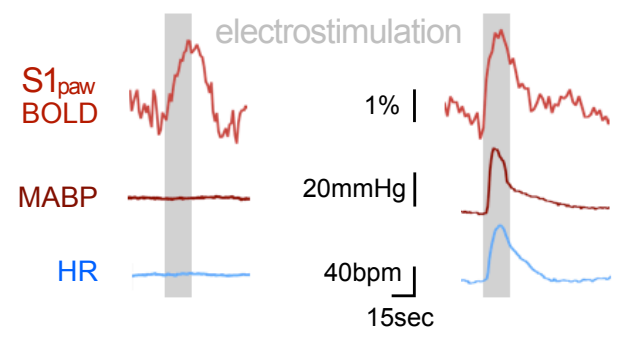

FIGURE 5 | Subcutaneous electrostimulation of the paw in spontaneous breathing and mechanically ventilated mice under two different anesthetic regimens. (A) Blood oxygenation level-dependent (BOLD) patterns show unilateral responses in the contralateral paw region of $\mathrm{S} 1$ for spontaneous breathing mice under ketamine-xylazine. Increasing the field strength to $15 \mathrm{~T}$ reveals additional pattern in S2 and thalamic regions not visible at $9.4 \mathrm{~T}$. In contrast large bilateral patterns were observed in ventilated mice under $1 \%$ isoflurane. (B) Signal time courses depict substantial surges in mean arterial blood pressure (MABP) and heart rate $(\mathrm{HR})$ in ventilated, but not in spontaneous breathing animals. Data were acquired in-house reproducing findings from Reimann et al. (2018) and Shim et al. (2018). Functional map superimposed on gray shading (15 T) is adapted with permission from Jung et al. (2019). field strength and venous blood volume (Kim, 2018). A recent study vividly illustrated how the field strength determines whether or not activation of entire brain areas may be detected. Subcutaneous electrostimulation of the murine paw is expected to elicit activity in the contralateral ventral thalamic nuclei, which relay the signal along the spinothalamic tract to the primary (S1) and secondary (S2) somatosensory cortices. Nevertheless, BOLD responses to electrostimulation were found to be limited to the contralateral S1 in mice under ketamine-xylazine at $9.4 \mathrm{~T}$ (Figure 5A; Shim et al., 2018), and under medetomidine at 9.4 (Nasrallah et al., 2014a) and $11.7 \mathrm{~T}$ (Adamczak et al., 2010). However, the same ketamine-xylazine protocol at $15 \mathrm{~T}$ revealed the expected BOLD patterns in all three somatosensory key areas (Figure $\mathbf{5 A}$; Jung et al., 2019).

To boost the BOLD signal, small-bore MR scanners can be equipped with a cryogenically cooled radiofrequency coil that amplifies the temporal SNR by a factor of up to 3 (reviewed in Niendorf et al., 2015). Increasing the statistical power by multiple repetitions of a task similarly facilitates the detection of weak BOLD effects. Both strategies reduce noise, but do not increase the sensitivity to $\mathrm{T}_{2^{*}}$-the measure of relative changes in blood oxygenation, which determines the intrinsic technical detection limit of an MR system. To amplify a signal, it must be above the detection threshold. However, weak BOLD responses have been observed in the S2 and the thalamus at 9.4 T (Jung et al., 2019), strongly suggesting that these areas can be detected via signal amplification. The studies reported above employed MR surface coils that lose sensitivity for deeper brain areas. In addition to this technical issue, the low BOLD signal in deeper brain areas is likely physiological in nature, and may be caused by the sparser overall thalamic cell density, compared to the cortex (Meyer et al., 2013), by different densities of noradrenergic afferents in these areas (King et al., 1995; Wang et al., 1996) or otherwise asymmetric impact of reduced subthalamic drive, by unequal anesthetic effects on the vasculature in different brain regions (Lei et al., 2001), or by the experimental setup that can create a thermal gradient between the dorsal-ventral axis of the mouse brain (see below).

\section{Vasomodulation, Body Temperature, and Respiratory Depression}

The modulation of vascular properties across the brain is a key challenge for fMRI under anesthesia-even more so due to the temperature sensitivity of vessels. All anesthetics affect thermoregulation and render mice poikilothermic. Due to their high surface area-to-mass ratio, mice quickly adopt to their environmental temperature. Although blood circulation distributes heat energy across the body, heating pads below the trunk and cool surface MR units attached to the scalp may induce thermal gradients across the mouse, which means the brain temperature is neither homogeneous across all areas, nor accurately reflected by the temperature reading of a rectal probe (Reimann et al., 2016). Aside from a thermal dependency of neural activity that has been shown to alter brain states, and hence anesthetic depth (Volgushev et al., 2000; Reig et al., 2010; Sheroziya and Timofeev, 2015; Schwalm and Easton, 2016), 
TABLE 2 | Effects on hemodynamic integrity of anesthetics used in mouse fMRI - hemodynamic effects

\section{$\mathrm{CBF} \quad \mathrm{CMRO}_{2} \quad$ ICP AutoReg $\mathrm{CO}_{2}$ Reg}

\begin{tabular}{l|ccccc}
\hline propofol & $\downarrow \downarrow$ & $\downarrow \downarrow$ & $\downarrow \downarrow$ & - & $\downarrow$ \\
\hline etomidate & $\downarrow \downarrow$ & $\downarrow \downarrow$ & $\downarrow \downarrow$ & - & $\downarrow$ \\
\hline benzodiazepine & $\downarrow$ & $\downarrow$ & $\downarrow$ & - & \\
\hline barbiturate & $\downarrow \downarrow$ & $\downarrow \downarrow$ & $\downarrow \downarrow$ & $-/ \downarrow$ & $\downarrow$ \\
isoflurane & $-\uparrow$ & $\downarrow \downarrow$ & $-\uparrow$ & $-/ \downarrow$ & $\uparrow$ \\
\hline sevoflurane & - & $\downarrow \downarrow$ & - & $\downarrow$ & $\uparrow$ \\
halothane & $\uparrow \uparrow$ & $\downarrow$ & $\uparrow \uparrow$ & - & $\uparrow$ \\
\hline meditomidine & $\downarrow$ & - & $\downarrow$ & - & - \\
xylazine & $\downarrow$ & - & $\downarrow$ & - & - \\
\hline ketamine & $\uparrow$ & - & $\uparrow$ & $\downarrow$ & \\
\hline
\end{tabular}

Anesthetics are listed according to their classes: $G A B A_{A}$ agonists, including injectable drugs (red) and volatile ethers (yellow), $\alpha 2 A R$ agonists (green), and NMDA antagonists (blue). Vascular effects including vasodilation and vasoconstriction may impact on cerebral blood flow (CBF), intracranial pressure (ICP), or cerebral autoregulation (AutoReg). Drugs may further affect the cerebral metabolic rate of oxygen consumption $\left(\mathrm{CMRO}_{2}\right)$ and the regulation of the $\mathrm{CO}_{2}$ metabolism $\left(\mathrm{CO}_{2}\right.$ Reg). Effect: $\downarrow$ reduction; $\downarrow \downarrow$ strong reduction; $\uparrow$ increase; $\uparrow \uparrow$ strong increase;-no change. Based on Bittner et al. (2019).

vessels dilate with increasing temperature. Vasoconstriction induced by cooling the scalp can boost cortical BOLD responses to electrostimulation by about $30 \%$, although this is not physiological (Baltes et al., 2011).

Many anesthetics directly affect vascular dilation and reactivity, which can substantially alter baseline $\mathrm{CBF}$ and hemodynamic responses (see Table 2, Franceschini et al., 2010; Masamoto and Kanno, 2012). In contrast to cooling, vasoconstrictive effects of $\alpha 2 \mathrm{AR}$ such as medetomidine and xylazine (Sinclair et al., 2003; Fukuda et al., 2013) might hinder the thorough vasodilation of arterioles in response to neural activity and impair BOLD responses in mice (Nasrallah et al., 2014a; Schroeter et al., 2014). Anesthetic-induced vasodilation, on the other hand, increases the baseline blood flow and hence decreases the magnitude of relative changes in $\mathrm{CBV}, \mathrm{CBF}$, and BOLD in response to neural activity. Isoflurane is commonly reported to have strong vasodilatory effects (Sharp et al., 2015; Petrinovic et al., 2016; Cao et al., 2017), causing decreases of up to $75 \%$ in $\mathrm{CBF}$ responses to whisker stimulation at a concentration of only $1 \%$ in spontaneously breathing mice (Takuwa et al., 2012). Although isoflurane has been shown to directly dilate cerebral vessels (Iida et al., 1998; Leoni et al., 2011), its major vasodilatory potency is a result of its suppressive effects on respiration in a dose-dependent manner (van Alst et al., 2019).

Respiratory depression leads to hypercapnia-elevated blood $\mathrm{CO}_{2}$ levels-which causes strong cerebral vasodilation and increases the baseline CBF (Kety and Schmidt, 1948; Raper and Levasseur, 1971; Shimosegawa et al., 1995; Jones et al., 2005; Leoni et al., 2011; van Alst et al., 2019). Both rats and mice exhibit massively reduced or even fully ablated BOLD responses, when $\mathrm{CO}_{2}(5-10 \%)$ is added to the inspiratory gas (Schlegel, 2017; Munting et al., 2019; van Alst et al., 2019). Mechanically ventilated rats showed substantially higher BOLD responses to subcutaneous electrostimulation, and lower baseline CBF compared to spontaneously breathing rats at the same concentrations of isoflurane (van Alst et al., 2019). A comparison of cortical and thalamic baseline perfusion across anesthetic protocols in mechanically ventilated mice found isoflurane to be in a similar range as urethane, which is known to preserve hemodynamics and vascular diameters (Schroeter et al., 2014).

Hypercapnia has further effects on brain states. Since $\mathrm{CO}_{2}$ forms carbonic acid in water, elevated $\mathrm{CO}_{2}$ makes the blood more acidic. Both low $\mathrm{pH}$ and $\mathrm{CO}_{2}$ have been shown to impair neural transmission and excitability (Coulter et al., 1995; Tombaugh and Somjen, 1996; Sun et al., 1997; Meuth et al., 2003, 2006; Putnam et al., 2004; Williams et al., 2007; Sinning and Hübner, 2013). Hyperoxia, on the other hand, has been reported to cause vasoconstriction of arterioles (Duling and Berne, 1970; Pries et al., 1995) and to decrease baseline CBF (Matsuura et al., 2001; Matsuura and Kanno, 2002). This demonstrates that blood gas levels are crucial in fMRI and that respiration should be kept at physiological levels to preserve neural and especially hemodynamic responses.

\section{Mechanical Ventilation and Stress Response}

This has led to the widespread use of mechanical ventilation in murine fMRI, initially to detect highly reproducible BOLD responses to salient ("attention grabbing") stimuli, like subcutaneous electrostimulation (Bosshard et al., 2010; Baltes et al., 2011), and later nociceptive heat stimuli (see "Nociception, Pain, and Physiological Confounds" section; Bosshard et al., 2015; Reimann et al., 2016). Mechanical ventilation was further introduced as part of a robust protocol for pharmacological fMRI in mice (Ferrari et al., 2012) and is utilized in resting-state fMRI (see "Functional Connectivity and Murine Resting-State fMRI" section; Grandjean et al., 2014). The commonly applied protocol features $1.0-1.3 \%$ isoflurane in combination with neuromuscular blockage to minimize motion-related noise and prevent stimulus-correlated movements. An application of classical electrostimulation block paradigms to the murine paw-stimulus trains of 15-30 s composed of rectangular pulses of $0.5-2 \mathrm{~mA}$-revealed that this protocol leads to BOLD signal changes of substantial magnitudes, and a very specific pattern of distribution: BOLD effects occurred in bilateral clusters in S1, S2, the thalamus, and the insular cortex (Figure 5A; Bosshard et al., 2010; Baltes et al., 2011; Schroeter et al., 2017; Reimann et al., 2018; Schlegel et al., 2018; Shim et al., 2018), and were accompanied by strong, transient elevations in mean arterial blood pressure (MABP) and heart rate (HR; Figure 5B; Schroeter et al., 2014; Reimann et al., 2018). This was confirmed for various anesthetic protocols including low-dose isoflurane, medetomidine, propofol, and urethane in ventilated and paralyzed mice (Schroeter et al., 2014).

The same stimulation paradigm applied to spontaneously breathing mice evoked less prominent BOLD responses that were strictly limited to the paw region of the contralateral S1 as described above (Figure 5A; Adamczak et al., 2010; 
Nasrallah et al., 2014a; Shim et al., 2018). This was observed for medetomidine and ketamine-xylazine anesthesia. The additional patterns observed in contralateral thalamic relay nuclei and S2 at higher field strengths emphasize the somatosensory nature of the response along the spinothalamic tract (Jung et al., 2019). Weak responses in ipsilateral S1 have also been reported for some animals, likely due to inter-hemispheric projections (Schroeter et al., 2017; Shim et al., 2018; Jung et al., 2019), and no cardiovascular changes were observed during the stimulus periods in these studies (Figure 5B).

In contrast, the strong, transient cardiovascular surges in ventilated animals clearly indicate sympathetic activity, which is governed by medullary control areas and triggered by an autonomic stress response (Pfaff, 2005; Samuels and Szabadi, 2008b; Ulrich-Lai and Herman, 2009). Such a response involves activity of the locus coeruleus and other key nuclei of the brainstem and the forebrain arousal system, which innervate multiple cortical and subcortical areas (Figure 1A; Toussay et al., 2013; Lecrux and Hamel, 2016; Lecrux et al., 2019).

The specific reason for this strong sympathetic response in ventilated mice is not fully understood. It is known that endotracheal intubation and forced ventilation can induce excessive stress in humans and animals when the anesthetic depth is too low. We hypothesize that long stimulus trains in ventilated mice accumulate to engage the activity of subcortical arousal nuclei that drive the brain state gradually toward desynchronization (see "Metastability of Brain States, Hysteresis and Behavioral Monitoring" section; Hudetz et al., 2003; Solt et al., 2014; Vazey and Aston-Jones, 2014; Pal et al., 2018; Hayat et al., 2019). Elevated sympathetic activity causes a tightly coupled increase in cardiovascular and respiratory output (Pfaff, 2005; Ulrich-Lai and Herman, 2009). Yet, in ventilated mice, the respiration rate is fixed (to $80-90 \mathrm{bpm}$ ), which may lead to an allosteric mismatch ("prediction error") between autonomic command and sensory feedback (Alheid and McCrimmon, 2008; Kleckner et al., 2017). It is unclear whether this drives the animal's stress response. However, it appears that ongoing electrostimulation triggers an excitatory feed-forward loop that forces increased activity in reticulo-thalamo-cortical circuits, and causes bilateral patterns in the S1 barrel fields, and strong sympathetic outflow (Toussay et al., 2013; Lecrux and Hamel, 2016; Lecrux et al., 2019).

With isoflurane, this is a transient process, and mice usually fall back into stable sedation after a stimulation period, which is evident from HR and MABP traces (Schroeter et al., 2014; Reimann et al., 2018) as well as from behavioral observations in unparalyzed mice. Mice administered medetomidine while under ventilation have been shown to exhibit increases in their HRs progressively over the length of the fMRI scan following the first stimulation period (Schroeter et al., 2014). Medetomidine exerts its effects predominantly by inhibiting the locus coeruleus (Akeju and Brown, 2017). Once a competing mechanism engages this key arousal nucleus, the sedation is temporarily suppressed (Hayat et al., 2019), and the stimuli might be processed in the awakening, largely unaffected cortex (see " $\alpha 2$ AR Agonists Induce a Sleep-Like State by Suppressing General Arousal” section). Again, medetomidine produces a state from which arousal is more likely to occur than through the use of GABAergic drugs, which suppress the activity of excitatory neurons across the entire brain (Figures 1B,C).

Mechanically ventilated mice anesthetized with medetomidine exhibited bilateral BOLD responses even to single electrical pulses applied to the paw (1 mA, $0.5 \mathrm{~ms}$; Schlegel et al., 2015). The same single electrical pulses applied to ventilated mice under isoflurane, propofol, or urethane evoked BOLD responses contralaterally along the spinothalamic tract in S1, S2, and first-order TC nuclei at $9.4 \mathrm{~T}$-very similar to the patterns observed for stimulus trains in spontaneously breathing mice under ketamine-xylazine anesthesia at 15.2 T (Figure 5; Jung et al., 2019), with the latter being more spatially defined. The greatest similarities have been reported for patterns elicited under propofol along with profound hemodynamic responses to electrostimulation. However, the sedation under propofol was found to be unstable for the adjusted dosage (Schroeter et al., 2014; Schlegel et al., 2015). In summary, for the tested regimens, single pulses of electrical stimulation in mechanically ventilated mice elicit patterns predominantly along the somatosensory axis, whereas long pulse trains evoke bilateral patterns that likely reflect a transient recruitment of arousal structures, which can drive the brain state towards consciousness.

Multiple publications have reported on subcutaneous electrostimulation of the paw in mechanically ventilated mice under isoflurane. Whether this protocol allows for the detection of non-salient, low-intensity sensory stimuli has not yet been investigated. There is evidence that stimulus trains in the somatosensory range $(0.5 \mathrm{~mA})$ lead either to bilateral patterns or to no response at all (Shim et al., 2018). For a free-breathing sedation adjusted at $0.5-1 \%$ isoflurane, significant BOLD responses to whisker deflection have been reported in a murine fMRI study (Kahn et al., 2011). In such lightly sedated mice, tracheal intubation and forced ventilation would act as heavy continuous stressors during an fMRI experiment. Accordingly, mechanical ventilation does not permit further decreases in isoflurane concentrations, and approximately $1-1.3 \%$ are advised to prevent panic and withdrawal from the endotracheal tube. Concentrations of $0.75-1.1 \%$ isoflurane were found necessary to avoid burst suppression and permit stable sedation in spontaneous breathing mice (Kozai et al., 2015; Michelson and Kozai, 2018). To create conditions as close as possible to those experienced by a calm human volunteer, alternative strategies to ventilation should be considered for sensory tasks.

\section{Balancing Anesthesia in Spontaneously Breathing Mice}

Increasing oxygen concentration in the carrier gas is a popular strategy to prevent hypocapnia in spontaneously breathing mice, although caution is advised: no differences in respiratory rates, $\mathrm{pH}$, blood $\mathrm{CO}_{2}$ levels, or blood pressure have been found for mice breathing isoflurane in pure oxygen (100\%) compared to medical air (21\%). This has been explained by a decrease in lung volume for pure oxygen based on alveolar collapse, known as absorption atelectasis (Wilding et al., 2017). Hemodynamic responses to whisker stimulation were significantly reduced for mice breathing pure oxygen compared to medical air (Sharp 
et al., 2015). The exact concentration of oxygen in the breathing gas may be adjusted based on a bell-shaped stimulus-response curve with respect to the anesthetic protocol that is applied (Blazquez Freches et al., 2018).

A good strategy is to apply anesthesia that causes neither respiratory depression nor vasodilation. This makes $\alpha 2 \mathrm{AR}$ agonists attractive to assess sensory processing in murine fMRI, preserving physiological breathing rates at about 120-190 bpm (Adamczak et al., 2010; Nasrallah et al., 2014a). However, $\alpha 2 \mathrm{AR}$ agonists induce a sleep-like state and impair BOLD responses due to vasoconstriction (see above; Fukuda et al., 2013; Nasrallah et al., 2014a), which further initiates a transient increase in MABP followed by a reduction based on suppression of noradrenergic sympathetic ganglia (McCallum et al., 1998; Samuels and Szabadi, 2008a). A better choice might be the combination of two or more complementary anesthetics, to balance their respective actions and to tailor the desired effect (Fukuda et al., 2013). Combining the $\alpha 2 \mathrm{AR}$ agonist xylazine together with ketamine preserves physiological breathing rates at about $180 \mathrm{bpm}$ and leads to pronounced BOLD responses, at least in the contralateral S1 (Shim et al., 2018). Another promising alternative was introduced by balancing isoflurane anesthesia with fentanylfluanisone and midazolam, which allows the isoflurane level to be reduced to $0.5-0.8 \%$ (Sharp et al., 2015). This was reported to induce stable sedation and permit the detection of hemodynamic effects for whisker stimulation, with magnitudes and transition times similar to those observed in awake mice.

Balanced multimodal anesthesia holds great promise to circumvent the multiple challenges in murine fMRI, with the aim of preserving physiological conditions while sufficiently sedating the animal, and still ensuring a certain level of "connectedness" to sensory perception and interoception (see "Anesthetic Depth and Consciousness-the Virtue of Translational Neuroimaging" section; Sanders et al., 2012). Protocols for balanced multimodal anesthesia will be further discussed in "Multimodal Anesthesia in Translational fMRI" section.

\section{Nociception, Pain, and Physiological Confounds}

Nociception is "the neural process of encoding noxious stimuli." Pain is defined as an unpleasant experience that may or may not arise from nociception (Davis et al., 2017). In contrast to fMRI tasks that probe the central processing of applied stimuli, fMRI of pain explicitly aims to identify neural correlates of a phenomenal quality (Mouraux and Iannetti, 2018). Its assessment is therefore hampered in the (sedated) rodent, which cannot report on the experience or level of pain during an fMRI task (Seth et al., 2005). This is an important domain for translational fMRI: in principle, it should be possible to first correlate nociceptive-evoked BOLD patterns with subjective ratings of pain to identify pain-specific functional neurosignatures in the human brain (Wager et al., 2013; Woo et al., 2017). In a second step, a corresponding signature could be sought in the sedated animal model (Tracey, 2017). This leads to an even more fundamental question in terms of anesthesia: to what extent is consciousness required to encode a pain-specific neurosignature?
Currently, we cannot answer this question, since a pain-specific neurosignature has not yet been found (Mouraux and Iannetti, 2018). Painful stimuli are processed within a widely distributed network that is often referred to as the "pain matrix" (Legrain et al., 2011) - a term derived from Melzack's original neuromatrix theory of pain (see below; Melzack, 1989, 2005). It has been shown that salient and painful stimuli are processed by largely overlapping cortical areas in humans (Mouraux et al., 2011), and subcortical and arousal structures that are involved in nociception are also engaged by acute responses to stress (Reimann et al., 2016; Martins and Tavares, 2017). In other words, the neuromatrix is not pain-specific. This is a major obstacle for identifying pain-specific brain signatures, because painful stimuli are: (1) intrinsically salient (Legrain et al., 2011; Mouraux et al., 2011); and (2) often provoke autonomic stress responses (Reimann et al., 2016, 2018).

Such stress responses introduce severe complications in murine fMRI. They involve the activity of the

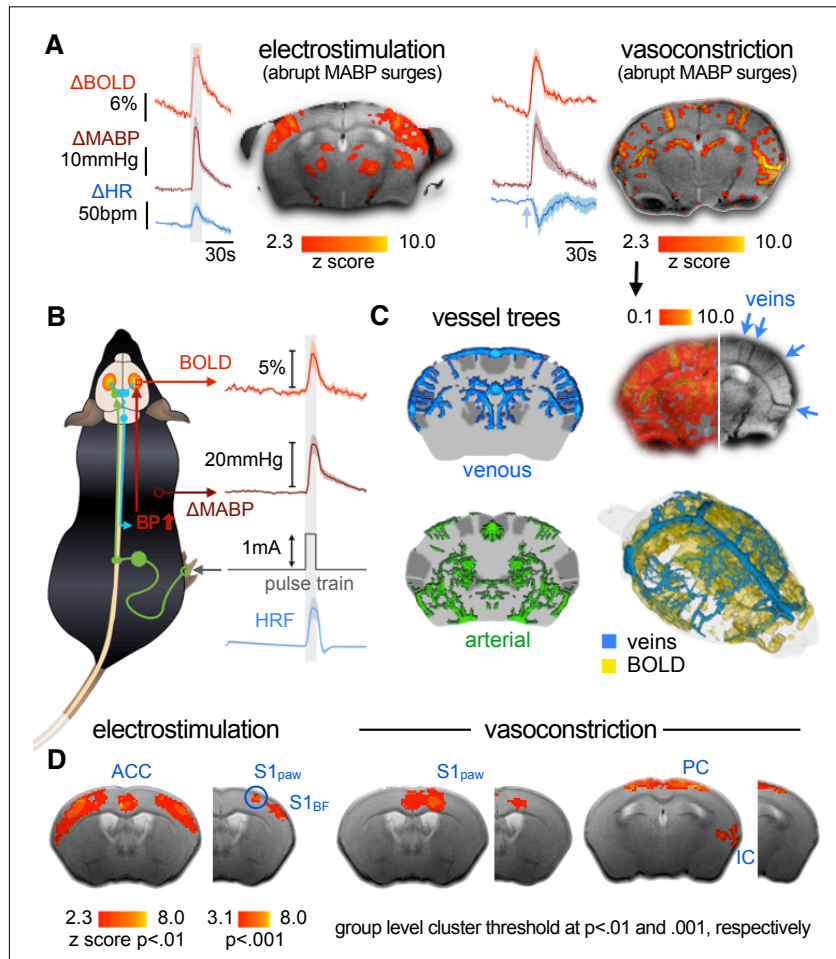

FIGURE 6 | BOLD patterns evoked by abrupt surges in MABP. (A) Electro-stimulation evokes abrupt surges in MABP and HR. Mimicking these surges by intravenous injection of vasoconstricting drugs leads to BOLD patterns co-locating with large veins. Injection time point is indicated by dashed line. (B) Nociceptive stimuli or stress can elicit abrupt surges in MABP that increase the influx of oxygenated blood into the brain vasculature. MABP and BOLD patterns are highly correlated with each other and with the applied stimulus. This causes the hemodynamic response function (HRF) - that is modeled based on the stimulus paradigm - to reveal MABP-induced effects as significant patterns. (C) Significant patterns co-localize with large veins and can therefore be identified and corrected for, whereas widespread patterns remain below the statistical threshold. (D) At the group level, significant effects persist at more liberal thresholds, and few minor clusters even at more conservative standards. Adapted with permission from Reimann et al. (2018). 
nociceptive-medullary-autonomic circuit-a fundamental component of the "flight or fight" response, which triggers abrupt surges in HR and MABP (Figure 6A; Price, 2000; Pfaff, 2005; Brown et al., 2010). Since cerebral autoregulation has high-pass filter characteristics, slow changes in MABP are buffered to a certain extent, whereas more pronounced and rapid changes are reflected in the CBF (Hamner et al., 2019). The efficiency of dynamic autoregulation might be further impaired by vasomodulatory effects of anesthesia (Lee et al., 1994; Dagal and Lam, 2009; Sanders et al., 2011). Abrupt surges in MABP, well within the physiological range ( $80-120 \mathrm{mmHg}$ in mice), can lead to stimulus-correlated increases in CBF causing widespread BOLD patterns across the brain (Figures 6B,C; Kalisch et al., 2001; Wang et al., 2006; Gozzi et al., 2007; Reimann et al., 2018). Veins are particularly prone to translate changes in $\mathrm{CBF}$, into pronounced BOLD effects due to their low oxygen concentration (Reimann et al., 2018). In fact, significant clusters were observed along large draining veins (cluster threshold $z=3.1$ ) that were highly correlated with transient MABP surges induced by methods of controlled pharmacological vasoconstriction (Figures 6A,D). Since large veins co-localize with key cortical regions of the neuromatrix (S1, S2, insula, and parietal cortex), this is a confounding factor in current murine fMRI studies of nociception. Aside from strategies to identify and correct the affected regions, fMRI protocols that track changes in $\mathrm{CBV}$, such as vascular space occupancy (VASO) or iron-oxide-based CBV-fMRI, are less prone to such large-vein effects (for a review, see Huber et al., 2017) and can be used either as alternative techniques, or to normalize and correct BOLD data.

Further complexity is introduced by outreaching vasodilatory projections of specific arousal nuclei such as the locus coeruleus, or the nucleus basalis of Meynert in the basal forebrain that are thought to have a substantial impact on the cortical hemodynamic responses to noxious stimuli (Lecrux and Hamel, 2016; Uchida and Kagitani, 2018; Paquette et al., 2019). The extent to which these BOLD responses actually reflect neural activity remains unclear (Lecrux et al., 2019). Both abrupt elevations in MABP and remote vasodilatory projections of arousal nuclei can produce hemodynamic readouts that are indistinguishable from neurovascular coupling. This problem has hardly been explored and is currently considered a major hurdle for interpreting experimental data related to nociceptive fMRI in (ventilated) rodents (Paquette et al., 2018).

Despite these considerations, nociceptive pathways are largely preserved in murine BOLD fMRI using low-dose mono-anesthetic isoflurane anesthesia in mechanically ventilated and paralyzed mice (Reimann et al., 2016). Brief heat stimuli just above the $\mathrm{A} \delta$ nociceptor threshold $\left(46^{\circ} \mathrm{C}\right)$ applied to the murine paw evoked BOLD effects in brain areas including the spinothalamic and spinoreticular tract, which have been well documented as having a functional involvement in murine and human nociception (Figure 7A). Employing a cryogenic transmit-receive unit (Niendorf et al., 2015) at 9.4 $\mathrm{T}$ has permitted resolving even the small nuclei of the habenular nociceptive pathway (Figure 7B, Reimann et al., 2016) - a pain modulating circuit known from human fMRI

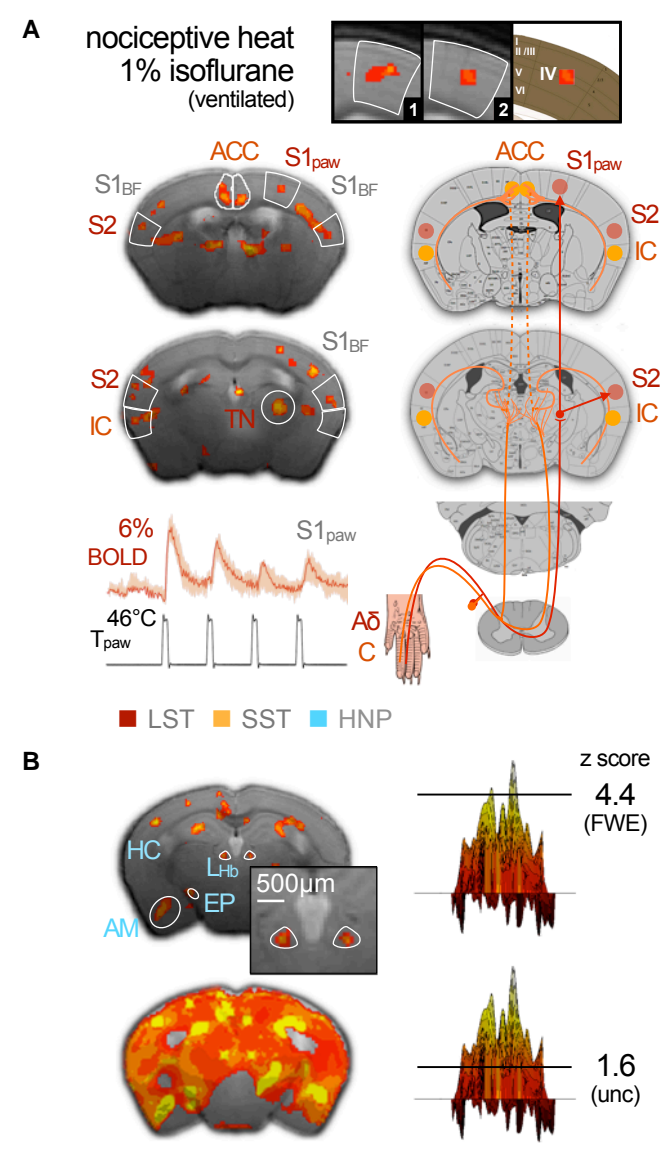

FIGURE 7 | Murine nociception induced by heat stimuli applied to the paw. (A) BOLD patterns along the spinothalamic (LST) and spinoreticular tract (SST) depict well-known nociceptive routes in the mouse with high spatial accuracy, including thalamic nuclei (TN), primary (S1) and secondary (S2) somatosensory cortex, the barrel field $\left(\mathrm{S}_{\mathrm{BF}}\right)$, and insular $(\mathrm{IC})$ and $\mathrm{ACC}$. (B) Involvement of the habenula nociceptive pathway (HNP) has also been observed, including amygdala (AM), hippocampal areas (HC),

entopenduncular nucleus (EP), and the small nuclei of the lateral habenula $\left(L_{\text {Hb }}\right)$. These highly precise patterns represented the tip of the iceberg of underlying BOLD effects, likely induced by MABP surges and vasodilative projections from subcortical arousal nuclei. Adapted from Reimann et al. (2016), http://creativecommons.org/licenses/by/4.0.

and from histological c-fos immunostaining in the mouse (Shelton et al., 2012a,b).

The detail of the BOLD patterns observed in this study was achieved by highly conservative thresholding, and has exposed peak values of extensive patterns spreading across large parts of the brain (Figure 7B). More liberal thresholding of heat-evoked BOLD effects in mice under comparable conditions revealed pattern distributions very similar to those observed for electrostimulation (Bosshard et al., 2015). In fact, nociceptive and arousal pathways are tightly interconnected (Craig, 2013). It is thus difficult to disentangle or exclude a potential contribution of ventilation-driven stress responses from those involved in nociception (as discussed in "Sensory Processing and the Key Challenges in Murine fMRI" section). Nociception drives arousal pathway activity, and hence cortical desynchronization, toward a 
transient emergence from anesthesia (Figure 2E; Hudetz et al., 2003; Solt et al., 2014; Vazey and Aston-Jones, 2014; Akeju and Brown, 2017; Sanders, 2017; Hayat et al., 2019). Therefore, it is important to develop protocols that permit stable sedation in receptive, spontaneously breathing animals. The continuous monitoring of MABP permits the tracking of this important confounder, but it is highly invasive and introduces postoperative nociception. Sympathetic activity is similarly reflected in HR and respiration. Both may rise with the stimulus, but should subsequently return to the baseline (Reimann et al., 2016, 2018). A progressively rising $\mathrm{HR}$ requires adjustment of the anesthetic dosage to avoid the animal waking (Nasrallah et al., 2014b; Schroeter et al., 2014; Shim et al., 2018).

The interconnection of nociceptive and arousal routes causes a transient lightening of anesthesia in response to nociceptive stimuli. This could explain why BOLD responses to noxious electrostimulation are partially preserved in humans even after propofol-induced LOC, whereas sensory evoked patterns vanish (see "Anesthetic Depth and Consciousness-the Virtue of Translational Neuroimaging” section; Lichtner et al., 2018). In relation to our initial question, of whether consciousness is required to evoke a pain-specific neurosignature, it could be said that nociception itself drives the brain state toward consciousness. The proposed architecture of the body-self neuromatrix is composed of sensory, affective, and cognitive subnetworks of brain areas. Their output patterns produce the multiple dimensions of the pain experience (sensorydiscriminative, cognitive-evaluative, motivational-affective), which coincide with homeostatic and behavioral responses (Melzack, 2005; Craig, 2013; Seth and Tsakiris, 2018). Dynamic changes in the FC of these respective networks, triggered either by external nociceptive stimuli or by internal processes in animal models of chronic pain, may offer a more concrete representation of the neuromatrix, as was recently addressed as the dynamic pain connectome (Kucyi and Davis, 2017).

\section{Functional Connectivity and Murine Resting-State fMRI}

FC between two or more brain areas is defined by the correlation coefficient of their signal intensity courses over time: the higher the correlation, the higher the FC (Figure 8A; Petersen and Sporns, 2015). This simple relation is the key principle in defining functional networks across the entire brain-an opportunity that is unique to fMRI. FC is considered to be in large part the hemodynamic translation of simultaneous activity of neural populations, which is inherently limited by the physical connections between neurons (Mateo et al., 2017). This structural scaffold permits a repertoire of possible functional configurations that changes dynamically on a time scale depending on the level of organization: on the order of $1 \mathrm{~ms}$ for neurons, $10 \mathrm{~ms}$ for local circuits, $100 \mathrm{~ms}$ for EEG, and $1 \mathrm{~s}$ for fMRI (Mashour and Hudetz, 2018). This has two important implications: (1) the temporal changes in FC hold important information that is simply discarded when large fMRI time series are averaged assuming steady-state conditions (Preti et al., 2017); and (2) brain states can, in principle, be translated into FC and assessed via fMRI (Van de Ville et al., 2010).

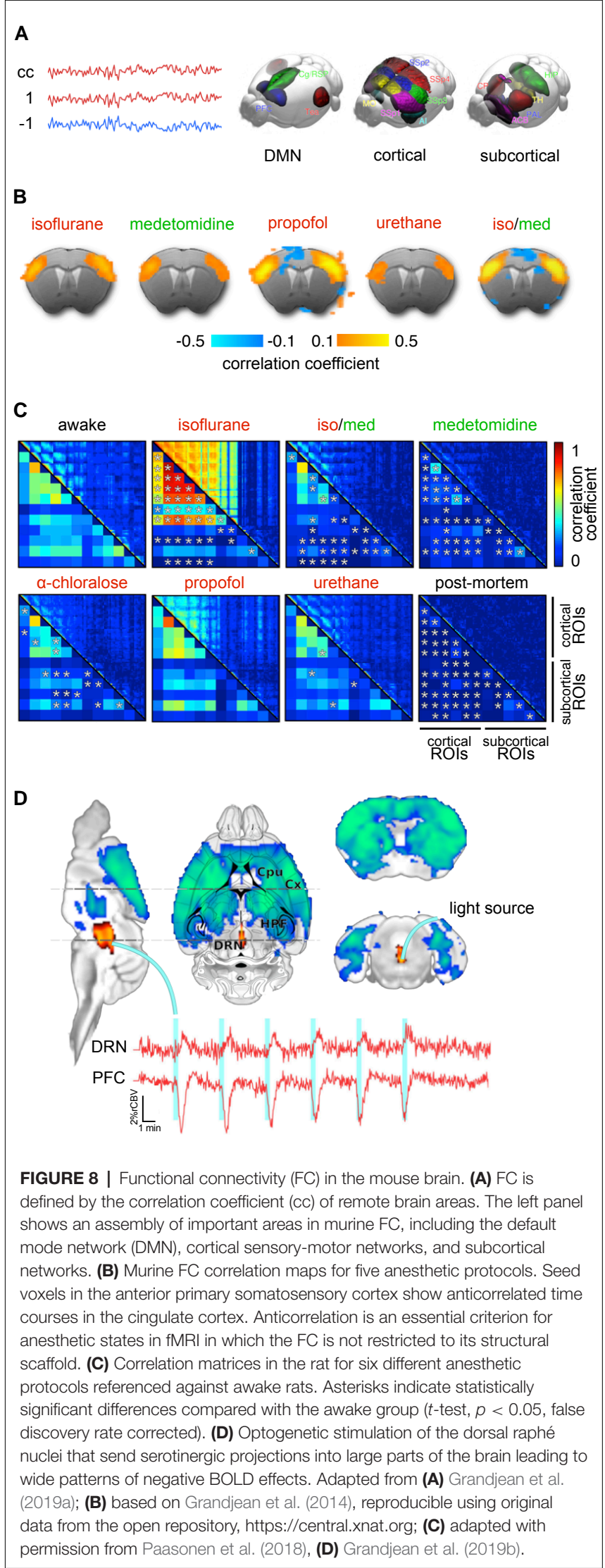


The integrity or disruption of FC is commonly used as an indicator of various types and stages of neurodegenerative diseases and neurophysiological malfunctions, or to study the effects of pharmacological interventions, genetically modified proteins or neural populations under optogenetic control (see "Deep Brain Stimulation, Opto- and Chemogenetics" section). Notably, the majority of murine resting-state studies have been conducted under anesthesia (Jonckers et al., 2011, 2014, 2015; Guilfoyle et al., 2013; Grandjean et al., 2014; Mechling et al., 2014; Nasrallah et al., 2014a; Sforazzini et al., 2014; Liska et al., 2015; Bukhari et al., 2017; Wu et al., 2017; Pan et al., 2018). The extent to which various anesthetic protocols intrinsically disrupt FC is therefore a critical issue that must be comprehensively addressed. A number of excellent reviews are already available on this issue for humans (Mashour and Hudetz, 2017, 2018) and mice (Hoyer et al., 2014; Jonckers et al., 2014, 2015; Pan et al., 2015; Gozzi and Schwarz, 2016; Chuang and Nasrallah, 2017; Sumiyoshi et al., 2019; Grandjean et al., 2020; Mandino et al., 2020). Here, we add to the existing literature by focusing on basic concepts of anesthesia-specific changes in FC with respect to the underlying mechanisms.

FC is remarkably similar across mammalian species. In 2014, 3 years after the first study on murine FC (Jonckers et al., 2011), it was shown that mice exhibit a medial fronto-parietal or "default mode" network (DMN) during the resting state (Stafford et al., 2014) similar to that of rats (Lu et al., 2012), monkeys (Vincent et al., 2007), and humans (Raichle et al., 2001). The DMN plays a fundamental role in baseline functions of the mammalian brain (Raichle, 2015). In humans, it is associated with mind-wandering and rumination. Whether the DMN entails comparable phenomenal qualities in mice remains speculative and may be interesting from an evolutionary or philosophical point of view (Seth et al., 2005; Havlik, 2017). In the presence of a salient stimulus, DMN connectivity becomes disrupted and shifts towards connectivity in the dorsal attention network (Raichle, 2015), in the same way that cortical states change with attention toward a stimulus (see "Cortical States of Wakefulness" and "Brain States Under Anesthesia" sections).

The DMN involves similar higher-order cortical regions along the medial fronto-parietal axis across species (Figure 8A; Raichle, 2015; Grandjean et al., 2020), although prefrontal and cingulate regions appear to perform the tasks of the precuneus, which is absent in rodents (as reviewed in Gozzi and Schwarz, 2016). Further functional networks have been described in the mouse, including a salience network and latero-cortical network, as well as various sensory, sensorimotor, cerebellar, limbic system, and basal ganglia networks (see Figure $\mathbf{8 B}$ for murine $\mathrm{FC}$ maps based on a seed voxel in S1 for different anesthetics; Jonckers et al., 2011, 2014; Grandjean et al., 2014, 2017b, 2020; Nasrallah et al., 2014b; Sforazzini et al., 2014; Zerbi et al., 2015; Shim et al., 2018; Gutierrez-Barragan et al., 2018).

\section{Anesthesia-Induced Disruptions in FC}

The network architecture is very similar between humans and mice (Bullmore and Sporns, 2012; Gămănuț et al., 2018; Balsters et al., 2019; Fulcher et al., 2019), which has been utilized to pursue questions about human network configurations in the mouse (Fulcher and Fornito, 2016). Brain networks are organized around highly connected hubs. Substantial disruption of FC induced by anesthesia at these central nodes can cause widespread communication failure associated with LOC (Schröter et al., 2012; Lee H. et al., 2013; Moon et al., 2015; Bonhomme et al., 2016; Tononi et al., 2016; Mashour and Hudetz, 2018). Abnormal reorganization of hubs during recovery from anesthesia likely explains differences in FC strength and configuration observed prior to and following LOC (Långsjö et al., 2012; Liu et al., 2013b; Monti et al., 2013). This sheds light on the anesthetic hysteresis effect: namely, that different anesthetic dosages are required to induce a similar depth of anesthesia during the induction and recovery phases (see "Brain States Under Anesthesia" section). The translational utility of murine FC studies may depend on the capacity of the anesthetic protocol to preserve or restore the integrity of specific functional networks across the brain.

Of course, the technical equipment, animal preparation protocols, arousal states due to stress, murine susceptibility to vascular effects of anesthesia, and the applied statistical methods all have a crucial impact on the reliability of the data (Grandjean et al., 2020). It is therefore helpful to reference murine FC data against: (1) FC data derived from more stable systems like humans, monkeys, or rats under the same anesthesia; and (2) the molecular pathways that are known to be targeted by specific anesthetic protocols. Disruption of neural communication across brain areas occurs according to the principal target routes of an anesthetic regimen (see "Brain States Under Anesthesia" section) and may be translated into a discrete FC signature (Table 3). This relation makes the assessment of FC a highly relevant point of convergence for detailing and understanding brain states induced by anesthesia.

\section{Anticorrelation and Rich Dynamic Repertoires-Criteria for Appropriate FC}

Discrete signatures of GABAergic drugs and volatile ethers, which suppress brain-wide neural activity include dose-dependent disruption of FC across the entire brain (for details, see Table 3; Boveroux et al., 2010; Schröter et al., 2012; Jordan et al., 2013; Ní Mhuircheartaigh et al., 2013; Palanca et al., 2015; Bonhomme et al., 2016; Ranft et al., 2016). This is reflected in a reduction in global connectedness, with stronger effects on higher-order cortical and thalamic FC compared to sensory areas, and fewer transitions between functional networks (Uhrig et al., 2018; Golkowski et al., 2019). This rigidity in the dynamics between networks correlates with a reduced ability to interact with external stimuli (see "GABAA Agonists Suppress Neural Activity Across the Central Nervous System" section). LOC has consistently been found to be correlated with a breakdown of fronto-parietal FC in humans and non-human primates induced by GABAergic drugs (Purdon et al., 2009; Boveroux et al., 2010; Jordan et al., 2013; Monti et al., 2013; Palanca et al., 2015; Ranft et al., 2016; Mashour and Hudetz, 2017; Uhrig et al., 2018), likely due to impaired frontal-to-parietal cortical information transfer ( $\mathrm{Ku}$ et al., 2011; Jordan et al., 2013; Ranft et al., 2016). Although this relationship is also 
The table summarizes key effects on FC for the three major anesthetic classes: disruption of whole brain FC by GABA $A_{A}$ receptor agonists (GABA $A^{+}$, red), bottom-up disruption by a2 adrenoreceptor agonists ( $\alpha 2_{R}+$, green), and disruption of cortical FC by NMDA receptor antagonists (NMDA $A_{R}-$, blue). Loss of consciousness (LOC) is most likely mediated by suppression of frontal-to-parietal information transfer in the cortex and can be achieved by all three anesthetic classes either directly via disruption of cortico-cortical FC or indirectly via bottom-up pathways. Principal mechanisms of anesthesia are described in "Brain States Under Anesthesia" section and their reflection in FC patterns is discussed in "Functional Connectivity and Murine Resting-State fMRl" section. References are shortened to first author (and year) to save space and allow for comprehensive referencing.

thought to exist in mice, further studies are required to assess the modes of murine fronto-parietal FC and their significance for wakefulness (Imas et al., 2005b; Mashour and Alkire, 2013; Sforazzini et al., 2014; Pal et al., 2018; Shofty et al., 2019; Grandjean et al., 2020).

On the other hand, burst suppression and slow waves that propagate unabated across the cortical surface (see "Crossing the Borders: GABAergic Slow Waves and Burst Suppression" section) promote FC that can span extensive cortical networks in humans and rats, occasionally reaching into the basal ganglia and thalamic structures (Liu et al., 2011, 2013b,c; Golkowski et al., 2017; Schwalm et al., 2017; Aedo-Jury et al., 2019). Isoflurane concentrations as low as $1.3 \%$ in ventilated rats were found sufficient to induce highly synchronized large-scale FC patterns in fronto-cortical and striatal regions, and to suppress most thalamo-cortical and intra-subcortical connections (Figure 8C; Paasonen et al., 2018).

Loss of information transfer and integration is further reflected in a reduced number of possible FC configurations
(Hutchison et al., 2014; Barttfeld et al., 2015; Hudetz and Mashour, 2016; Ma et al., 2017; Uhrig et al., 2018). The repertoire of FC configurations can be evaluated via a sliding-window approach. In this approach, a window length of about $1 \mathrm{~min}$ is defined and shifted along the entire length of the resting-state time series, with a step size equal to the fMRI temporal resolution (typically 1-2 s). Each time-shifted segment then undergoes a correlation analysis, which gives access to dynamic aspects of FC, which have been reported to be well conserved across rats, monkeys, and humans (Majeed et al., 2011). Although blurred in temporal resolution, this technique permits the assessment of dynamic whole-brain signatures based on FC. Combining $\mathrm{Ca}^{2+}$ imaging or electrophysiology with fMRI (Thompson et al., 2013; Keilholz, 2014; Schmid et al., 2016; Schwalm et al., 2017; Lurie et al., 2018; Schlegel et al., 2018; Matsui et al., 2018; Aedo-Jury et al., 2019; van Alst et al., 2019), these signatures can be linked to the cortical states, and hence be employed to determine the nature and level of sedation (see "Brain States Under Anesthesia" and "Limitations in Maintaining Stable Brain States" sections). 
The dynamical elaboration of a rich, flexible repertoire in the awake brain becomes increasingly constrained with increasing anesthetic dosage (Barttfeld et al., 2015; Uhrig et al., 2018). This is accompanied by a lack of anticorrelated areas-a further symptom of inflexible FC and confined information traffic pointing to LOC. A lack of anticorrelated areas was reported in mice ventilated at $1 \%$ isoflurane (Figure 8B; Grandjean et al., 2014). At 1.3\% isoflurane broad cortical FC has been observed along with synchronized cortical slow wave activity in the $\mathrm{Ca}^{2+}$ signal assessed in the murine S1 (Schlegel et al., 2018). Further reducing the isoflurane concentration below $1-1.3 \%$ is not appropriate if the mice are ventilated and paralyzed (see "Sensory Processing and the Key Challenges in Murine fMRI" section). As head movements, including rhythmic, respirationinduced motion, account for a large part of the noise in FC (Kalthoff et al., 2011; Pais-Roldán et al., 2018), a trade-off has to be made between data quality and anesthetic depth. In ventilated rats, a reduction of isoflurane from $1.5 \%$ to $1 \%$ was sufficient to preserve distinct cortical networks and anticorrelated FC at a largely desynchronized frequency band in the EEG (Liu et al., 2013c). Due to the narrow dose range between stable sedation and burst suppression, careful adjustment of isoflurane concentrations is required to avoid unphysiological FC.

Compared to isoflurane and other volatile ethers, halothane exhibits a remarkably wide dose range between stable sedation and burst suppression (Murrell et al., 2008; Brown et al., 2018; McIlhone et al., 2018). A rich repertoire of dynamic FC patterns was observed for halothane-anesthetized mice, based on a varied set of recurring configurations of FC, with abundant anticorrelated areas (Sforazzini et al., 2014; Gutierrez-Barragan et al., 2018). At low dosages, other GABAergic drugs have been shown to preserve FC quite well with respect to the above criteria. The use of low-dose propofol and urethane was reported to achieve the best resemblance to FC in awake rats out of six different anesthetic regimens tested (Figure 8C; Paasonen et al., 2018). A similar observation was reported for mice, with propofol preserving spatially defined symmetrical cortico-cortical FC with anticorrelated cingulate areas (Figure 8B; Grandjean et al., 2014). Nevertheless, unstable sedation has been reported for this protocol in mice, which could be due to ventilation conditions, considering the general arousability of mice relative to rats. Comparing the capacity of anesthetic protocols to approximate the FC of the awake state in rats, propofol and urethane showed the best performance, followed by $\alpha$-chloralose, while a protocol combining isoflurane and medetomidine (see below) was ranked 4th (Figure 8C).

\section{“One-Dimensional” Disruption of FC}

Medetomidine and other $\alpha 2 \mathrm{AR}$ agonists, which suppress subcortical arousal nuclei (see "Brain States Under Anesthesia" section) disrupt FC in a bottom-up manner (Table 3). Disinhibition of sleep-promoting cells in the hypothalamus disrupts subcortico-subcortical and hence thalamo-cortical FC. This causes a discrete FC signature that reflects the functional disconnection of the cortex from subcortical areas, as anticipated on the basis of the cerveau isolé ("Brain States Under Anesthesia" section; Bremer, 1935; Guldenmund et al., 2017; Lewis and Akeju, 2017; Akeju and Brown, 2017). With increasing dosages, local cortical activity gets coupled with slower rhythms of subcortical afferents from the suppressed arousal nuclei, building uncoupled islands across the cortex (Nir et al., 2011; Lewis et al., 2012). This is reflected in disrupted interhemispheric cortico-cortical FC, which has been observed with increasing dosages of medetomidine in rats (Nasrallah et al., 2014a) and mice (Nasrallah et al., 2014b). The mechanism of bottom-upinduced LOC likely involves a breakdown of FC between the locus ceruleus, the thalamus, and the posterior or anterior cingulate cortex, which may eventually affect fronto-parietal FC (Långsjö et al., 2012; Fernandez et al., 2017). Medetomidine was found to preserve moderate intercortical connectivity in ventilated rats, although this was significantly reduced compared to that of awake rats, whereas thalamo-cortical activity was almost absent (Figure 8C; Pawela et al., 2008; Zhao et al., 2008; Paasonen et al., 2018).

Ketamine, on the other hand, which acts primarily in a top-down manner in the cortex, mediates LOC by direct suppression of fronto-parietal and further cortico-cortical FC (Table 3; Voss et al., 2012; Lee U. et al., 2013; Blain-Moraes et al., 2014; Muthukumaraswamy et al., 2015; Bonhomme et al., 2016; Vlisides et al., 2017; Mashour and Hudetz, 2018; Uhrig et al., 2018). In contrast to GABAergic and $\alpha 2 \mathrm{AR}$ agonists, ketamine has been reported to largely preserve subthalamic FC, which is consistent with its activating effects on the preoptic area and other wake-promoting nuclei (Alkire and Miller, 2005; Mashour, 2014). The top-down and bottom-up actions of ketamine and medetomidine may be thought of as "one-dimensional" (Mashour and Hudetz, 2017). The dual effect of GABAergic drugs makes them more suitable for stable sedation by suppressing both subthalamocortical and cortico-cortical FC (Boveroux et al., 2010). To achieve a similar anesthetic stability by not overly stressing one pathway, drugs targeting either of these "onedimensional" mechanisms are commonly combined (e.g., ketamine-xylazine or medetomidine-isoflurane) in order to target the complementary pathways.

\section{Multimodal Anesthesia in Murine Resting-State fMRI}

Combining isoflurane and medetomidine at levels roughly half their mono-anesthetic dosages (Lu et al., 2012; Paasonen et al., 2018; Sumiyoshi et al., 2019) has been shown to produce FC resembling that of awake rats substantially better than either mono-anesthetic protocol alone, yielding a correlation matrix that roughly corresponds to that of $\alpha$-chloralose (Figure 8C; Paasonen et al., 2018). This medetomidine-isoflurane protocol was adapted for murine resting-state fMRI, where it was also described as providing the "best of both worlds": reproducible, spatially defined networks, including symmetrical patterns of FC in bilateral sensory areas anticorrelated with cingulate regions, at a stable sedation in ventilated mice (Figure 8B; Grandjean et al., 2014).

Later studies revealed a high correspondence between FC and structural connectivity of cortico-cortical and cortico-striatal regions with the same medetomidine-isoflurane protocol in 
mice, whereas thalamo-frontal cortical FC was found suppressed (Grandjean et al., 2017b). Although a high correspondence between functional and structural connectivity has been associated with a constrained repertoire of FC configurations (Barttfeld et al., 2015; Uhrig et al., 2018), the sliding-window approach ultimately revealed rich patterns of dynamic FC under this protocol (Grandjean et al., 2017a). This incongruity could be due to methodological differences between the studies (e.g., the use of MR diffusion-based vs. neural tracerbased structural connectivity; Straathof et al., 2019). The exact relationship between the dynamics of FC and its correspondence to its scaffold as a function of anesthetic depth remains to be clarified across anesthetic protocols in mice.

Taken together, the preservation of a rich repertoire of FC configurations, along with spatially defined and anticorrelated FC in relevant networks, has made medetomidine-isoflurane the protocol of choice for studying murine FC. Low-dose halothane performs similarly well in mice, with the advantage of preserving thalamo-frontal cortical FC (Gutierrez-Barragan et al., 2018; Bertero et al., 2018; Mandino et al., 2020). Halothane is rarely used due to a risk of causing liver damage, although the risk is possibly no higher than that of other halogenated vapors (Mizobe, 2019).

A recent meta-analysis comparison across numerous labs, MR scanners, and animal preparation protocols (Grandjean et al., 2020) revealed greater specificity within elements of the DMN under medetomidine-isoflurane anesthesia in ventilated mice than in awake habituated mice, although the latter exhibited higher overall FC. This can be interpreted as an effect of environmental or internal distraction in awake mice, since DMN connectivity is known to be disrupted in the presence of salient stimuli (see above; Raichle, 2015). Contradictory findings have been reported for anesthetic effects on the DMN, although it appears that FC is principally supported at low anesthesia and disrupted with increasing anesthetic depth (Akeju et al., 2014a; Huang et al., 2014; Palanca et al., 2015; Ranft et al., 2016).

\section{Resting-State fMRI in the Awake Mouse}

Medetomidine-isoflurane and low-dose halothane are currently considered the most suitable anesthetic protocols available for the assessment of murine FC. Yet, carefully titrated multimodal protocols specifically designed to balance the complementary effects of their anesthetic compounds offer far more room for improvement (see "Multimodal Anesthesia in Translational fMRI" section). Comparing FC matrices for medetomidineisoflurane against propofol with reference to the "ground truth" of an awake habituated rat gives an immediate impression of what we are heading for (Figure $\mathbf{8 C}$ ). With respect to the nonvoluntary task conundrum, resting-state applications have the best chance to produce reliable data from awake habituated mice. Nevertheless, training awake mice to tolerate the noisy scanner environment is far more challenging than for awake rats (Jonckers et al., 2014, 2015; Low et al., 2016b; Dopfel and Zhang, 2018), although further strategies are constantly being developed to facilitate this procedure (Yoshida et al., 2016; Madularu et al., 2017; Han et al., 2019; Chen et al., 2020).
Since brain states during wakefulness alter with the level of arousal, such a "ground truth" is still prone to reflect stress and restraint rather than the resting state, which becomes critical when comparing results with human volunteers (Bergmann et al., 2016). It might be useful to administer minimal anesthetic dosages (e.g., of anxiolytics) in the actual experiment to relax mice, which are already habituated. This would create a state of drowsy wakefulness that approximates the "ground truth" against which novel anesthetic protocols could be referenced. Ultimately, multimodal sedation may be instrumental to lock the mouse into a defined spectrum of brain states to minimize variations due to stress, arousal, and distraction, and to prevent effects due to habituation and reward.

\section{Deep Brain Stimulation, Opto- and Chemogenetics}

Functional reorganization and network properties can be further evaluated by using techniques that modulate neural activity from within the brain. The most popular methods are deep brain stimulation, opto- and chemogenetics. These techniques allow one, for example, to evaluate the reorganization of FC in response to locally targeted perturbations. This can be used as a measure for functional complexity of information processing in the anesthetized brain (perturbational complexity index; Casali et al., 2013; Mashour and Hudetz, 2018). Ketamine, for instance, has been shown to preserve cortical functional complexity, which is decreased by propofol with increasing dosage (Sarasso et al., 2015). The signal propagation of locally induced neural activity also provides useful information on FC, which has been shown to be impaired during anesthesia, sleep, and pathological unconsciousness (Massimini et al., 2005; Ferrarelli et al., 2010; Casali et al., 2013). In humans, such investigations are often limited to studying cortical FC using non-invasive transcranial magnetic stimulation. In preclinical fMRI deep brain stimulation can be more easily utilized to induce neural activity in subcortical areas (McIntyre and Anderson, 2016), although the electrodes are prone to cause imaging artifacts, distortions, and local signal loss (Lehto et al., 2018).

Optogenetics employs optical fiber probes that do not directly interfere with functional MR image acquisition. Excitation or inhibition of genetically modified neurons governed by light control can be combined with fMRI to detail spatial and temporal effects on evoked neural activity (Desai et al., 2011; Lai et al., 2015) or FC in remote brain regions (Ryali et al., 2016; Chan et al., 2017). Chemogenetics is a powerful minimally invasive complement to optogenetics, in which neurons are controlled by artificially designed ligands that are injected into the bloodstream (Roth, 2016). Both are important techniques to further investigate the mechanisms of FC (as recently summarized in Mandino et al., 2020), including the impact of distinct transmitter systems in mice. Exclusively selecting, for example, serotonergic neurons of the raphé nuclei (Figure 8D; Grandjean et al., 2019b) or noradrenergic neurons of the locus coeruleus (Zerbi et al., 2019) based on specific gene expression patterns can be used to detail the underpinnings of brain states 
in anesthesia and the circuits that drive awakening (Carter et al., 2010; Taylor et al., 2016).

\section{TRANSLATIONAL fMRI ACROSS BRAIN STATES AND SPECIES}

The key challenges of preclinical fMRI with a view to translation are: (1) to induce and maintain brain states that correspond with those of calm and relaxed human volunteers; and (2) to assure hemodynamic integrity-in other words, to preserve neurovascular coupling and physiological baseline conditions. Up to this point, we have discussed the general principles of anesthesia and have highlighted the need to recognize and overcome the shortcomings of current anesthetic protocols. This will require strategies to shape, maintain, and unambiguously identify distinct brain states in fMRI that meet the above criteria, to conduct reliable, reproducible studies and translate findings from mice to humans.

\section{Multimodal Anesthesia in Translational fMRI}

Preclinical fMRI aims for an animal that is sufficiently sedated, yet with a fully functional brain. This challenge puts high demands on anesthetic protocols to induce and maintain an intermediate brain state that closely approximates an awake, calm, relaxed, and undistracted state during the fMRI task. Current standard practices involving simple mono-anesthesia are outdated. Balanced multimodal anesthesia, in which two or more drugs are combined to balance their complementary effects, has come into use in the clinic (Brown et al., 2018a) and should be further developed for preclinical studies (see "Sensory Processing and the Key Challenges in Murine fMRI" and "Functional Connectivity and Murine Resting-State fMRI" section; Grandjean et al., 2014; Sharp et al., 2015; Shim et al., 2018).

By reducing the dose of individual compounds, a multimodal protocol reduces excessive stress on a single pathway, taking advantage of complementary modes of action to shape and maintain desired brain states. In principle, respiratory depression or hemodynamic side effects from specific drugs can be attenuated by balancing them with the effects of others. At the same time, combining anesthetic compounds through multimodal anesthesia introduces a higher level of complexity and potential cross-effects that require caution. A rigorous characterization of anesthetic actions will be necessary to understand and adjust the effects of individual drugs in multimodal approaches. This will require that brain states, hemodynamics, and physiology be monitored, evaluated, and reported to the community.

\section{Defining Brain States in fMRI: Qualifiers and MIND Signature}

To properly identify and communicate an anesthetic state in translational fMRI, we first need to define a set of functional characteristics that can identify an induced brain state with high specificity. Their purpose is to evaluate the anesthetic maintenance of an intermediate brain state, and qualify its approximation to the awake state. We will refer to these functional characteristics as qualifiers.

Among these qualifiers, we choose one identifier that allows the unambiguous assessment of whether a desired intermediate brain state has been maintained during the full length of the fMRI scan, based on a reliable neurophysiological signature pattern. It should permit: (1) identifying a brain state and the integrity of its qualifiers; and (2) comparing this state across subjects, trials, scanners, labs, and species. We will refer to this identifier of a specific brain state as a maintained, intermediate neurophysiologically-determined (MIND) signature.

\section{Qualifiers}

A number of qualifiers have the potential to illuminate characteristics of brain states and the functional implications that accompany them. The set of such qualifiers is growing rapidly through new findings and the development of novel analytical tools (as summarized in Bonhomme et al., 2019). Here, for the sake of brevity, we list only those that we consider most relevant and sufficient for defining brain states for fMRI in anesthetized animals.

Under these conditions, the set of functional characteristics needed to identify an anesthetically induced brain state should include measures of maintenance, arousability, neuronal stimulus-response features, and their hemodynamic translation, as well as FC and its dynamic repertoire (Figure 9). These qualifiers cover a range of informative features to identify and evaluate the degree to which an induced brain state approximates the calm, awake state.

Cortical EEG signatures provide valuable information about cortical synchronization at particular frequency bands (including correlates of phase-amplitude coupling, which can influence cortical information transfer and sensory processing; see "Brain States Under Anesthesia" section; Chamadia et al., 2019), the stability of a maintained intermediate brain state during rest and its response to nociception and stress (see "Metastability of Brain States, Hysteresis, and Behavioral Monitoring" section). Due to its real-time accessibility and high temporal resolution, EEG can be used to assess anesthetic depth and rousability, particularly when aligned with behavioral observations (outside the scanner).

The dynamics of neural stimulus-responses can be directly assessed via EEG (whose spatial resolution is limited due to the smoothing properties of the scalp and skull) or invasive electrophysiological recordings of SEP and $\mathrm{Ca}^{2+}$ signals in primary, secondary sensory, and higher-order cortical areas. Multichannel recordings (Muller et al., 2018) and large-scale assessment techniques of neural activity, including high-density EEG (Massimini et al., 2005, 2007), $\mathrm{Ca}^{2+}$ - and voltage-sensitive dye imaging (McVea et al., 2016), can be used to assess the degree of complexity of signal processing and propagation across the cortex at different spatial and temporal scales (see also "Brain States Under Anesthesia" and "Deep Brain Stimulation, Optoand Chemogenetics" sections). This provides insights into the effects of anesthesia on thalamo-cortical and cortico-cortical sensory transmission, as well as cortical information transfer (see "Brain States Under Anesthesia" section). 


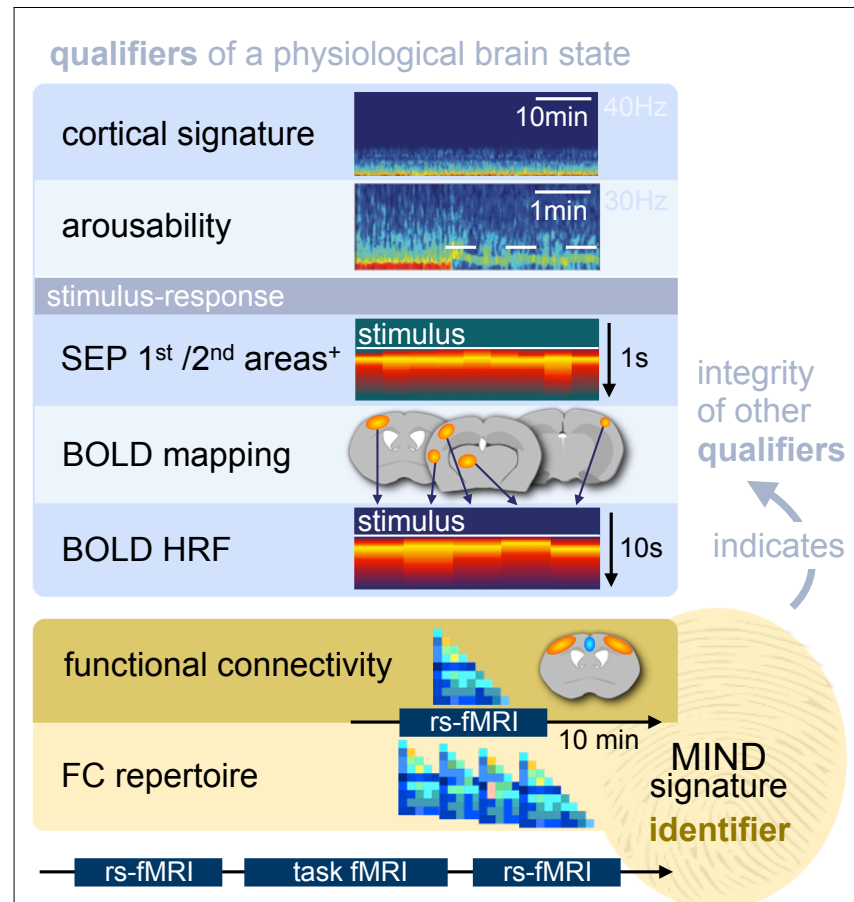

FIGURE 9 | The functional characteristics (qualifiers) and MIND signature (identifier) of a specific brain state induced by an anesthetic protocol. The aim of anesthesia in $\mathrm{fMRl}$ is to induce stable sedation, while maintaining a brain state that approximates an awake, calm state. The protocol may be either mono-anesthetic (drug a; dose $\mathrm{x}$ ) or multimodal (drug a, b, c; dose x, y, z). Qualifiers are defined as functional characteristics of an intermediate brain state that has been induced and maintained by the use of anesthesia: The cortical EEG signature permits to monitor anesthetic depth, to evaluate arousability by challenging the stability of a brain state via nociception or stress, and to probe stimulus-response features of sensory evoked potentials (SEP). Hemodynamic translation can be evaluated via fMRI, whereas shape and magnitude of the hemodynamic response function (HRF) provide information about anesthesia-induced vasomodulation. Anesthetic effects on functional connectivity (FC) across the brain can be assessed based on correlation matrices that depict interrelations of cortical and subcortical areas, including anticorrelations, and the dynamic repertoire of recurring sets of FC patterns over time. FC has great value as an identifier of a particular brain state and the integrity of its qualifiers. A fingerprint-like FC signature pattern can be used to unambiguously identify maintained intermediate brain states under anesthesia and communicate them across labs. This signature pattern is termed the maintained, intermediate neurophysiologically-determined (MIND) signature. Flanking tasks in fMRI with resting-state scans will permit a researcher to monitor the maintenance or variations in the brain state under anesthesia throughout the fMRI experiment.

Hemodynamic integrity can be evaluated via the translation of neural responses into BOLD effects. Comparing SEP or evoked $\mathrm{Ca}^{2+}$ signals with BOLD effects reveals whether neural responses are triggered but become lost in hemodynamic translation (Aksenov et al., 2015). Neurovascular coupling varies across cortical areas and has been found to be more reliable in sensory vs. higher-order cortices (Ojemann et al., 2002, 2013; Conner et al., 2011). Therefore, an overall impairment of neurovascular coupling by anesthesia might lead to a selective loss of BOLD effects. Further information about vasoconstrictive or vasodilatory drug effects can be derived from the shape of the hemodynamic response function (HRF; see "Sensory Processing and the Key Challenges in Murine fMRI" section).

Since anesthesia primarily disrupts neural communication across the brain, its effects are most comprehensively reflected by measures of FC. An ideal sedation for fMRI would meet criteria of a close resemblance of the assessed correlation matrices with those of the awake references, including anticorrelated regions, and a rich repertoire of dynamic FC (see "Functional Connectivity and Murine Resting-State fMRI” section).

\section{MIND Signature}

Another advantage of FC in fMRI is that it can be acquired simply in the form of a resting-state scan without any additional equipment. This makes it a strong candidate measure for determining a MIND signature in translational fMRI. fMRI tasks can be flanked with brief resting-state scans that permit the brain state to be identified at regular points throughout an experiment. Such flanking approaches of FC have previously been shown to reveal valuable information on anesthetic effects on murine fMRI tasks (Nasrallah et al., 2014b; Schroeter et al., 2017; Shim et al., 2018). More advanced FC applications have been used to track spatial and temporal aspects of intermediate brain state transitions as a function of anesthesia in mice (Grandjean et al., 2017a; Gutierrez-Barragan et al., 2018) and rats (Schwalm et al., 2017; Aedo-Jury et al., 2019). Finally, the relations of FC and its structural scaffold have been already used to formulate restingstate dynamics as cortical signatures of anesthesia in monkeys (Barttfeld et al., 2015; Uhrig et al., 2018) and humans (Roberts et al., 2019).

Approaches like these have laid the groundwork for the development of a simple, yet comprehensive signature scheme for the unambiguous identification of anesthetically induced and maintained, intermediate brain states that (1) are adjusted to functionally approximate the awake state and (2) indicate the integrity of a defined set of functional qualifiers. This concept assumes a mechanistic connection between FC and the other qualifiers, so that specific functional properties can be inferred from the detection of a specific MIND signature (Figure 9). Such a mechanistic connection remains to be established-as well as the circumstances in which this assumption can be made with confidence.

\section{Linking the MIND Signature to Other Qualifiers}

Defining a MIND signature that could indicate the cortical state, stimulus-response properties, their hemodynamic translation, and dynamic FC in the form of one unique identifier would be extremely useful. The unambiguous identification of brain states during fMRI would also substantially improve the process of developing (and communicating) novel anesthetic protocols. A verifiable linkage between these qualifiers requires the simultaneous assessment of neural and hemodynamic readouts (see below). However, a large fraction of qualifiers can be assessed in separate measurements. It is reasonable to perform initial anesthetic adjustments outside the MR scanner, based on electrophysiological and behavioral responses to stimuli and cortical signatures during rest (see "Metastability of Brain 
States, Hysteresis, and Behavioral Monitoring" section), while under a tight control of physiological parameters. It is therefore important to select conditions under which the qualifiers measured outside the scanner can be linked to others obtained during fMRI.

Brain states, and thus stimulus response properties, are likely to change during fMRI scans due to the loud gradient noise, which can trigger activity in subcortical arousal nuclei (see "Metastability of Brain States, Hysteresis, and Behavioral Monitoring" section). In fact, fMRI at high levels of sound pressure substantially boosts BOLD responses to subcutaneous electrostimulation of the rat under $\alpha$-chloralose, compared to silent fMRI protocols or after deafening by cochleotomy (Burke et al., 2000). Thus, hearing protection is recommended (Reimann et al., 2016). Sound pressure levels depend on the gradient coils, and can be reduced via sophisticated design of hardware and pulse sequences (Winkler et al., 2018).

Current efforts are underway to advance silent MR techniques using sweep imaging with Fourier transform (SWIFT; Idiyatullin et al., 2006, 2015). This approach renders obsolete the rapid and pulsed on/off switching of strong magnetic field gradients used for spatial encoding, and substantially lowers the acoustic noise level. SWIFT also permits zero echo time TE, which yields distortion- and artifact-free fMRI, even in the face of severe body motion and with electrodes implanted close to the region of interest (Lehto et al., 2018; Paasonen et al., 2020). This is a major advance for the development of sedation protocols to study FC in freely breathing animals and is further attractive for electrophysiological recordings or deep brain stimulation in fMRI. Until SWIFT is readily available for small-bore animal MR scanners, typical sound levels and vibrations produced by the gradients may be mimicked outside the scanner in the electrophysiological setup to test their effects on cortical states. Simultaneous fMRI and EEG (Sadaghiani et al., 2012; Thompson et al., 2013) or $\mathrm{Ca}^{2+}$ imaging (Schwalm et al., 2017; Schlegel et al., 2018) can permit a direct translation between the cortical state and whole-brain hemodynamic responses.

It remains to be clarified to what extent a signature based on FC is sufficient to indicate the integrity of the underlying functional properties of a brain state. Recent studies on the simultaneous imaging of murine cortex-wide FC of hemodynamic and neural calcium fluctuations in the resting state have found a strong relationship between both measurements (Murphy et al., 2017). Both measurements show sufficient resemblance in their intercortical FC for tracking similar brain states under anesthesia (Brier et al., 2019) and similar sets of dynamic FC configurations using a sliding-window approach (Matsui et al., 2018). Transitions in the dynamic FC configurations can be tracked via fMRI within a time scale of 1 or $2 \mathrm{~s}$, and a sliding-window length below $1 \mathrm{~min}$ (Barttfeld et al., 2015, movie S1; Grandjean et al., 2017a; Lurie et al., 2018), which would be sufficient to resolve effects such as the metastable cortical state transitions that were observed via LFP recordings under constant isoflurane concentrations (see "Metastability of Brain States, Hysteresis, and Behavioral Monitoring" sections, Figure 2D; Hudson et al., 2014; Hudson, 2017). However, studies that investigate such brain state transitions under anesthesia linking electrophysiological or calcium recordings with dynamic FC are still pending.

\section{Limitations in Maintaining Stable Brain States}

Even when a brain state is stably maintained over time, it will exhibit a restricted set of recurrent FC configurations that vary within a defined dynamic range (Uhrig et al., 2018). Transitions between two intermediate brain states, as observed for metastable LFP cortical signatures (Hudson et al., 2014), should thus be classifiable as two distinct sets of recurrent FC configurations. Each of these sets varies within the internal dynamic range of its respective brain state. The MIND signature will comprise an indication of the variance of this range (narrow or broad) and the FC patterns that recur (Gutierrez-Barragan et al., 2018). A loss of recurrence and the appearance of new FC configurations would indicate a drift in brain states, and hence a change in anesthetic depth. Transitions in brain states become a confounding factor when they significantly alter the specific functional properties that are relevant for a given fMRI investigation.

The continuous anesthetic infusion via intraperitoneal or subcutaneous administration routes may present a practical experimental challenge because the uptake and distribution of agents depends on various biopharmaceutical and biological factors (comprehensively reviewed in Claassen, 1994). Whether and to what extent it is possible to maintain stable brain states under these conditions has to be established according to specific application procedures (Sirmpilatze et al., 2019). Future technical developments and novel methods should produce increasingly detailed functional information that will need to be integrated into single composite fMRI measurements (Breakspear, 2017; Belloy et al., 2018; Roberts et al., 2019; Song et al., 2019). This will produce a MIND signature that identifies brain states with increasing precision.

\section{The MIND Signature in Translational fMRI}

The MIND signature would be valuable as an indicator of specific brain states independent of minor changes in brain anatomy and thus hold even further value for translational fMRI. It would provide a framework for comparing the MIND signatures of humans and mice and facilitate the development of a scheme for systematic evaluation of functional similarity and divergence across species. This would allow for better translation of anesthetic effects from humans to mice and back, and will have three major benefits: (1) anesthetic effects could be referenced against an awake, but calm and relaxed volunteer-even within the same subject; (2) introspective reports could link specific MIND signatures to LOC and to the overall experience; and (3) determining precise translational limitations in general brain functions and specific anesthetic effects, to assess the scope for murine transgenic and optogenetic applications to explore human cognition and consciousness.

\section{Future Perspectives}

The need for effective anesthetic protocols and a better comprehension of their underpinnings is not exclusive to 
translational fMRI, but is equally important for preclinical researchers studying electrophysiology, $\mathrm{Ca}^{2+}$ imaging, and other modalities to probe brain functions. In clinical practice, balanced multimodal anesthesia is common and increasingly being used as a substitute for opioids, especially in light of the current opioid crisis in the US (Brown et al., 2018a). In basic research, cognitive scientists employ anesthesia to probe the neural correlates of consciousness. All these disparate fields produce valuable data that should be taken into account across research domains in pursuit of a deeper understanding of anesthetic effects on animal and human brain functions.

Yet accessing knowledge on anesthetic effects is not always straightforward. Much valuable information is buried in publications that focus on biological research questions and use anesthesia only as an experimental tool. Many anecdotal observations of crucial effects remain unpublished "lab lore" and are not available to the community. Having access to this information could substantially accelerate our progress in detailing anesthetic effects to develop multimodal protocols for a stable sedation with the best possible preservation of brain functions. A community-driven open database of key observations-collected from publications and lab notes-would make this information more accessible, promoting reproducibility and preventing research groups from running into the same "dead ends" already discovered by others. We are currently developing a web-based infrastructure for such an open initiative across neuroimaging communities, to facilitate the exchange of this information. We invite anyone interested in this initiative to contact the corresponding author.

Within the fMRI community, the additional assessments of resting-state fMRI at selected points throughout the experiment would help to specify the actual brain state and make comparisons of data across labs more reliable. The concept of the MIND signature could support this process by defining a set of qualifiers and one identifier readout that permits a reliable indication of a specific brain state and its related functional properties. Our future work is dedicated to further refining and establishing the MIND signature in a collaborative effort.

\section{CONCLUSIONS}

To make proper use of translational fMRI for a direct, non-invasive comparison between large-scale brain functions in humans and mice, it is essential to understand the

\section{REFERENCES}

Achermann, P., and Borbély, A. A. (1997). Low-frequency $(<1 \mathrm{~Hz})$ oscillations in the human sleep electroencephalogram. Neuroscience 81, 213-222. doi: 10.1016/s0306-4522(97)00186-3

Adamczak, J. M., Farr, T. D., Seehafer, J. U., Kalthoff, D., and Hoehn, M. (2010). High field BOLD response to forepaw stimulation in the mouse. NeuroImage 51, 704-712. doi: 10.1016/j.neuroimage.2010.02.083

Aedo-Jury, F., Schwalm, M., Hamzehpour, L., and Stroh, A. (2019). Brain states govern the spatio-temporal dynamics of resting state functional connectivity. bioRxiv [Preprint]. doi: 10.1101/832998 impact of anesthesia on neural physiology. The animal is usually anesthetized to avoid variations due to stress and distraction, with the ultimate aim of achieving stable sedation while maintaining a functional brain state close to that of an awake, resting subject. Maintaining functional brain states and neurovascular coupling are the two major challenges of preclinical fMRI. Most anesthetics affect neural and hemodynamic integrity in different ways, based on their target receptors, which typically assigns them to one of the three main classes of anesthesia. Despite addressing different pathways, all classes exert their effects by spoiling the phasefrequency relationships of remote neuronal populations, which eventually leads to a breakdown of information transfer along the cortical hierarchy and thus LOC. The resulting disruption of higher-order circuits is reflected in FC, although the specifiability of markers for discrete functional stages remain to be clarified. Better knowledge of the mechanisms of action allows to balance different compounds in order to develop multimodal anesthetic protocols for translational fMRI, ideally revealing mainly species-specific variations and not the influence of anesthesia. The concept of the MIND signature has been proposed as an approach to determine and re-identify specific brain states and related functional properties induced by different anesthetic drugs and levels across studies, laboratories, and species.

\section{AUTHOR CONTRIBUTIONS}

HR: conceiving and drafting the article, agrees to be accountable for all aspects of the work in ensuring that questions related to the accuracy or integrity of any part of the work are appropriately investigated and resolved. TN: revising the article critically, final approval of the submitted version.

\section{ACKNOWLEDGMENTS}

We thank Russ Hodge (Max Delbrück Center for Molecular Medicine, Berlin, Germany), Andreas Pohlmann, Jurjen Heij, Jason Millward (Berlin Ultrahigh Field Facility, Max Delbrück Center for Molecular Medicine, Berlin, Germany), and Nikoloz Sirmpilatze (Functional Imaging Laboratory, Deutsches Primatenzentrum, Göttingen, Germany) for valuable discussions, reading and commenting on the manuscript. 
Akeju, O., Kim, S. E., Vazquez, R., Rhee, J., Pavone, K. J., Hobbs, L. E., et al. (2016a). Spatiotemporal dynamics of dexmedetomidine-induced electroencephalogram oscillations. PLoS One 11:e0163431. doi: 10.1371/journal.pone. 0163431

Akeju, O., Song, A. H., Hamilos, A. E., Pavone, K. J., Flores, F. J., Brown, E. N., et al. (2016b). Electroencephalogram signatures of ketamine anesthesia-induced unconsciousness. Clin. Neurophysiol. 127, 2414-2422. doi: 10.1016/j.clinph. 2016.03.005

Akeju, O., Loggia, M. L., Catana, C., Pavone, K. J., Vazquez, R., Rhee, J., et al. (2014a). Disruption of thalamic functional connectivity is a neural correlate of dexmedetomidine-induced unconsciousness. Elife 3:e04499. doi: 10.7554/eLife. 04499

Akeju, O., Westover, B., Pavone, K. J., Sampson, A. L., Hartnack, K. E., Brown, E. N., et al. (2014b). Effects of sevoflurane and propofol on frontal electroencephalogram power and coherence. Anesthesiology 121, 990-998. doi: $10.1097 / a \ln .0000000000000436$

Aksenov, D. P., Li, L., Miller, M. J., Iordanescu, G., and Wyrwicz, A. M. (2015). Effects of anesthesia on BOLD signal and neuronal activity in the somatosensory cortex. J. Cereb. Blood Flow Metab. 35, 1819-1826. doi: $10.1038 / \mathrm{jcbfm} .2015 .130$

Alheid, G. F., and McCrimmon, D. R. (2008). The chemical neuroanatomy of breathing. Respir. Physiol. Neurobiol. 164, 3-11. doi: 10.1016/j.resp.2008.07.014

Alkire, M. T., Asher, C. D., Franciscus, A. M., and Hahn, E. L. (2009). Thalamic microinfusion of antibody to a voltage-gated potassium channel restores consciousness during anesthesia. Anesthesiology 110, 766-773. doi: 10.1097/aln.0b013e31819c461c

Alkire, M. T., Hudetz, A. G., and Tononi, G. (2008). Consciousness and anesthesia. Science 322, 876-880. doi: 10.1126/science.1149213

Alkire, M. T., and Miller, J. (2005). General anesthesia and the neural correlates of consciousness. Prog. Brain Res. 150, 229-244. doi: 10.1016/s00796123(05)50017-7

Amzica, F. (2009). Basic physiology of burst-suppression. Epilepsia 50, 38-39. doi: 10.1111/j.1528-1167.2009.02345.x

Amzica, F. (2015). What does burst suppression really mean? Epilepsy Behav. 49, 234-237. doi: 10.1016/j.yebeh.2015.06.012

Amzica, F., and Steriade, M. (1997). The K-complex: its slow ( $<1 \mathrm{~Hz})$ rhythmicity and relation to delta waves. Neurology 49, 952-959. doi: 10.1212/wnl.49. 4.952

Anderson, P. M., Jones, N., O'Brien, T. J., and Pinault, D. (2017). The $\mathrm{N}$-Methyl d-Aspartate glutamate receptor antagonist ketamine disrupts the functional state of the corticothalamic pathway. Cereb. Cortex 27, 3172-3185. doi: 10.1093/cercor/bhw168

Andrieu, G., Roth, B., Ousmane, L., Castaner, M., Petillot, P., Vallet, B., et al. (2009). The efficacy of intrathecal morphine with or without clonidine for postoperative analgesia after radical prostatectomy. Anesth. Analg. 108, 1954-1957. doi: 10.1213/ane.0b013e3181a30182

Arena, A., Lamanna, J., Gemma, M., Ripamonti, M., Ravasio, G., Zimarino, V., et al. (2017). Linear transformation of the encoding mechanism for light intensity underlies the paradoxical enhancement of cortical visual responses by sevoflurane. J. Physiol. 595, 321-339. doi: 10.1113/ jp272215

Asanuma, C. (1992). Noradrenergic innervation of the thalamic reticular nucleus: a light and electron microscopic immunohistochemical study in rats. J. Comp. Neurol. 319, 299-311. doi: 10.1002/cne.903190209

Aston-Jones, G., Rajkowski, J., and Cohen, J. (1999). Role of locus coeruleus in attention and behavioral flexibility. Biol. Psychiatry 46, 1309-1320. doi: 10.1016/s0006-3223(99)00140-7

Astori, S., Wimmer, R. D., Prosser, H. M., Corti, C., Corsi, M., Liaudet, N., et al. (2011). The CaV3.3 calcium channel is the major sleep spindle pacemaker in thalamus. Proc. Natl. Acad. Sci. U S A 108, 13823-13828. doi: 10.1073/pnas. 1105115108

Bai, D., Pennefather, P. S., MacDonald, J. F., and Orser, B. A. (1999). The general anesthetic propofol slows deactivation and desensitization of $\mathrm{GABA}_{A}$ receptors. J. Neurosci. 19, 10635-10646. doi: 10.1523/jneurosci.19-24-10 635.1999

Balsters, J. H., Zerbi, V., Sallet, J., Wenderoth, N., and Mars, R. B. (2019). Primate homologs of mouse cortico- striatal circuits. bioRxiv [Preprint]. doi: 10. $1101 / 834481$
Baker, R., Gent, T. C., Yang, Q., Parker, S., Vyssotski, A. L., Wisden, W., et al. (2014). Altered activity in the central medial thalamus precedes changes in the neocortex during transitions into both sleep and propofol anesthesia. J. Neurosci. 34, 13326-13335. doi: 10.1523/JNEUROSCI.1519-14.2014

Bakker, R., Tiesinga, P., and Kötter, R. (2015). The scalable brain atlas: instant web-based access to public brain atlases and related content. Neuroinformatics 13, 353-366. doi: 10.1007/s12021-014-9258-x

Baltes, C., Bosshard, S., Mueggler, T., Ratering, D., and Rudin, M. (2011). Increased blood oxygen level-dependent (BOLD) sensitivity in the mouse somatosensory cortex during electrical forepaw stimulation using a cryogenic radiofrequency probe. NMR Biomed. 24, 439-446. doi: 10.1002/nbm.1613

Banks, M. I., Moran, N. S., Krause, B. M., Grady, S. M., Uhlrich, D. J., and Manning, K. A. (2017). Evidence that loss of consciousness under anesthesia is not associated with impaired stimulus representation in auditory cortex. bioRxiv [Preprint]. doi: 10.1101/213355

Barttfeld, P., Uhrig, L., Sitt, J. D., Sigman, M., Jarraya, B., and Dehaene, S. (2015). Signature of consciousness in the dynamics of resting-state brain activity. Proc. Natl. Acad. Sci. U S A 112, 887-892. doi: 10.1073/pnas.1418031112

Bastos, A. M., Vezoli, J., Bosman, C. A., Schoffelen, J. M., Oostenveld, R., Dowdall, J. R., et al. (2015). Visual areas exert feedforward and feedback influences through distinct frequency channels. Neuron 85, 390-401. doi: 10. 1016/j.neuron.2014.12.018

Baumgart, J. P., Zhou, Z.-Y., Hara, M., Cook, D. C., Hoppa, M. B., Ryan, T. A., et al. (2015). Isoflurane inhibits synaptic vesicle exocytosis through reduced $\mathrm{Ca}^{2+}$ influx, not $\mathrm{Ca}^{2+}$-exocytosis coupling. Proc. Natl. Acad. Sci. U S A 112, 11959-11964. doi: 10.1073/pnas.1500525112

Bazhenov, M., Timofeev, I., Steriade, M., and Sejnowski, T. J. (1999). Self-sustained rhythmic activity in the thalamic reticular nucleus mediated by depolarizing $\mathrm{GABA}_{\mathrm{A}}$ receptor potentials. Nat. Neurosci. 2, 168-174. doi: 10.1038/5729

Beenhakker, M. P., and Huguenard, J. R. (2009). Neurons that fire together also conspire together: is normal sleep circuitry hijacked to generate epilepsy? Neuron 62, 612-632. doi: 10.1016/j.neuron.2009.05.015

Belloy, M. E., Naeyaert, M., Abbas, A., Shah, D., Vanreusel, V., Van Audekerke, J., et al. (2018). Dynamic resting state fMRI analysis in mice reveals a set of Quasi-Periodic Patterns and illustrates their relationship with the global signal. NeuroImage 180, 463-484. doi: 10.1016/j.neuroimage.2018.01.075

Bergmann, E., Zur, G., Bershadsky, G., and Kahn, I. (2016). The organization of mouse and human cortico-hippocampal networks estimated by intrinsic functional connectivity. Cereb. Cortex 26, 4497-4512. doi: 10.1093/cercor/ bhw327

Bertero, A., Liska, A., Pagani, M., Parolisi, R., Masferrer, M. E., Gritti, M., et al. (2018). Autism-associated 16p11.2 microdeletion impairs prefrontal functional connectivity in mouse and human. Brain 141, 2055-2065. doi: 10.1093/brain/ awy111

Bhattacharya, S., Le Cauchois, M. B., Iglesias, P. A., and Chen, Z. S. (2019). Thalamocortical network connectivity controls spatiotemporal dynamics of cortical and thalamic traveling waves. bioRxiv 6:7169. doi: 10.1101/780239

Binda, P., and Gamlin, P. D. (2017). Renewed attention on the pupil light reflex. Trends Neurosci. 40, 455-457. doi: 10.1016/j.tins.2017.06.007

Bittner, S., Gobel, K., and Meuth, S. G. (2019). Cerebral Physiology. Oxford Textbook of Neuroscience and Anaesthesiology. Ann Arbor, MI: Oxford University Press.

Blain-Moraes, S., Lee, U., Ku, S., Noh, G., and Mashour, G. A. (2014). Electroencephalographic effects of ketamine on power, cross-frequency coupling and connectivity in the $\alpha$ bandwidth. Front. Syst. Neurosci. 8:114. doi: 10.3389/fnsys.2014.00114

Blain-Moraes, S., Tarnal, V., Vanini, G., Alexander, A., Rosen, D., Shortal, B., et al. (2015). Neurophysiological correlates of sevoflurane-induced unconsciousness. Anesthesiology 122, 307-316. doi: 10.1097/ALN.0000000000000482

Blazquez Freches, G., Chavarrias, C., and Shemesh, N. (2018). BOLD-fMRI in the mouse auditory pathway. NeuroImage 165, 265-277. doi: 10.1016/j. neuroimage.2017.10.027

Boly, M., Garrido, M. I., Gosseries, O., Bruno, M. A., Boveroux, P., Schnakers, C., et al. (2011). Preserved feedforward but impaired top-down processes in the vegetative state. Science 332, 858-862. doi: 10.1126/science.1202043

Bonhomme, V., Staquet, C., Montupil, J., Defresne, A., Kirsch, M., Martial, C., et al. (2019). General anesthesia: a probe to explore consciousness. Front. Syst. Neurosci. 13:36. doi: 10.3389/fnsys.2019.00036 
Bonhomme, V., Vanhaudenhuyse, A., Demertzi, A., Bruno, M. A., Jaquet, O., Bahri, M. A., et al. (2016). Resting-state network-specific breakdown of functional connectivity during ketamine alteration of consciousness in volunteers. Anesthesiology 125, 873-888. doi: 10.1097/aln.00000000000 01275

Boon, J. A., and Milsom, W. K. (2008). NMDA receptor-mediated processes in the Parabrachial/Kolliker fuse complex influence respiratory responses directly and indirectly via changes in cortical activation state. Respir. Physiol. Neurobiol. 162, 63-72. doi: 10.1016/j.resp.2008.04.002

Börgers, C., Epstein, S., and Kopell, N. J. (2005). Background gamma rhythmicity and attention in cortical local circuits: a computational study. Proc. Natl. Acad. Sci. U S A 102, 7002-7007. doi: 10.1073/pnas. 0502366102

Bosshard, S. C., Baltes, C., Wyss, M. T., Mueggler, T., Weber, B., and Rudin, M. (2010). Assessment of brain responses to innocuous and noxious electrical forepaw stimulation in mice using BOLD fMRI. Pain 151, 655-663. doi: 10.1016/j.pain.2010.08.025

Bosshard, S. C., Stuker, F., Von Deuster, C., Schroeter, A., and Rudin, M. (2015). BOLD fMRI of C-fiber mediated nociceptive processing in mouse brain in response to thermal stimulation of the forepaws. PLoS One 10:e0126513. doi: 10.1371 /journal.pone. 0126513

Boveroux, P., Vanhaudenhuyse, A., Bruno, M. A., Noirhomme, Q., Lauwick, S., Luxen, A., et al. (2010). Breakdown of within- and between-network resting state functional magnetic resonance imaging connectivity during propofol-induced loss of consciousness. Anesthesiology 113, 1038-1053. doi: 10.1097/ALN.0b013e3181f697f5

Bowery, N. G., Hudson, A. L., and Price, G. W. (1987). GABA $A$ and $\mathrm{GABA}_{B}$ receptor site distribution in the rat central nervous system. Neuroscience 20 , 365-383. doi: 10.1016/0306-4522(87)90098-4

Braun, J., and Mattia, M. (2010). Attractors and noise: twin drivers of decisions and multistability. NeuroImage 52, 740-751. doi: 10.1016/j.neuroimage.2009. 12.126

Breakspear, M. (2017). Dynamic models of large-scale brain activity. Nat. Neurosci. 20, 340-352. doi: 10.1038/nn.4497

Bremer, F. (1935). Cerveau isole et Physiologie du sommeil. C. R. Soc. Biol. 118, 1235-1241.

Brier, L. M., Landsness, E. C., Snyder, A. Z., Wright, P. W., Baxter, G. A., Bauer, A. Q., et al. (2019). Separability of calcium slow waves and functional connectivity during wake, sleep and anesthesia. Neurophotonics 6:035002. doi: 10.1117/1.nph.6.3.035002

Brown, E. N., Lydic, R., and Schiff, N. D. (2010). General anesthesia, sleep and coma. N. Engl. J. Med. 363, 2638-2650. doi: 10.1056/NEJMra0808281

Brown, P. L., Zanos, P., Wang, L., Elmer, G. I., Gould, T. D., and Shepard, P. D. (2018). Isoflurane but not halothane prevents and reverses helpless behavior: a role for eeg burst suppression?. Int. J. Neuropsychopharmacol. 21, 777-785. doi: 10.1093/ijnp/pyy029

Brown, E. N., Pavone, K. J., and Naranjo, M. (2018a). Multimodal general anesthesia: theory and practice. Anesth. Analg. 127, 1246-1258. doi: 10.1213/ane.0000000000003668

Brown, E. N., Purdon, P. L., Akeju, O., and An, J. (2018b). Using EEG markers to make inferences about anaesthetic-induced altered states of arousal. $\mathrm{Br}$. J. Anaesth. 121, 325-327. doi: 10.1016/j.bja.2017.12.034

Brown, E. N., Purdon, P. L., and Van Dort, C. J. (2011). General anesthesia and altered states of arousal: a systems neuroscience analysis. Annu. Rev. Neurosci. 34, 601-628. doi: 10.1146/annurev-neuro-060909-153200

Buckwalter, J. A., Parvizi, J., Morecraft, R. J., and Van Hoesen, G. W. (2008). Thalamic projections to the posteromedial cortex in the macaque. J. Comp. Neurol. 507, 1709-1733. doi: 10.1002/cne.21647

Bukhari, Q., Schroeter, A., Cole, D. M., and Rudin, M. (2017). Resting state fMRI in mice reveals anesthesia specific signatures of brain functional networks and their interactions. Front. Neural Circuits 11:5. doi: 10.3389/fncir.2017.00005

Bullmore, E., and Sporns, O. (2012). The economy of brain network organization. Nat. Rev. Neurosci. 13, 336-349. doi: 10.1038/nrn3214

Burke, M., Schwindt, W., Ludwig, U., Hennig, J., and Hoehn, M. (2000). Facilitation of electric forepaw stimulation-induced somatosensory activation in rats by additional acoustic stimulation: an fMRI investigation. Magn. Reson. Med. 44, 317-321. doi: 10.1002/1522-2594(200008)44:2<317::aid-mrm20>3.0. co;2-r
Busche, M. A., Kekuš, M., Adelsberger, H., Noda, T., Förstl, H., Nelken, I., et al. (2015). Rescue of long-range circuit dysfunction in Alzheimer's disease models. Nat. Neurosci. 18, 1623-1630. doi: 10.1038/nn.4137

Buzsáki, G., and Chrobak, J. J. (1995). Temporal structure in spa- tially organized neuronal ensembles: a role for interneuronal networks. Curr. Opin. Neurobiol. 5, 504-510. doi: 10.1016/0959-4388(95)80012-3

Buzsáki, G., and Moser, E. I. (2013). Memory, navigation and theta rhythm in the hippocampal-entorhinal system. Nat. Neurosci. 16, 130-138. doi: 10.1038/ nn.3304

Campagna, J. A., Miller, K. W., and Forman, S. A. (2003). Mechanisms of actions of inhaled anesthetics. N. Engl. J. Med. 348, 2110-2124. doi: $10.1056 /$ nejmra021261

Cao, R., Li, J., Ning, B., Sun, N., Wang, T., Zuo, Z., et al. (2017). Functional and oxygen-metabolic photoacoustic microscopy of the awake mouse brain. NeuroImage 150, 77-87. doi: 10.1016/j.neuroimage.2017.01.049

Carter, M. E., Yizhar, O., Chikahisa, S., Nguyen, H., Adamantidis, A., Nishino, S., et al. (2010). Tuning arousal with optogenetic modulation of locus coeruleus neurons. Nat. Neurosci. 13, 1526-1533. doi: 10.1038/nn.2682

Casali, A. G., Gosseries, O., Rosanova, M., Boly, M., Sarasso, S., Casali, K. R., et al. (2013). A theoretically based index of consciousness independent of sensory processing and behavior. Sci. Transl. Med. 5:198ra105. doi: 10.1126/scitranslmed.3006294

Casarotto, S., Rosanova, M., Gosseries, O., Boly, M., Massimini, M., and Sarasso, S. (2016). "Exploring the neurophysiological correlates of loss and recovery of consciousness: perturbational complexity," in Brain Function and Responsiveness in Disorders of Consciousness, eds M. M. Monti and W. G. Sannita (Cham: Springer International Publishing), 93-104.

Chamadia, S., Pedemonte, J. C., Hahm, E. Y., Mekonnen, J., Ibala, R., Gitlin, J., et al. (2019). Delta oscillations phase limit neural activity during sevoflurane anesthesia. Commun. Biol. 2:415. doi: 10.1038/s42003-019-0664-3

Chan, R. W., Leong, A. T. L., Ho, L. C., Gao, P. P., Wong, E. C., Dong, C. M., et al. (2017). Low-frequency hippocampal-cortical activity drives brain-wide resting-state functional MRI connectivity. Proc. Natl. Acad. Sci. U S A 114, E6972-E6981. doi: 10.1073/pnas.1703309114

Chen, X., Tong, C., Han, Z., Zhang, K., Bo, B., Feng, Y., et al. (2020). Sensory evoked fMRI paradigms in awake mice. NeuroImage 204:116242. doi: 10.1016/j. neuroimage.2019.116242

Chao, Z. C., Takaura, K., Wang, L., Fujii, N., and Dehaene, S. (2018). Largescale cortical networks for hierarchical prediction and prediction error in the primate brain. Neuron 100, 1252.e3-1266.e3. doi: 10.1016/j.neuron.2018. 10.004

Chauvette, S., Crochet, S., Volgushev, M., and Timofeev, I. (2011). Properties of slow oscillation during slow-wave sleep and anesthesia in cats. J. Neurosci. 31, 14998-15008. doi: 10.1523/JNEUROSCI.2339-11.2011

Chen, Z., Wimmer, R. D., Wilson, M. A., and Halassa, M. M. (2016). Thalamic circuit mechanisms link sensory processing in sleep and attention. Front. Neural Circuits 9:83. doi: 10.3389/fncir.2015.00083

Ching, S., and Brown, E. N. (2014). Modeling the dynamical effects of anesthesia on brain circuits. Curr. Opin. Neurobiol. 25, 116-122. doi: 10.1016/j.conb.2013. 12.011

Ching, S., Cimenser, A., Purdon, P. L., Brown, E. N., and Kopell, N. J. (2010). Thalamocortical model for a propofol-induced $\alpha$-rhythm associated with loss of consciousness. Proc. Natl. Acad. Sci. U S A 107, 22665-22670. doi: 10.1073/pnas.1017069108

Ching, S., Purdon, P. L., Vijayan, S., Kopell, N. J., and Brown, E. N. (2012). A neurophysiological-metabolic model for burst suppression. Proc. Natl. Acad. Sci. US A 109, 3095-3100. doi: 10.1073/pnas.1121461109

Chiu, T. H., Chen, M. J., Yang, Y. R., Yang, J. J., and Tang, F. I. (1995). Action of dexmedetomidine on rat locus coeruleus neurones: intracellular recording in vitro. Eur. J. Pharmacol. 285, 261-268. doi: 10.1016/0014-2999(95) 00417-j

Chuang, K. H., and Nasrallah, F. A. (2017). Functional networks and network perturbations in rodents. NeuroImage 163, 419-436. doi: 10.1016/j. neuroimage.2017.09.038

Cimenser, A., Purdon, P. L., Pierce, E. T., Walsh, J. L., Salazar-Gomez, A. F., Harrell, P. G., et al. (2011). Tracking brain states under general anesthesia by using global coherence analysis. Proc. Natl. Acad. Sci. U S A 108, 8832-8837. doi: $10.1073 /$ pnas. 1017041108 
Claassen, V. (1994). "Neglected factors in pharmacology and neuroscience research: biopharmaceutics, animal characteristics, maintenance, testing conditions," in Techniques in the Behavioral and Neural Sciences, Vol. 12, ed. J. P. Huston (New York, NY: Elsevier).

Compte, A., Sanchez-Vives, M. V., McCormick, D. A., and Wang, X. J. (2003). Cellular and network mechanisms of slow oscillatory activity $(<1 \mathrm{~Hz})$ and wave propagations in a cortical network model. J. Neurophysiol. 89, 2707-2725. doi: $10.1152 /$ jn. 00845.2002

Conner, C. R., Ellmore, T. M., Peters, T. A., DiSano, M. A., and Tandon, N. (2011). Variability of the relationship between electrophysiology and BOLD-fMRI across cortical regions in humans. J. Neurosci. 31, 12855-12865. doi: 10.1523/JNEUROSCI.1457-11.2011

Correa-Sales, C., Rabin, B. C., and Maze, M. (1992). A hypnotic response to dexmedetomidine, an $\alpha 2$ agonist, is mediated in the locus coeruleus in rats. Anesthesiology 76, 948-952. doi: 10.1097/00000542-199206000-00013

Coull, J. T. (1998). Neural correlates of attention and arousal: insights from electrophysiology, functional neuroimaging and psychopharmacology. Prog. Neurobiol. 55, 343-361. doi: 10.1016/s0301-0082(98)00011-2

Coulter, K. L., Perier, F., Radeke, C. M., and Vandenberg, C. A. (1995). Identification and molecular localization of a $\mathrm{pH}$-sensing domain for the inward rectifier potassium channel HIR. Neuron 15, 1157-1168. doi: 10.1016/0896-6273(95)90103-5

Craig, A. D. (2013). Cooling, pain and other feelings from the body in relation to the autonomic nervous system. Handb. Clin. Neurol. 117, 103-109. doi: 10.1016/b978-0-444-53491-0.00009-2

Crandall, S. R., Govindaiah, G., and Cox, C. L. (2010). Low-threshold $\mathrm{Ca}^{2+}$ current amplifies distal dendritic signaling in thalamic reticular neurons. J. Neurosci. 30, 15419-15429. doi: 10.1523/JNEUROSCI.3636-10.2010

Crunelli, V., David, F., Lörincz, M. L., and Hughes, S. W. (2015). The thalamocortical network as a single slow wave-generating unit. Curr. Opin. Neurobiol. 31, 72-80. doi: 10.1016/j.conb.2014.09.001

Crunelli, V., and Hughes, S. W. (2010). The slow $(<1 \mathrm{~Hz})$ rhythm of non-REM sleep: a dialogue between three cardinal oscillators. Nat. Neurosci. 13, 9-17. doi: $10.1038 / \mathrm{nn} .2445$

Cunningham, M. O., Pervouchine, D. D., Racca, C., Kopell, N. J., Davies, C. H., Jones, R. S., et al. (2006). Neuronal metabolism governs cortical network response state. Proc. Natl. Acad. Sci. U S A 103, 5597-5601. doi: 10.1073/pnas. 0600604103

Dagal, A., and Lam, A. M. (2009). Cerebral autoregulation and anesthesia. Curr. Opin. Anaesthesiol. 22, 547-552. doi: 10.1097/ACO.0b013e32833020be

Dang-Vu, T. T., McKinney, S. M., Buxton, O. M., Solet, J. M., and Ellenbogen, J. M. (2010). Spontaneous brain rhythms predict sleep stability in the face of noise. Curr. Biol. 20, R626-R627. doi: 10.1016/j.cub.2010.06.032

Davis, K. D., Flor, H., Greely, H. T., Iannetti, G. D., Mackey, S., Ploner, M., et al. (2017). Brain imaging tests for chronic pain: medical, legal and ethical issues and recommendations. Nat Rev Neurol 13, 624-638. doi: 10.1038/nrneurol. 2017.122

de la Salle, S., Choueiry, J., Shah, D., Bowers, H., McIntosh, J., Ilivitsky, V., et al. (2016). Effects of ketamine on resting-state EEG activity and their relationship to perceptual/dissociative symptoms in healthy humans. Front. Pharmacol. 7, 348-348. doi: 10.3389/fphar.2016.00348

Deleuze, C., and Huguenard, J. R. (2016). Two classes of excitatory synaptic responses in rat thalamic reticular neurons. J. Neurophysiol. 116, 995-1011. doi: $10.1152 /$ jn. 01121.2015

Desai, M., Kahn, I., Knoblich, U., Bernstein, J., Atallah, H., Yang, A., et al. (2011). Mapping brain networks in awake mice using combined optical neural control and fMRI. J. Neurophysiol. 105, 1393-1405. doi: 10.1152/jn. 00828.2010

Destexhe, A., and Contreras, D. (2006). Neuronal computations with stochastic network states. Science 314, 85-90. doi: 10.1126/science.1127241

Destexhe, A., Contreras, D., Sejnowski, T. J., and Steriade, M. (1994). A model of spindle rhythmicity in the isolated thalamic reticular nucleus. J. Neurophysiol. 72, 803-818. doi: 10.1152/jn.1994.72.2.803

Destexhe, A., Contreras, D., Steriade, M., Sejnowski, T. J., and Huguenard, J. R. (1996). In vivo, in vitro, and computational analysis of dendritic calcium currents in thalamic reticular neurons. J. Neurosci. 16, 169-185. doi: 10.1523/JNEUROSCI.16-01-00169.1996
Destexhe, A., Hughes, S. W., Rudolph, M., and Crunelli, V. (2007). Are corticothalamic 'up' states fragments of wakefulness? Trends Neurosci. 30, 334-342. doi: 10.1016/j.tins.2007.04.006

Detsch, O., Kochs, E., Siemers, M., Bromm, B., and Vahle-Hinz, C. (2002). Increased responsiveness of cortical neurons in contrast to thalamic neurons during isoflurane-induced EEG bursts in rats. Neurosci. Lett. 317, 9-12. doi: 10.1016/s0304-3940(01)02419-3

Detsch, O., Vahle-Hinz, C., Kochs, E., Siemers, M., and Bromm, B. (1999). Isoflurane induces dose-dependent changes of thalamic somatosensory information transfer. Brain Res. 829, 77-89. doi: 10.1016/s00068993(99)01341-4

Devonshire, I. M., Grandy, T. H., Dommett, E. J., and Greenfield, S. A. (2010). Effects of urethane anaesthesia on sensory processing in the rat barrel cortex revealed by combined optical imaging and electrophysiology. Eur. J. Neurosci. 32, 786-797. doi: 10.1111/j.1460-9568.2010.07322.x

Dopfel, D., and Zhang, N. (2018). Mapping stress networks using functional magnetic resonance imaging in awake animals. Neurobiol. Stress 9, 251-263. doi: 10.1016/j.ynstr.2018.06.002

D'Souza, R. D., Meier, A. M., Bista, P., Wang, Q. and Burkhalter, A. (2016). Recruitment of inhibition and excitation across mouse visual cortex depends on the hierarchy of interconnecting areas. Elife 5:e19332. doi: 10.7554/eLife. 19332

Duling, B. R., and Berne, R. M. (1970). Longitudinal gradients in periarteriolar oxygen tension. A possible mechanism for the participation of oxygen in local regulation of blood flow. Circ. Res. 27, 669-678. doi: 10.1161/01.res.27. 5.669

Echlin, F. A., Arnett, V., and Zoll, J. (1952). Paroxysmal high voltage discharges from isolated and partially isolated human and animal cerebral cortex. Electroencephalogr. Clin. Neurophysiol. 4, 147-164. doi: 10.1016/00134694(52)90004-7

Ecker, A. S., Denfield, G. H., Bethge, M., and Tolias, A. S. (2016). On the structure of neuronal population activity under fluctuations in attentional state. J. Neurosci. 36, 1775-1789. doi: 10.1523/JNEUROSCI.2044-15.2016

Eger, E. I. II., Saidman, L. J., and Brandstater, B. (1965). Minimum alveolar anesthetic concentration: a standard of anesthetic potency. Anesthesiology 26, 756-763. doi: 10.1097/00000542-196511000-00010

Erisken, S., Vaiceliunaite, A., Jurjut, O., Fiorini, M., Katzner, S., and Busse, L. (2014). Effects of locomotion extend throughout the mouse early visual system. Curr. Biol. 24, 2899-2907. doi: 10.1016/j.cub.2014.10.045

Erö, C., Gewaltig, M.-O., Keller, D., and Markram, H. (2018). A cell atlas for the mouse brain. Front. Neuroinform. 12:84. doi: 10.3389/fninf.2018.00084

Eschenko, O., Mölle, M., Born, J., and Sara, S. J. (2006). Elevated sleep spindle density after learning or after retrieval in rats. J. Neurosci. 26, 12914-12920. doi: 10.1523/JNEUROSCI.3175-06.2006

Fernandez, L. M. J., Pellegrini, C., Vantomme, G., Béard, E., Lüthi, A., and Astori, S. (2017). Cortical afferents onto the nucleus Reticularis thalami promote plasticity of low-threshold excitability through GluN2C-NMDARs. Sci. Rep. 7:12271. doi: 10.1038/s41598-017-12552-8

Ferrarelli, F., Massimini, M., Sarasso, S., Casali, A., Riedner, B. A., Angelini, G., et al. (2010). Breakdown in cortical effective connectivity during midazolaminduced loss of consciousness. Proc. Natl. Acad. Sci. U S A 107, 2681-2686. doi: 10.1073/pnas.0913008107

Ferrari, L., Turrini, G., Crestan, V., Bertani, S., Cristofori, P., Bifone, A., et al. (2012). A robust experimental protocol for pharmacological fMRI in rats and mice. J. Neurosci. Methods 204, 9-18. doi: 10.1016/j.jneumeth.2011. 10.020

Ferron, J.-F., Kroeger, D., Chever, O., and Amzica, F. (2009). Cortical inhibition during burst suppression induced with isoflurane anesthesia. J. Neurosci. 29, 9850-9860. doi: 10.1523/JNEUROSCI.5176-08.2009

Flores, F. J., Hartnack, K. E., Fath, A. B., Kim, S.-E., Wilson, M. A., Brown, E. N., et al. (2017). Thalamocortical synchronization during induction and emergence from propofol-induced unconsciousness. Proc. Natl. Acad. Sci. U S A 114, E6660-E6668. doi: 10.1073/pnas.1700148114

Franceschini, M. A., Radhakrishnan, H., Thakur, K., Wu, W., Ruvinskaya, S., Carp, S., et al. (2010). The effect of different anesthetics on neurovascular coupling. NeuroImage 51, 1367-1377. doi: 10.1016/j.neuroimage. 2010.03.060 
Frankish, A., Diekhans, M., Ferreira, A. M., Johnson, R., Jungreis, I., Loveland, J., et al. (2019). GENCODE reference annotation for the human and mouse genomes. Nucleic Acids Res. 47, D766-D773. doi: 10.1093/nar/gky955

Franks, N. P. (2008). General anaesthesia: from molecular targets to neuronal pathways of sleep and arousal. Nat. Rev. Neurosci. 9, 370-386. doi: $10.1038 / \mathrm{nrn} 2372$

Friedman, E. B., Sun, Y., Moore, J. T., Hung, H. T., Meng, Q. C., Perera, P., et al. (2010). A conserved behavioral state barrier impedes transitions between anesthetic-induced unconsciousness and wakefulness: evidence for neural inertia. PLoS One 5:e11903. doi: 10.1371/journal.pone.0011903

Fries, P. (2015). Rhythms for cognition: communication through coherence. Neuron 88, 220-235. doi: 10.1016/j.neuron.2015.09.034

Fries, P., Nikolić, D., and Singer, W. (2007). The $\gamma$ cycle. Trends Neurosci. 30, 309-316. doi: 10.1016/j.tins.2007.05.005

Fu, B., Yu, T., Yuan, J., Gong, X., and Zhang, M. (2017). Noradrenergic transmission in the central medial thalamic nucleus modulates the electroencephalographic activity and emergence from propofol anesthesia in rats. J. Neurochem. 140, 862-873. doi: 10.1111/jnc.13939

Fuentealba, P., and Steriade, M. (2005). The reticular nucleus revisited: intrinsic and network properties of a thalamic pacemaker. Prog. Neurobiol. 75, 125-141. doi: 10.1016/j.pneurobio.2005.01.002

Fukuda, M., Vazquez, A. L., Zong, X., and Kim, S. G. (2013). Effects of the $\alpha_{2}$-adrenergic receptor agonist dexmedetomidine on neural, vascular and BOLD fMRI responses in the somatosensory cortex. Eur. J. Neurosci. 37, 80-95. doi: 10.1111/ejn.12024

Fulcher, B. D., and Fornito, A. (2016). A transcriptional signature of hub connectivity in the mouse connectome. Proc. Natl. Acad. Sci. U S A 113, 1435-1440. doi: 10.1073/pnas.1513302113

Fulcher, B. D., Murray, J. D., Zerbi, V., and Wang, X.-J. (2019). Multimodal gradients across mouse cortex. Proc. Natl. Acad. Sci. U S A 116, 4689-4695. doi: $10.1073 /$ pnas. 1814144116

Fuller, P. M., Sherman, D., Pedersen, N. P., Saper, C. B., and Lu, J. (2011). Reassessment of the structural basis of the ascending arousal system. J. Comp. Neurol. 519, 933-956. doi: 10.1002/cne.22559

Furth, K. E., McCoy, A. J., Dodge, C., Walters, J. R., Buonanno, A., and Delaville, C. (2017). Neuronal correlates of ketamine and walking induced $\gamma$ oscillations in the medial prefrontal cortex and mediodorsal thalamus. PLoS One 12:e186732. doi: 10.1371/journal.pone.0186732

Gămănuț, R., Kennedy, H., Toroczkai, Z., Ercsey-Ravasz, M., Van Essen, D. C., Knoblauch, K., et al. (2018). The mouse cortical connectome, characterized by an ultra-dense cortical graph, maintains specificity by distinct connectivity profiles. Neuron 97, 698.e10-715.e10. doi: 10.1016/j.neuron.2017. 12.037

Giorgi, A., Migliarini, S., Galbusera, A., Maddaloni, G., Mereu, M., Margiani, G., et al. (2017). Brainwide mapping of endogenous serotonergic transmission via chemogenetic-fMRI. Cell Rep. 21, 910-918. doi: 10.1016/j.celrep.2017.09.087

Glickfeld, L. L., and Olsen, S. R. (2017). Higher-order areas of the mouse visual cortex. Annu. Rev. Vis. Sci. 3, 251-273. doi: 10.1146/annurev-vision-102016061331

Golkowski, D., Ranft, A., Kiel, T., Riedl, V., Kohl, P., Rohrer, G., et al. (2017). Coherence of BOLD signal and electrical activity in the human brain during deep sevoflurane anesthesia. Brain Behav. 7:e00679. doi: 10.1002/brb3.679

Golkowski, D., Larroque, S. K., Vanhaudenhuyse, A., Plenevaux, A., Boly, M., Di Perri, C., et al. (2019). Changes in whole brain dynamics and connectivity patterns during sevoflurane-and propofol-induced unconsciousness identified by functional magnetic resonance imaging. Anesthesiology 130, 898-911. doi: 10.1097/aln.0000000000002704

Gozzi, A., Ceolin, L., Schwarz, A., Reese, T., Bertani, S., Crestan, V., et al. (2007). A multimodality investigation of cerebral hemodynamics and autoregulation in pharmacological MRI. Magn. Reson. Imaging 25, 826-833. doi: 10.1016/j.mri. 2007.03.003

Gozzi, A., and Schwarz, A. J. (2016). Large-scale functional connectivity networks in the rodent brain. NeuroImage 127, 496-509. doi: 10.1016/j.neuroimage.2015. 12.017

Grandjean, J., Canella, C., Anckaerts, C., Ayranc1, G., Bougacha, S., Bienert, T., et al. (2020). Common functional networks in the mouse brain revealed by multi-centre resting-state fMRI analysis. NeuroImage 116278. doi: 10.1016/j.neuroimage.2019.116278
Grandjean, J., Canella, C., Anckaerts, C., Ayrancı, G., Bougacha, S., Bienert, T., et al. (2019a). Common functional networks in the mouse brain revealed by multi-centre resting-state fMRI analysis. bioRxiv [Preprint]

Grandjean, J., Corcoba, A., Kahn, M. C., Upton, A. L., Deneris, E. S., Seifritz, E., et al. (2019b). A brain-wide functional map of the serotonergic responses to acute stress and fluoxetine. Nat. Commun. 10:350. doi: 10.1038/s41467-01808256-w

Grandjean, J., Preti, M. G., Bolton, T. A. W., Buerge, M., Seifritz, E., and Pryce, C. R. et al. (2017a). Dynamic reorganization of intrinsic functional networks inthe mouse brain. NeuroImage 152, 497-508. doi: 10.1016/j.neuroimage.2017.03.026

Grandjean, J., Zerbi, V., Balsters, J. H., Wenderoth, N., and Rudin, M. (2017b). Structural basis of large-scale functional connectivity in the mouse. J. Neurosci. 37, 8092-8101. doi: 10.1523/JNEUROSCI.0438-17.2017

Grandjean, J., Schroeter, A., Batata, I., and Rudin, M. (2014). Optimization of anesthesia protocol for resting-state fMRI in mice based on differential effects of anesthetics on functional connectivity patterns. NeuroImage 102, 838-847. doi: 10.1016/j.neuroimage.2014.08.043

Gray, C. M., and McCormick, D. A. (1996). Chattering cells: superficial pyramidal neurons contributing to the generation of synchronous oscillations in the visual cortex. Science 274, 109-113. doi: 10.1126/science.274.5284.109

Greenberg, D. S., Houweling, A. R., and Kerr, J. N. (2008). Population imaging of ongoing neuronal activity in the visual cortex of awake rats. Nat. Neurosci. 11, 749-751. doi: 10.1038/nn.2140

Grunze, H. C. R., Rainnie, D. G., Hasselmo, M. E., Barkai, E., Hearn, E. F., McCarley, R. W., et al. (1996). NMDA-dependent modulation of CA1 local circuit inhibition. J. Neurosci. 16, 2034-2043. doi: 10.1523/JNEUROSCI.16-0602034.1996

Guidera, J. A., Taylor, N. E., Lee, J. T., Vlasov, K. Y., Pei, J., Stephen, E. P., et al. (2017). Sevoflurane induces coherent slow-delta oscillations in rats. Front. Neural Circuits 11:36. doi: 10.3389/fncir.2017.00036

Guilfoyle, D. N., Gerum, S. V., Sanchez, J. L., Balla, A., Sershen, H., Javitt, D. C., et al. (2013). Functional connectivity fMRI in mouse brain at $7 \mathrm{~T}$ using isoflurane. J. Neurosci. Methods 214, 144-148. doi: 10.1016/j.jneumeth.2013. 01.019

Guldenmund, P., Vanhaudenhuyse, A., Sanders, R., Sleigh, J., Bruno, M. A., Demertzi, A., et al. (2017). Brainfunctional connectivity differentiates dexmedetomidinefrom propofol and natural sleep. Br. J. Anaesth. 119, 674-684. doi: 10.1093/bja/aex257

Gunduz-Bruce, H. (2009). The acute effects of NMDA antagonism: from the rodent to the human brain. Brain Res. Rev. 60, 279-286. doi: 10.1016/j. brainresrev.2008.07.006

Gutierrez-Barragan, D., Basson, M. A., Panzeri, S., and Gozzi, A. (2018). Oscillatory brain states govern spontaneous fMRI network dynamics. bioRxiv [Preprint]. doi: 10.1101/393389

Hahn, T. T. G., Sakmann, B., and Mehta, M. R. (2006). Phase-locking of hippocampal interneurons' membrane potential to neocortical up-down states. Nat. Neurosci. 9, 1359-1361. doi: 10.1038/nn1788

Haider, B., Häusser, M., and Carandini, M. (2013). Inhibition dominates sensory responses in the awake cortex. Nature 493, 97-100. doi: 10.1038/ nature 11665

Halassa, M. M., Siegle, J. H., Ritt, J. T., Ting, J. T., Feng, G., and Moore, C. I. (2011). Selective optical drive of thalamic reticular nucleus generates thalamic bursts and cortical spindles. Nat. Neurosci. 14, 1118-1120. doi: 10.1038/nn. 2880

Hall, C. N., Reynell, C., Gesslein, B., Hamilton, N. B., Mishra, A., Sutherland, B. A., et al. (2014). Capillary pericytes regulate cerebral blood flow in health and disease. Nature 508, 55-60. doi: 10.1038/nature13165

Halley, A. C., and Krubitzer, L. (2019). Not all cortical expansions are the same: the coevolution of the neocortex and the dorsal thalamus in mammals. Curr. Opin. Neurobiol. 56, 78-86. doi: 10.1016/j.conb.2018.12.003

Hamilton, N. B., Attwell, D., and Hall, C. N. (2010). Pericyte-mediated regulation of capillary diameter: a component of neurovascular coupling in health and disease. Front. Neuroenergetics 2:5. doi: 10.3389/fnene. 2010.00005

Hamner, J. W., Ishibashi, K., and Tan, C. O. (2019). Revisiting human cerebral blood flow responses to augmented blood pressure oscillations. J. Physiol. 597, 1553-1564. doi: 10.1113/jp277321 
Han, Z., Chen, W., Chen, X., Zhang, K., Tong, C., Zhang, X., et al. (2019). Awake and behaving mouse fMRI during Go/No-Go task. NeuroImage 188, 733-742. doi: 10.1016/j.neuroimage.2019.01.002

Han, H.-B., Lee, K. E., and Choi, J. H. (2019). Functional dissociation of $\theta$ oscillations in the frontal and visual cortices and their long-range network during sustained attention. eNeuro 6:ENEURO.0248-19.2019. doi: 10 . 1523/ENEURO.0248-19.2019

Harris, K. D., and Thiele, A. (2011). Cortical state and attention. Nat. Rev. Neurosci. 12, 509-523. doi: 10.1038/nrn3084

Hartikainen, K. M., Rorarius, M., Peräkylä, J. J., Laippala, P. J., and Jäntti, V. (1995). Cortical reactivity during isoflurane burst-suppression anesthesia. Anesth. Analg. 81, 1223-1228. doi: 10.1213/00000539-199512000-00018

Havlik, M. (2017). Missing piece of the puzzle in the science of consciousness: resting state and endogenous correlates of consciousness. Conscious. Cogn. 49, 70-85. doi: 10.1016/j.concog.2017.01.006

Hayat, H., Regev, N., Matosevich, N., Sales, A., Paredes-Rodriguez, E., Krom, A. J., et al. (2019). Locus-coeruleus norepinephrine activity gates sensory-evoked awakenings from sleep. bioRxiv [Preprint]. doi: 10.1101/539502

Hayton, S. M., Kriss, A., and Muller, D. P. (1999). Comparison of the effects of four anaesthetic agents on somatosensory evoked potentials in the rat. Lab. Anim. 33, 243-251. doi: 10.1258/002367799780578219

Heindl-Erdmann, C., Axmann, R., Kreitz, S., Zwerina, J., Penninger, J., Schett, G., et al. (2010). Combining functional magnetic resonance imaging with mouse genomics: new options in pain research. Neuroreport 21, 29-33. doi: 10.1097/wnr.0b013e3283324faf

Hemmings, H. C. Jr., Akabas, M. H., Goldstein, P. A., Trudell, J. R., Orser, B. A., and Harrison, N. L. (2005). Emerging molecular mechanisms of general anesthetic action. Trends Pharmacol. Sci. 26, 503-510. doi: 10.1016/j.tips.2005. 08.006

Hemmings, H. C. Jr., Riegelhaupt, P. M., Kelz, M. B., Solt, K., Eckenhoff, R. G., Orser, B. A., et al. (2019). Towards a comprehensive understanding of anesthetic mechanisms of action: a decade of discovery. Trends Pharmacol. Sci. 40, 464-481. doi: 10.1016/j.tips.2019.05.001

Henry, C. E., and Scoville, W. B. (1952). Suppression-burst activity from isolated cerebral cortex in man. Electroencephalogr. Clin. Neurophysiol. 4, 1-22. doi: 10.1016/0013-4694(52)90027-8

Hentschke, H., Raz, A., Krause, B. M., Murphy, C. A., and Banks, M. I. (2017). Disruption of cortical network activity by the general anaesthetic isoflurane. Br. J. Anaesth. 119, 685-696. doi: 10.1093/bja/aex199

Herrera, C. G., Cadavieco, M. C., Jego, S., Ponomarenko, A., Korotkova, T., and Adamantidis, A. (2016). Hypothalamic feedforward inhibition of thalamocortical network controls arousal and consciousness. Nat. Neurosci. 19, 290-298. doi: 10.1038/nn.4209

Hess, A., Axmann, R., Rech, J., Finzel, S., Heindl, C., Kreitz, S., et al. (2011). From the cover: blockade of TNF- $\alpha$ rapidly inhibits pain responses in the central nervous system. Proc. Natl. Acad. Sci. US A 108, 3731-3736. doi: 10.1073/pnas. 1011774108

Hirsch, N., and Taylor, C. (2010). Pharmacological and pathological modulation of cerebral physiology. Anaesth. Intens. Care Med. 11, 349-354. doi: 10.1016/j. mpaic.2010.05.011

Hofman, M. A. (2014). Evolution of the human brain: when bigger is better. Front. Neuroanat. 8:15. doi: 10.3389/fnana.2014.00015

Homayoun, H., and Moghaddam, B. (2007). NMDA receptor hypofunction produces opposite effects on prefrontal cortex interneurons and pyramidal neurons. J. Neurosci. 27, 11496-11500. doi: 10.1523/JNEUROSCI. 2213-07.2007

Hoover, W. B., and Vertes, R. P. (2007). Anatomical analysis of afferent projections to the medial prefrontal cortex in the rat. Brain Struct. Funct. 212, 149-179. doi: 10.1007/s00429-007-0150-4

Hopfield, J. J. (1995). Pattern recognition computation using action potential timing for stimulus representation. Nature 376, 33-36. doi: 10.1038/376033a0

Horovitz, S. G., Fukunaga, M., de Zwart, J. A., van Gelderen, P., Fulton, S. C., Balkin, T. J., et al. (2008). Low frequency BOLD fluctuations during resting wakefulness and light sleep: a simultaneous EEG-fMRI study. Hum. Brain Mapp. 29, 671-682. doi: 10.1002/hbm.20428

Hoyer, C., Gass, N., Weber-Fahr, W., and Sartorius, A. (2014). Advantages and challenges of small animal magnetic resonance imaging as a translational tool. Neuropsychobiology 69, 187-201. doi: 10.1159/000360859
Huang, W., Plyka, I., Li, H., Eisenstein, E. M., Volkow, N. D., and Springer, C. S. Jr. (1996). Magnetic resonance imaging (MRI) detection of the murine brain response to light: temporal differentiation and negative functional MRI changes. Proc. Natl. Acad. Sci. U S A 93, 6037-6042. doi: 10.1073/pnas.93. 12.6037

Huang, Z., Wang, Z., Zhang, J., Dai, R., Wu, J., Li, Y., et al. (2014). Altered temporal variance and neural synchronization of spontaneous brain activity in anesthesia. Hum. Brain Mapp. 35, 5368-5378. doi: 10.1002/hbm.22556

Huber, L., Handwerker, D. A., Jangraw, D. C., Chen, G., Hall, A., Stüber, C., et al. (2017). High-resolution CBV-fMRI allows mapping of laminar activity and connectivity of cortical input and output in human M1. Neuron 96, 1253.e7-1263.e7. doi: 10.1016/j.neuron.2017.11.005

Hudetz, A. G. (2002). Effect of volatile anesthetics on interhemispheric EEG crossapproximate entropy in the rat. Brain Res. 954, 123-131. doi: 10.1016/s00068993(02)03358-9

Hudetz, A. G., and Imas, O. A. (2007). Burst activation of the cerebral cortex by flash stimuli during isoflurane anesthesia in rats. Anesthesiology 107, 983-991. doi: 10.1097/01.anes.0000291471.80659.55

Hudetz, A. G., and Mashour, G. A. (2016). Disconnecting consciousness: is there a common anesthetic end point? Anesth. Analg. 123, 1228-1240. doi: 10.1213/ane.0000000000001353

Hudetz, A. G., Wood, J. D., and Kampine, J. P. (2003). Cholinergic reversal of isoflurane anesthesia in rats as measured by cross-approximate entropy of the electroencephalogram. Anesthesiology 99, 1125-1131. doi: 10.1097/00000542200311000-00019

Hudson, A. E. (2017). Metastability of neuronal dynamics during general anesthesia: time for a change in our assumptions? Front. Neural Circuits 11:58. doi: 10.3389/fncir.2017.00058

Hudson, A. E., Calderon, D. P., Pfaff, D. W., and Proekt, A. (2014). Recovery of consciousness is mediated by a network of discrete metastable activity states. Proc. Natl. Acad. Sci. U S A 111, 9283-9288. doi: 10.1073/pnas.1408296111

Hughes, R. J. (1986). Extreme stereotypy in the burst suppression pattern. Clin. Electroencephalogr. 17, 162-168.

Hutchison, R. M., Hutchison, M., Manning, K. Y., Menon, R. S., and Everling, S. (2014). Isoflurane induces dose-dependent alterations in the cortical connectivity profiles and dynamic properties of the brain's functional architecture. Hum. Brain Mapp. 35, 5754-5775. doi: 10.1002/hbm. 22583

Huupponen, E., Maksimow, A., Lapinlampi, P., Särkelä, M., Saastamoinen, A., Snapir, A., et al. (2008). Electroencephalogram spindle activity during dexmedetomidine sedation and physiological sleep. Acta Anaesthesiol. Scand. 52, 289-294. doi: 10.1111/j.1399-6576.2007.01537.x

Idiyatullin, D., Corum, C. A., and Garwood, M. (2015). Multi-band-SWIFT. J. Magn. Reson. 251, 19-25. doi: 10.1016/j.jmr.2014.11.014

Idiyatullin, D., Corum, C., Park, J. Y., and Garwood, M. (2006). Fast and quiet MRI using a swept radiofrequency. J. Magn. Reson. 181, 342-349. doi: 10.1016/j.jmr. 2006.05.014

Iida, H., Ohata, H., Iida, M., Watanabe, Y., and Dohi, S. (1998). Isoflurane and sevoflurane induce vasodilation of cerebral vessels via ATP-sensitive $\mathrm{K}^{+}$channel activation. Anesthesiology 89, 954-960. doi: 10.1097/00000542199810000-00020

Imas, O. A., Ropella, K. M., Ward, B. D., Wood, J. D., and Hudetz, A. G. (2005a). Volatile anesthetics enhance flash-induced $\gamma$ oscillations in rat visual cortex. Anesthesiology 102, 937-947. doi: 10.1097/00000542-20050500000012

Imas, O. A., Ropella, K. M., Ward, B. D., Wood, J. D., and Hudetz, A. G. (2005b). Volatile anesthetics disrupt frontal-posterior recurrent information transfer at gamma frequencies in rat. Neurosci. Lett. 387, 145-150. doi: 10.1016/j.neulet. 2005.06.018

Isaacson, J. S., and Scanziani, M. (2011). How inhibition shapes cortical activity. Neuron 72, 231-243. doi: 10.1016/j.neuron.2011.09.027

Jensen, O., Spaak, E., and Zumer, J. M. (2019). "Human brain oscillations: from physiological mechanisms to analysis and cognition," in Magnetoencephalography, eds S. Supek and C. Aine (Cham: Springer), $1-46$.

Ji, D., and Wilson, M. A. (2007). Coordinated memory replay in the visual cortex and hippocampus during sleep. Nat. Neurosci. 10, 100-107. doi: $10.1038 / \mathrm{nn} 1825$ 
Jonckers, E., Delgado Y Palacios, R., Shah, D., Guglielmetti, C., Verhoye, M., and Van der Linden, A. (2014). Different anesthesia regimes modulate the functional connectivity outcome in mice. Magn. Reson. Med. 72, 1103-1112. doi: $10.1002 / \mathrm{mrm} .24990$

Jonckers, E., Shah, D., Hamaide, J., Verhoye, M., and Van der Linden, A. (2015). The power of using functional fMRI on small rodents to study brain pharmacology and disease. Front. Pharmacol. 6:231. doi: 10.3389/fphar.2015. 00231

Jonckers, E., Van Audekerke, J., De Visscher, G., Van der Linden, A., and Verhoye, M. (2011). Functional connectivity fMRI of the rodent brain: comparison of functional connectivity networks in rat and mouse. PLoS One 6:e18876. doi: 10.1371/journal.pone.0018876

Jones, M., Berwick, J., Hewson-Stoate, N., Gias, C., and Mayhew, J. (2005). The effect of hypercapnia on the neural and hemodynamic responses to somatosensory stimulation. NeuroImage 27, 609-623. doi: 10.1016/j. neuroimage.2005.04.036

Jones, B. E., and Yang, T. Z. (1985). The efferent projections from the reticular formation and the locus coeruleus studied by anterograde and retrograde axonal transport in the rat. J. Comp. Neurol. 242, 56-92. doi: 10.1002/cne. 902420105

Jordan, D., Ilg, R., Riedl, V., Schorer, A., Grimberg, S., Neufang, S., et al. (2013). Simultaneous electroencephalographic and functional magnetic resonance imaging indicate impaired cortical top-down processing in association with anesthetic-induced unconsciousness. Anesthesiology 119, 1031-1042. doi: 10.3410/f.718082755.793486169

Jorm, C. M., and Stamford, J. A. (1993). Actions of the hypnotic anaesthetic, dexmedetomidine, on noradrenaline release and cell firing in rat locus coeruleus slices. Br. J. Anaesth. 71, 447-449. doi: 10.1093/bja/ 71.3.447

Joshi, S., Li, Y., Kalwani, R. M., and Gold, J. I. (2016). Relationships between pupil diameter and neuronal activity in the locus coeruleus, colliculi, and cingulate cortex. Neuron 89, 221-234. doi: 10.1016/j.neuron.2015. 11.028

Jung, W. B., Shim, H. J., and Kim, S. G. (2019). Mouse BOLD fMRI at ultrahigh field detects somatosensory networks including thalamic nuclei. NeuroImage 195, 203-214. doi: 10.1016/j.neuroimage.2019.03.063

Kahn, I., Desai, M., Knoblich, U., Bernstein, J., Henninger, M., Graybiel, A. M., et al. (2011). Characterization of the functional MRI response temporal linearity via optical control of neocortical pyramidal neurons. J. Neurosci. 31, 15086-15091. doi: 10.1523/JNEUROSCI.0007-11.2011

Kalisch, R., Elbel, G.-K., Gössl, C., Czisch, M., and Auer, D. P. (2001). Blood pressure changes induced by arterial blood withdrawal influence bold signal in anesthesized rats at 7 tesla: implications for pharmacologic MRI. NeuroImage 14, 891-898. doi: 10.1006/nimg.2001.0890

Kalthoff, D., Seehafer, J. U., Po, C., Wiedermann, D., and Hoehn, M. (2011). Functional connectivity in the rat at $11.7 \mathrm{~T}$ : impact of physiological noise in resting state fMRI. NeuroImage 54, 2828-2839. doi: 10.1016/j.neuroimage. 2010.10.053

Kamibayashi, T., and Maze, M. (2000). Clinical uses of $\alpha-2$ adrenergic agonists. Anesthesiology 93, 1345-1349. doi: 10.1097/00000542-200011000-00030

Kanai, R., Komura, Y., Shipp, S., and Friston, K. (2015). Cerebral hierarchies: predictive processing, precision and the pulvinar. Philos. Trans. R. Soc. Lond. B Biol. Sci. 370:20140169. doi: 10.1098/rstb.2014.0169

Kandel, A., and Buzsáki, G. (1997). Cellular-synaptic generation of sleep spindles, spike-and-wave discharges, and evoked thalamocortical responses in the neocortex of the rat. J. Neurosci. 17, 6783-6797. doi: 10.1523/JNEUROSCI.1717-06783.1997

Kastner, S., and Ungerleider, L. G. (2000). Mechanisms of visual attention in the human cortex. Annu. Rev. Neurosci. 23, 315-341. doi: 10.1146/annurev.neuro. 23.1.315

Kayser, C., Montemurro, M. A., Logothetis, N. K., and Panzeri, S. (2009). Spike-phase coding boosts and stabilizes information carried by spatial and temporal spike patterns. Neuron 61, 597-608. doi: 10.1016/j.neuron.2009. 01.008

Keilholz, S. D. (2014). The neural basis of time-varying resting-state functional connectivity. Brain Connect. 4, 769-779. doi: 10.1089/brain. 2014.0250
Keller, D., Erö, C., and Markram, H. (2018). Cell densities in the mouse brain: a systematic review. Front Neuroanat 12:83. doi: 10.3389/fnana.2018.00083

Kelz, M. B., Sun, Y., Chen, J., Cheng Meng, Q., Moore, J. T., Veasey, S. C., et al. (2008). An essential role for orexins in emergence from general anesthesia. Proc. Natl. Acad. Sci. U S A 105, 1309-1314. doi: 10.1073/pnas.070 7146105

Kenny, J. D., Westover, M. B., Ching, S., Brown, E. N., and Solt, K. (2014). Propofol and sevoflurane induce distinct burst suppression patterns in rats. Front. Syst. Neurosci. 8:237. doi: 10.3389/fnsys.2014.00237

Kety, S. S., and Schmidt, C. F. (1948). The effects of altered arterial tensions of carbon dioxide and oxygen on cerebral blood flow and cerebral oxygen consumption of normal young men. J. Clin. Invest. 27, 484-492. doi: $10.1172 /$ jci101995

Kim, S.-G. (2018). Biophysics of BOLD fMRI investigated with animal models. J. Magn. Reson. 292, 82-89. doi: 10.1016/j.jmr.2018.04.006

Kim, J.-H., Jung, A.-H., Jeong, D., Choi, I., Kim, K., Shin, S., et al. (2016). Selectivity of neuromodulatory projections from the basal forebrain and locus ceruleus to primary sensory cortices. J. Neurosci. 36, 5314-5327. doi: 10.1523/JNEUROSCI. 4333-15.2016

Kim, A., Latchoumane, C., Lee, S., Kim, G. B., Cheong, E., Augustine, G. J., et al. (2012). Optogenetically induced sleep spindle rhythms alter sleep architectures in mice. Proc. Natl. Acad. Sci. U S A 109, 20673-20678. doi: 10.1073/pnas. 1217897109

King, P. R., Gundlach, A. L., and Louis, W. J. (1995). Quantitative autoradiographic localization in rat brain of $\alpha 2$-adrenergic and non-adrenergic I-receptor binding sites labelled by [3H]rilmenidine. Brain Res. 675, 264-278. doi: 10.1016/0006-8993(95)00083-3

Kiss, T., Hoffmann, W. E., Scott, L., Kawabe, T. T., Milici, A. J., Nilsen, E. A., et al. (2011). Role of thalamic projection in NMDA receptor-induced disruption of cortical slow oscillation and short-term plasticity. Front. Psychiatry 2:14. doi: 10.3389/fpsyt.2011.00014

Kissinger, S. T., Pak, A., Tang, Y., Masmanidis, S. C., and Chubykin, A. A. (2018). Oscillatory encoding of visual stimulus familiarity. J. Neurosci. 38, 6223-6240. doi: 10.1523/jneurosci.3646-17.2018

Kleckner, I. R., Zhang, J., Touroutoglou, A., Chanes, L., Xia, C., Simmons, W. K., et al. (2017). Evidence for a large-scale brain system supporting allostasis and interoception in humans. Nat. Hum. Behav. 1:0069. doi: 10.1038/s41562-0170069

Klee, R., Mueggler, T., Bruns, A., Kienlin, M., and Künnecke, B. (2017a). "Is sevoflurane a viable alternative anaesthetic for functional MRI in mice?" in Conference Paper: Annual Meeting and Exhibition of the International Society for Magnetic Resonance in Medicine at Volume: 25th (Honolulu, HI).

Klee, R., Mueggler, T., Bruns, A., Wyttenbach, N., Ricci, A., Gasser, R., et al. (2017b). "Etomidate anaesthesia for fmri in mice revisited: subcutaneous administration facilitates experimental procedures," in Conference Paper: Annual Meeting and Exhibition of the International Society for Magnetic Resonance in Medicine at Volume: 25th (Honolulu, HI).

Klimesch, W., Sauseng, P., and Hanslmayr, S. (2007). EEG $\alpha$ oscillations: the inhibition-timing hypothesis. Brain Res. Rev. 53, 63-88. doi: 10.1016/j. brainresrev.2006.06.003

Kozai, T. D., Du, Z., Gugel, Z. V., Smith, M. A., Chase, S. M., Bodily, L. M., et al. (2015). Comprehensive chronic laminar single-unit, multi-unit, and local field potential recording performance with planar single shank electrode arrays. J. Neurosci. Methods 242, 15-40. doi: 10.1016/j.jneumeth.2014. 12.010

Kroeger, D., and Amzica, F. (2007). Hypersensitivity of the anesthesia-induced comatose brain. J. Neurosci. 27, 10597-10607. doi: 10.1523/JNEUROSCI.344007.2007

Ku, S. W., Lee, U., Noh, G. J., Jun, I. G., and Mashour, G. A. (2011). Preferential inhibition of frontal-to-parietal feedback connectivity is a neurophysiologic correlate of general anesthesia in surgical patients. PLoS One 6:e25155. doi: 10.1371/journal.pone.0025155

Kucyi, A., and Davis, K. D. (2017). The neural code for pain: from single-cell electrophysiology to the dynamic pain connectome. Neuroscientist 23, 397-414. doi: $10.1177 / 1073858416667716$

Kundishora, A. J., Gummadavelli, A., Ma, C., Liu, M., Mccafferty, C., Schiff, N. D., et al. (2017). Restoring conscious arousal during focal limbic seizures with 
deep brain stimulation. Cereb. Cortex 27, 1964-1975. doi: 10.1093/cercor/ bhw035

Kuroki, S., Yoshida, T., Tsutsui, H., Iwama, M., Ando, R., Michikawa, T., et al. (2018). Excitatory neuronal hubs configure multisensory integration of slow waves in association cortex. Cell Rep. 22, 2873-2885. doi: 10.1016/j.celrep.2018. 02.056

Lai, H.-Y., Albaugh, D. L., Kao, Y.-C. J., Younce, J. R., and Shih, Y.-Y. I. (2015). Robust deep brain stimulation functional MRI procedures in rats and mice using an MR-compatible tungsten microwire electrode. Magn. Reson. Med. 73, 1246-1251. doi: 10.1002/mrm.25239

Lamme, V. A. F. (2018). Challenges for theories of consciousness: seeing or knowing, the missing ingredient and how to deal with panpsychism. Philos. Trans. R. Soc. Lond. B Biol. Sci. 373:20170344. doi: 10.1098/rstb. 2017.0344

Lamme, V. A. F., Zipser, K., and Spekreijse, H. (1998). Figure-ground activity in primary visual cortex is suppressed by anesthesia. Proc. Natl. Acad. Sci. U S A 95, 3263-3268. doi: 10.1073/pnas.95.6.3263

Land, R., Engler, G., Kral, A., and Engel, A. K. (2012). Auditory evoked bursts in mouse visual cortex during isoflurane anesthesia. PLoS One 7:e49855. doi: 10.1371/journal.pone.0049855

Långsjö, J. W., Alkire, M. T., Kaskinoro, K., Hayama, H., Maksimow, A., Kaisti, K. K., et al. (2012). Returning from oblivion: imaging the neural core of consciousness. J. Neurosci. 32, 4935-4943. doi: 10.1523/JNEUROSCI.496211.2012

Larson-Prior, L. J., Zempel, J. M., Nolan, T. S., Prior, F. W., Snyder, A. Z., and Raichle, M. E. (2009). Cortical network functional connectivity in the descent to sleep. Proc. Natl. Acad. Sci. U S A 106, 4489-4494. doi: 10.1073/pnas. 0900924106

Le Van Quyen, M., Muller, L. E. II., Telenczuk, B., Halgren, E., Cash, S., Hatsopoulos, N. G., et al. (2016). High-frequency oscillations in human and monkey neocortex during the wake-sleep cycle. Proc. Natl. Acad. Sci. U S A 113, 9363-9368. doi: 10.1073/pnas.1523583113

Lecrux, C., and Hamel, E. (2016). Neuronal networks and mediators of cortical neurovascular coupling responses in normal and altered brain states. Philos. Trans. R. Soc. Lond. B Biol. Sci. 371:20150350. doi: 10.1098/rstb.2015.0350

Lecrux, C., Bourourou, M., and Hamel, E. (2019). How reliable is cerebral blood flow to map changes in neuronal activity? Auton. Neurosci. 217, 71-79. doi: 10.1016/j.autneu.2019.01.005

Lee, S.-H., and Dan, Y. (2012). Neuromodulation of brain states. Neuron 76, 209-222. doi: 10.1016/j.neuron.2012.09.012

Lee, J. G., Hudetz, A. G., Smith, J. J., Hillard, C. J., Bosnjak, Z. J., and Kampine, J. P. (1994). The effects of halothane and isoflurane on cerebrocortical microcirculation and autoregulation as assessed by laserDoppler flowmetry. Anesth. Analg. 79, 58-65. doi: 10.1213/00000539199407000-00012

Lee, U., Ku, S., Noh, G., Baek, S., Choi, B., and Mashour, G. A. (2013). Disruption of frontal-parietal communication by ketamine, propofol, and sevoflurane. Anesthesiology 118, 1264-1275. doi: 10.1097/ALN.0b013e31 $829103 \mathrm{f5}$

Lee, V., and Maguire, J. (2014). The impact of tonic $\mathrm{GABA}_{A}$ receptor-mediated inhibition on neuronal excitability varies across brain region and cell type. Front. Neural Circuits 8:3. doi: 10.3389/fncir.2014.00003

Lee, H., Mashour, G. A., Noh, G. J., Kim, S., and Lee, U. (2013). Reconfiguration of network hub structure after propofol-induced unconsciousness. Anesthesiology 119, 1347-1359. doi: 10.1097/aln.0b013e3182a8ec8c

Legrain, V., Iannetti, G. D., Plaghki, L., and Mouraux, A. (2011). The pain matrix reloaded: a salience detection system for the body. Prog. Neurobiol. 93, 111-124. doi: 10.1016/j.pneurobio.2010.10.005

Lehto, L. J., Filip, P., Laakso, H., Sierra, A., Slopsema, J. P., Johnson, M. D., et al. (2018). Tuning neuromodulation effects by orientation selective deep brain stimulation in the rat medial frontal cortex. Front. Neurosci. 12:899. doi: 10.3389/fnins.2018.00899

Lei, H., Grinberg, O., Nwaigwe, C. I., Hou, H. G., Williams, H., Swartz, H. M., et al. (2001). The effects of ketamine-xylazine anesthesia on cerebral blood flow and oxygenation observed using nuclear magnetic resonance perfusion imaging and electron paramagnetic resonance oximetry. Brain Res. 913, 174-179. doi: 10.1016/s0006-8993(01)02786-x
Lein, E. S., Hawrylycz, M. J., Ao, N., Ayres, M., Bensinger, A., Bernard, A., et al. (2007). Genome-wide atlas of gene expression in the adult mouse brain. Nature 445, 168-176. doi: 10.1038/nature05453

Leong, A. T. L., Chan, R. W., Gao, P. P., Chan, Y.-S., Tsia, K. K., Yung, W.-H., et al. (2016). Long-range projections coordinate distributed brain-wide neural activity with a specific spatiotemporal profile. Proc. Natl. Acad. Sci. U S A 113, E8306-E8315. doi: 10.1073/pnas.1616361113

Leoni, R. F., Paiva, F. F., Henning, E. C., Nascimento, G. C., Tannus, A., de Araujo, D. B., et al. (2011). Magnetic resonance imaging quantification of regional cerebral blood flow and cerebrovascular reactivity to carbon dioxide in normotensive and hypertensive rats. NeuroImage 58, 75-81. doi: 10.1016/j. neuroimage.2011.06.030

Leung, L. S., Luo, T., Ma, J., and Herrick, I. (2014). Brain areas that influence general anesthesia. Prog. Neurobiol. 122, 24-44. doi: 10.1016/j.pneurobio.2014. 08.001

Lewis, L. D., and Akeju, O. (2017). Hierarchy in disruption of largescale networks across altered arousal states. Br. J. Anaesth. 119, 566-568. doi: 10.1093/bja/aex297

Lewis, L. D., Ching, S., Weiner, V. S., Peterfreund, R. A., Eskandar, E. N., Cash, S. S., et al. (2013). Local cortical dynamics of burst suppression in the anaesthetized brain. Brain 136, 2727-2737. doi: 10.1093/brain/awt174

Lewis, L. D., Voigts, J., Flores, F. J., Schmitt, L. I., Wilson, M. A., Halassa, M. M., et al. (2015). Thalamic reticular nucleus induces fast and local modulation of arousal state. Elife 4:e08760. doi: 10.7554/eLife.08760

Lewis, L. D., Weiner, V. S., Mukamel, E. A., Donoghue, J. A., Eskandar, E. N., Madsen, J. R., et al. (2012). Rapid fragmentation of neuronal networks at the onset of propofol-induced unconsciousness. Proc. Natl. Acad. Sci. U S A 109, E3377-E3386. doi: 10.1073/pnas.1210907109

Li, Q., Li, G., Wu, D., Lu, H., Hou, Z., Ross, C. A., et al. (2017). Resting-state functional MRI reveals altered brain connectivity and its correlation with motor dysfunction in a mouse model of Huntington's disease. Sci. Rep. 7:16742. doi: 10.1038/s41598-017-17026-5

Li, B. H., Lohmann, J. S., Schuler, H. G., and Cronin, A. J. (2003). Preservation of the cortical somatosensory-evoked potential during dexmedetomidine infusion in rats. Anesth. Analg. 96, 1155-1160. doi: 10.1213/01.ane.0000053239. 62623.32

Lichtner, G., Auksztulewicz, R., Kirilina, E., Velten, H., Mavrodis, D., Scheel, M., et al. (2018). Effects of propofol anesthesia on the processing of noxious stimuli in the spinal cord and the brain. NeuroImage 172, 642-653. doi: 10.1016/j. neuroimage.2018.02.003

Liska, A., Galbusera, A., Schwarz, A. J., and Gozzi, A. (2015). Functional connectivity hubs of the mouse brain. NeuroImage 115, 281-291. doi: 10.1016/j. neuroimage.2015.04.033

Liu, X., Lauer, K. K., Ward, B. D., Li, S. J., and Hudetz, A. G. (2013a). Differential effects of deep sedation with propofol on the specific and nonspecific thalamocortical systems: a functional magnetic resonance imaging study. Anesthesiology 118, 59-69. doi: 10.1097/ALN.0b013e318277a801

Liu, X., Pillay, S., Li, R., Vizuete, J. A., Pechman, K. R., Schmainda, K. M., et al. (2013b). Multiphasic modification of intrinsic functional connectivity of the rat brain during increasing levels of propofol. NeuroImage 83, 581-592. doi: 10.1016/j.neuroimage.2013.07.003

Liu, X., Zhu, X. H., Zhang, Y., and Chen, W. (2013c). The change of functional connectivity specificity in rats under various anesthesia levels and its neural origin. Brain Topogr. 26, 363-377. doi: 10.1007/s10548-012-0267-5

Liu, X., Zhu, X. H., Zhang, Y., and Chen, W. (2011). Neural origin of spontaneous hemodynamic fluctuations in rats under burst-suppression anesthesia condition. Cereb. Cortex 21, 374-384. doi: 10.1093/cercor/ bhq105

Llinás, R., and Ribary, U. (1993). Coherent 40-Hz oscillation characterizes dream state in humans. Proc. Natl. Acad. Sci. U S A 90, 2078-2081. doi: 10.1073/pnas. 90.5.2078

Longmuir, I. S., and Pashko, L. (1976). The induction of cytochrome P-450 by hypoxia. Adv. Exp. Med. Biol. 75, 171-175. doi: 10.1007/978-1-46843273-2_22

Low, L. A., Bauer, L. C., and Klaunberg, B. A. (2016a). Comparing the effects of isoflurane and $\alpha$ chloralose upon mouse physiology. PLoS One 11:e0154936. doi: 10.1371/journal.pone.0154936 
Low, L. A., Bauer, L. C., Pitcher, M. H., and Bushnell, M. C. (2016b). Restraint training for awake functional brain scanning of rodents can cause long-lasting changes in pain and stress responses. Pain 157, 1761-1772. doi: 10.1097/j.pain. 0000000000000579

Lu, H., Zou, Q., Gu, H., Raichle, M. E., Stein, E. A., and Yang, Y. (2012). Rat brains also have a default mode network. Proc. Natl. Acad. Sci. U S A 109, 3979-3984. doi: 10.1073/pnas.1200506109

Luczak, A., Barthó, P., Marguet, S. L., Buzsáki, G., and Harris, K. D. (2007). Sequential structure of neocortical spontaneous activity in vivo. Proc. Natl. Acad. Sci. U S A 104, 347-352. doi: 10.1073/pnas.0605643104

Luczak, A., McNaughton, B. L., and Harris, K. D. (2015). Packetbased communication in the cortex. Nat. Rev. Neurosci. 16, 745-755. doi: 10.1038/nrn4026

Lurie, J. D., Kessler, D., Bassett, D., Betzel, R., Breakspear, M., Keilholz, S., et al. (2018). On the nature of resting fMRI and time-varying functional connectivity. PsyArXiv [Preprint].

Lüthi, A. (2014). Sleep spindles: where they come from, what they do. Neuroscientist 20, 243-256. doi: 10.1177/1073858413500854

Ma, S., Allocca, G., Ong-Palsson, E. K., Singleton, C. E., Hawkes, D., McDougall, S. J., et al. (2017). Nucleus incertus promotes cortical desynchronization and behavioral arousal. Brain Struct. Funct. 222, 515-537. doi: 10.1007/s00429-016-1230-0

Ma, S., Hangya, B., Leonard, C. S., Wisden, W., and Gundlach, A. L. (2018). Dual-transmitter systems regulating arousal, attention, learning and memory. Neurosci. Biobehav. Rev. 85, 21-33. doi: 10.1016/j.neubiorev.2017.07.009

Madularu, D., Mathieu, A. P., Kumaragamage, C., Reynolds, L. M., Near, J., Flores, C., et al. (2017). A non-invasive restraining system for awake mouse imaging. J. Neurosci. Methods 287, 53-57. doi: 10.1016/j.jneumeth.2017.06.008

Mahdavi, A., Qin, Y., Aubry, A.-S., Cornec, D., Kulikova, S., and Pinault, D. (2019). Sleep-related thalamocortical spindles and delta oscillations are reduced during a ketamine-induced psychosis-relevant transition state. bioRxiv [Preprint]. doi: 10.1101/833459

Majeed, W., Magnuson, M., Hasenkamp, W., Schwarb, H., Schumacher, E. H., Barsalou, L., et al. (2011). Spatiotemporal dynamics of low frequency BOLD fluctuations in rats and humans. NeuroImage 54, 1140-1150. doi: 10.1016/j. neuroimage.2010.08.030

Mandino, F., Cerri, D. H., Garin, C. M., Straathof, M., van Tilborg, G. A. F., Chakravarty, M. M., et al. (2020). Animal functional magnetic resonance imaging: trends and path toward standardization. Front. Neuroinform. 13:78. doi: 10.3389/fninf.2019.00078

Marlinski, V., Sirota, M. G., and Beloozerova, I. N. (2012). Differential gating of thalamocortical signals by reticular nucleus of thalamus during locomotion. J. Neurosci. 32, 15823-15836. doi: 10.1523/JNEUROSCI.078212.2012

Martins, I., and Tavares, I. (2017). Reticular formation and pain: the past and the future. Front. Neuroanat. 11:51. doi: 10.3389/fnana.2017.00051

Masamoto, K., and Kanno, I. (2012). Anesthesia and the quantitative evaluation of neurovascular coupling. J. Cereb. Blood Flow Metab. 32, 1233-1247. doi: $10.1038 /$ jcbfm.2012.50

Mashour, G. A. (2014). Top-down mechanisms of anesthetic-induced unconsciousness. Front. Syst. Neurosci. 8:115. doi: 10.3389/fnsys.2014. 00115

Mashour, G. A., and Alkire, M. T. (2013). Evolution of consciousness: phylogeny, ontogeny, and emergence from general anesthesia. Proc. Natl. Acad. Sci. U S A 110, 10357-10364. doi: 10.1073/pnas.1301188110

Mashour, G. A., and Hudetz, A. G. (2017). Bottom-up and top-down mechanisms of general anesthetics modulate different dimensions of consciousness. Front. Neural Circuits 11:44. doi: 10.3389/fncir.2017.00044

Mashour, G. A., and Hudetz, A. G. (2018). Neural correlates of unconsciousness in large-scale brain networks. Trends Neurosci. 41, 150-160. doi: 10.1016/j.tins. 2018.01.003

Masri, R., Trageser, J. C., Bezdudnaya, T., Li, Y., and Keller, A. (2006). Cholinergic regulation of the posterior medial thalamic nucleus. J. Neurophysiol. 96, 2265-2273. doi: 10.1152/jn.00476.2006

Masri, R., Bezdudnaya, T., Trageser, J. C., and Keller, A. (2008). Encoding of stimulus frequency and sensor motion in the posterior medial thalamic nucleus. J. Neurophysiol. 100, 681-689. doi: 10.1152/jn.01322.2007
Massimini, M., Ferrarelli, F., Esser, S. K., Riedner, B. A., Huber, R., Murphy, M., et al. (2007). Triggering sleep slow waves by transcranial magnetic stimulation. Proc. Natl. Acad. Sci. U S A 104, 8496-8501. doi: 10.1073/pnas.0702495104

Massimini, M., Ferrarelli, F., Huber, R., Esser, S. K., Singh, H., and Tononi, G. (2005). Breakdown of cortical effective connectivity during sleep. Science 309, 2228-2232. doi: 10.1126/science.1117256

Massimini, M., Huber, R., Ferrarelli, F., Hill, S., and Tononi, G. (2004). The sleep slow oscillation as a traveling wave. J. Neurosci. 24, 6862-6870. doi: 10.1523/JNEUROSCI.1318-04.2004

Mateo, C., Knutsen, P. M., Tsai, P. S., Shih, A. Y., and Kleinfeld, D. (2017). Entrainment of arteriole vasomotor fluctuations by neural activity is a basis of blood-oxygenation-level-dependent "resting-state" connectivity. Neuron 96, 936.e3-948.e3. doi: 10.1016/j.neuron.2017.10.012

Matsui, T., Murakami, T., and Ohki, K. (2018). Neuronal origin of the temporal dynamics of spontaneous BOLD activity correlation. Cereb. Cortex 29, 1496-1508. doi: 10.1093/cercor/bhy045

Matsuura, T., and Kanno, I. (2002). Effect of nitric oxide synthase inhibitor on the local cerebral blood flow evoked by rat somatosensory stimulation under hyperoxia. Comp. Biochem. Physiol. A Mol. Integr. Physiol. 131, 267-274. doi: 10.1016/s1095-6433(01)00450-0

Matsuura, T., Kashikura, K., and Kanno, I. (2001). Hemodynamics of local cerebral blood flow induced by somatosensory stimulation under normoxia and hyperoxia in rats. Comp. Biochem. Physiol. A Mol. Integr. Physiol. 129, 363-372. doi: 10.1016/s1095-6433(00)00354-8

Mattia, M., and Sanchez-Vives, M. V. (2012). Exploring the spectrum of dynamical regimes and timescales in spontaneous cortical activity. Cogn. Neurodyn. 6 , 239-250. doi: 10.1007/s11571-011-9179-4

Maxwell, C. R., Ehrlichman, R. S., Liang, Y., Trief, D., Kanes, S. J., Karp, J., et al. (2006). Ketamine produces lasting disruptions in encoding of sensory stimuli. J. Pharmacol. Exp. Ther. 316, 315-324. doi: 10.1124/jpet.105.091199

McCallum, J. B., Boban, N., Hogan, Q., Schmeling, W. T., Kampine, J. P., and Bosnjak, Z. J. (1998). The mechanism of $\alpha 2$-adrenergic inhibition of sympathetic ganglionic transmission. Anesth. Analg. 87, 503-510. doi: 10.1097/00000539-199809000-00001

McCarthy, M. M., Brown, E. N., and Kopell, N. (2008). Potential network mechanisms mediating electroencephalographic $\beta$ rhythm changes during propofol-induced paradoxical excitation. J. Neurosci. 28, 13488-13504. doi: 10.1523/JNEUROSCI.3536-08.2008

McCormick, D. A. (1992). Neurotransmitter actions in the thalamus and cerebral cortex and their role in neuromodulation of thalamocortical activity. Prog. Neurobiol. 39, 337-388. doi: 10.1016/0301-0082(92)90012-4

McCormick, D. A., and Bal, T. (1997). Sleep and arousal: thalamocortical mechanisms. Ann. Rev. Neurosci. 20, 185-215. doi: 10.1146/annurev.neuro.20. 1.185

McCormick, D. A., and Pape, H. C. (1990). Properties of a hyperpolarizationactivated cation current and its role in rhythmic oscillation in thalamocortical neurones. J. Physiol. 431, 291-318. doi: 10.1113/jphysiol.1990. sp018331

McGinley, M. J., David, S. V., and McCormick, D. A. (2015a). Cortical membrane potential signature of optimal states for sensory signal detection. Neuron 87 , 179-192. doi: 10.1016/j.neuron.2015.05.038

McGinley, M. J., Vinck, M., Reimer, J., Batista-Brito, R., Zagha, E., Cadwell, C. R., et al. (2015b). Waking state: rapid variations modulate neural and behavioral responses. Neuron 87, 1143-1161. doi: 10.1016/j.neuron.2015.09.012

McIlhone, A. E., Beausoleil, N. J., Kells, N. J., Johnson, C. B., and Mellor, D. J. (2018). Effects of halothane on the electroencephalogram of the chicken. Vet. Med. Sci. 4, 98-105. doi: 10.1002/vms3.91

McIntyre, C. C., and Anderson, R. W. (2016). Deep brain stimulation mechanisms: the control of network activity via neurochemistry modulation. J. Neurochem. 139, 338-345. doi: 10.1111/jnc.13649

McKinstry-Wu, A., and Kelz, M. B. (2019). Neural Mechanisms of Anaesthetics. Oxford Textbook of Neuroscience and Anaesthesiology. New York, NY: Oxford University Press.

McVea, D. A., Murphy, T. H., and Mohajerani, M. H. (2016). Large scale cortical functional networks associated with slow-wave and spindle-burstrelated spontaneous activity. Front. Neural Circuits 10:103. doi: 10.3389/fncir. 2016.00103 
Mechling, A. E., Hubner, N. S., Lee, H. L., Hennig, J., von Elverfeldt, D., and Harsan, L. A. (2014). Fine-grained mapping of mouse brain functional connectivity with resting-state fMRI. NeuroImage 96, 203-215. doi: 10.1016/j. neuroimage.2014.03.078

Melzack, R. (1989). Labat lecture. Phantom limbs. Reg. Anesth. 14, 208-211.

Melzack, R. (2005). Evolution of the neuromatrix theory of pain. The Prithvi Raj Lecture: presented at the third World Congress of World Institute of Pain, Barcelona 2004. Pain Pract. 5, 85-94. doi: 10.1111/j.1533-2500.2005.05203.x

Meuth, S. G., Budde, T., Kanyshkova, T., Broicher, T., Munsch, T., and Pape, H. C. (2003). Contribution of TWIK-related acid-sensitive $\mathrm{K}^{+}$channel 1 (TASK1) and TASK3 channels to the control of activity modes in thalamocortical neurons. J. Neurosci. 23, 6460-6469. doi: 10.1523/JNEUROSCI.23-16-06 460.2003

Meuth, S. G., Kanyshkova, T., Meuth, P., Landgraf, P., Munsch, T., Ludwig, A., et al. (2006). Membrane resting potential of thalamocortical relay neurons is shaped by the interaction among TASK3 and HCN2 channels. J. Neurophysiol. 96, 1517-1529. doi: 10.1152/jn.01212.2005

Meyer, H. S., Egger, R., Guest, J. M., Foerster, R., Reissl, S., and Oberlaender, M. (2013). Cellular organization of cortical barrel columns is whisker-specific. Proc. Natl. Acad. Sci. U S A 110, 19113-19118. doi: 10.1073/pnas. 1312691110

Michalareas, G., Vezoli, J., van Pelt, S., Schoffelen, J. M., Kennedy, H., and Fries, P. (2016). Alpha-beta and gamma rhythms subserve feedback and feedforward influences among human visual cortical areas. Neuron 89, 384-397. doi: 10. 1016/j.neuron.2015.12.018

Michelson, N. J., and Kozai, T. D. Y. (2018). Isoflurane and ketamine differentially influence spontaneous and evoked laminar electrophysiology in mouse V1. J. Neurophysiol. 120, 2232-2245. doi: 10.1152/jn.00299.2018

Milenkovic, N., Zhao, W.-J., Walcher, J., Albert, T., Siemens, J., Lewin, G. R., et al. (2014). A somatosensory circuit for cooling perception in mice. Nat. Neurosci. 17, 1560-1566. doi: 10.1038/nn.3828

Mizobe, T. (2019). The halothane hepatitis that was not. Br. J. Anaesth. 124, e2-e3. doi: 10.1016/j.bja.2019.09.018

Mo, C., and Sherman, S. M. (2019). A sensorimotor pathway via higherorder thalamus. J. Neurosci. 39, 692-704. doi: 10.1523/JNEUROSCI.146718.2018

Moghaddam, B., Adams, B., Verma, A., and Daly, D. (1997). Activation of glutamatergic neurotransmission by ketamine: a novel step in the pathway from NMDA receptor blockade to dopaminergic and cognitive disruptions associated with the prefrontal cortex. J. Neurosci. 17, 2921-2927. doi: 10.1523/JNEUROSCI.17-08-02921.1997

Mohajerani, M. H., Chan, A. W., Mohsenvand, M., LeDue, J., Liu, R., McVea, D. A., et al. (2013). Spontaneous cortical activity alternates between motifs defined by regional axonal projections. Nat. Neurosci. 16, 1426-1435. doi: $10.1038 / \mathrm{nn} .3499$

Mölle, M., Marshall, L., Gais, S., and Born, J. (2002). Grouping of spindle activity during slow oscillations in human non-rapid eye movement sleep. J. Neurosci. 22, 10941-10947. doi: 10.1523/JNEUROSCI.22-24-10941.2002

Montemurro, M. A., Rasch, M. J., Murayama, Y., Logothetis, N. K., and Panzeri, S. (2008). Phase-of-firing coding of natural visual stimuli in primary visual cortex. Curr. Biol. 18, 375-380. doi: 10.1016/j.cub.2008.02.023

Monti, M. M., Lutkenhoff, E. S., Rubinov, M., Boveroux, P., Vanhaudenhuyse, A., Gosseries, O., et al. (2013). Dynamic change of global and local information processing in propofol-induced loss and recovery of consciousness. PLoS Comput. Biol. 9:e1003271. doi: 10.1371/journal.pcbi.1003271

Montijn, J. S., Meijer, G. T., Lansink, C. S., and Pennartz, C. M. A. (2016). Population-level neural codes are robust to single-neuron variability from a multidimensional coding perspective. Cell Rep. 16, 2486-2498. doi: 10.1016/j. celrep.2016.07.065

Moon, J. Y., Lee, U., Blain-Moraes, S., and Mashour, G. A. (2015). General relationship of global topology, local dynamics, and directionality in largescale brain networks. PLoS Comput. Biol. 11:e1004225. doi: 10.1371/journal. pcbi. 1004225

Mouraux, A., Diukova, A., Lee, M. C., Wise, R. G., and Iannetti, G. D. (2011). A multisensory investigation of the functional significance of the "pain matrix". NeuroImage 54, 2237-2249. doi: 10.1016/j.neuroimage.2010.09.084

Mouraux, A., and Iannetti, G. D. (2018). The search for pain biomarkers in the human brain. Brain 141, 3290-3307. doi: 10.1093/brain/awy281
Mueggler, T., Baumann, D., Rausch, M., Staufenbiel, M., and Rudin, M. (2003). Age-dependent impairment of somatosensory response in the amyloid precursor protein 23 transgenic mouse model of Alzheimer's disease. J. Neurosci. 23, 8231-8236. doi: 10.1523/JNEUROSCI.23-23-08231.2003

Mukamel, E. A., Pirondini, E., Babadi, B., Wong, K. F., Pierce, E. T., Harrell, P. G., et al. (2014). A transition in brain state during propofol-induced unconsciousness. J. Neurosci. 34, 839-845. doi: 10.1523/JNEUROSCI.581312.2014

Muller, L., Chavane, F., Reynolds, J., and Sejnowski, T. J. (2018). Cortical travelling waves: mechanisms and computational principles. Nat. Rev. Neurosci. 19 , 255-268. doi: 10.1038/nrn.2018.20

Munting, L. P., Derieppe, M. P. P., Suidgeest, E., Denis de Senneville, B., Wells, J., and van der Weerd, L. (2019). Influence of different isoflurane anesthesia protocols on murine cerebral hemodynamics measured with pseudo-continuous arterial spin labeling. NMR Biomed. 32:e4105. doi: 10.1002/ nbm.4105

Muthukumaraswamy, S. D., Shaw, A. D., Jackson, L. E., Hall, J., Moran, R., and Saxena, N. (2015). Evidence that subanesthetic doses of ketamine cause sustained disruptions of NMDA and AMPA-mediated frontoparietal connectivity in humans. J. Neurosci. 35, 11694-11706. doi: 10.1523/JNEUROSCI.0903-15.2015

Murphy, M. C., Chan, K. C., Kim, S.-G., and Vazquez, A. L. (2017). Macroscale variation in resting-state neuronal activity and connectivity assessed by simultaneous calcium imaging, hemodynamic imaging and electrophysiology. NeuroImage 169, 352-362. doi: 10.1016/j.neuroimage.2017. 12.070

Murphy, C., Krause, B., and Banks, M. (2019). Selective effects of isoflurane on cortico-cortical feedback afferent responses in murine non-primary neocortex. Br. J. Anaesth. 123, 488-496. doi: 10.1016/j.bja.2019.06.018

Murrell, J. C., Waters, D., and Johnson, C. B. (2008). Comparative effects of halothane, isoflurane, sevoflurane and desflurane on the electroencephalogram of the rat. Lab. Anim. 42, 161-170. doi: 10.1258/la.2007.06019e

Nasrallah, F. A., Lew, S. K., Low, A. S., and Chuang, K. H. (2014a). Neural correlate of resting-state functional connectivity under $\alpha 2$ adrenergic receptor agonist, medetomidine. NeuroImage 84, 27-34. doi: 10.1016/j.neuroimage.2013. 08.004

Nasrallah, F. A., Tay, H. C., and Chuang, K. H. (2014b). Detection of functional connectivity in the resting mouse brain. NeuroImage 86, 417-424. doi: 10.1016/j.neuroimage.2013.10.025

Nasrallah, F. A., Tan, J., and Chuang, K. H. (2012). Pharmacological modulation of functional connectivity: $\alpha 2$-adrenergic receptor agonist alters synchrony but not neural activation. NeuroImage 60, 436-446. doi: 10.1016/j.neuroimage. 2011.12.026

Nelson, L. E., Lu, J., Guo, T., Saper, C. B., Franks, N. P., and Maze, M. (2003). The $\alpha 2$-adrenoceptor agonist dexmedetomidine converges on an endogenous sleeppromoting pathway to exert its sedative effects. Anesthesiology 98, 428-436. doi: 10.1097/00000542-200302000-00024

Nelson, C. L., Sarter, M., and Bruno, J. P. (2005). Prefrontal cortical modulation of acetylcholine release in posterior parietal cortex. Neuroscience 132, 347-359. doi: 10.1016/j.neuroscience.2004.12.007

Neske, G. T. (2015). The slow oscillation in cortical and thalamic networks: mechanisms and functions. Front. Neural Circuits 9:88. doi: 10.3389/fncir.2015. 00088

Ní Mhuircheartaigh, R., Warnaby, C., Rogers, R., Jbabdi, S., and Tracey, I. (2013). Slow-wave activity saturation and thalamocortical isolation during propofol anesthesia in humans. Sci. Transl. Med. 5:208ra148. doi: 10.1126/scitranslmed. 3006007

Niedermeyer, E., Sherman, D. L., Geocadin, R. J., Hansen, H. C., and Hanley, D. F. (1999). The burst-suppression electroencephalogram. Clin. Electroencephalogr. 30, 99-105. doi: 10.1177/155005949903000305

Niendorf, T., Pohlmann, A., Reimann, H. M., Waiczies, H., Peper, E., Huelnhagen, T., et al. (2015). Advancing cardiovascular, neurovascular and renal magnetic resonance imaging in small rodents using cryogenic radiofrequency coil technology. Front. Pharmacol. 6:255. doi: 10.3389/fphar. 2015.00255

Nir, Y., Staba, R. J., Andrillon, T., Vyazovskiy, V. V., Cirelli, C., Fried, I., et al. (2011). Regional slow waves and spindles in human sleep. Neuron 70, 153-169. doi: 10.1016/j.neuron.2011.02.043 
Niranjan, A., Christie, I. N., Solomon, S. G., Wells, J. A., and Lythgoe, M. F. (2016). fMRI mapping of the visual system in the mouse brain with interleaved snapshot GE-EPI. NeuroImage 139, 337-345. doi: 10.1016/j.neuroimage.2016. 06.015

Noreika, V., Jylhänkangas, L., Móró, L., Valli, K., Kaskinoro, K., Aantaa, R., et al. (2011). Consciousness lost and found: subjective experiences in an unresponsive state. Brain Cogn. 77, 327-334. doi: 10.1016/j.bandc.2011.09.002

Nuñez, A., Amzica, F., and Steriade, M. (1992). Voltage-dependent fast (20-40 Hz) oscillations in long-axoned neocortical neurons. Neuroscience 51, 7-10. doi: 10.1016/0306-4522(92)90464-d

Ogawa, S., Lee, T. M., Kay, A. R., and Tank, D. W. (1990). Brain magnetic resonance imaging with contrast dependent on blood oxygenation. Proc. Natl. Acad. Sci. U S A 87, 9868-9872. doi: 10.1073/pnas.87.24.9868

Ojemann, G. A., Ramsey, N. F., and Ojemann, J. (2013). Relation between functional magnetic resonance imaging (fMRI) and single neuron, local field potential (LFP) and electrocorticography (ECoG) activity in human cortex. Front. Hum. Neurosci. 7:34. doi: 10.3389/fnhum.2013.00034

Ojemann, G. A., Schoenfield-McNeill, J., and Corina, D. (2002). Anatomic subdivisions in human temporal cortical neuronal activity related to recent verbal memory. Nat. Neurosci. 5, 64-71. doi: 10.1038/nn785

Olcese, U., Oude Lohuis, M. N., and Pennartz, C. M. A. (2018). Sensory processing across conscious and nonconscious brain states: from single neurons to distributed networks for inferential representation. Front. Syst. Neurosci. 12:49. doi: 10.3389/fnsys.2018.00049

Olufsen, M., Whittington, M. A., Camperi, M., and Kopell, N. (2003). New functions for the gamma rhythm: population tuning and preprocessing for the beta rhythm. J. Comput. Neurosci. 14, 33-54. doi: 10.1023/a:1021124 317706

Oye, I., Paulsen, O., and Maurset, A. (1992). Effects of ketamine on sensory perception: evidence for a role of N-methyl-D-aspartate receptors. J. Pharmacol. Exp. Ther. 260, 1209-1213.

Paasonen, J., Laakso, H., Pirttimäki, T., Stenroos, P., Salo, R. A., Zhurakovskaya, E., et al. (2020). Multi-band SWIFT enables quiet and artefact-free EEG-fMRI and awake fMRI studies in rat. NeuroImage 206:116338. doi: 10.1016/j.neuroimage. 2019.116338

Paasonen, J., Stenroos, P., Salo, R. A., Kiviniemi, V., and Grohn, O. (2018). Functional connectivity under six anesthesia protocols and the awake condition in rat brain. NeuroImage 172, 9-20. doi: 10.1016/j.neuroimage.2018. 01.014

Pachitariu, M., Lyamzin, D. R., Sahani, M., and Lesica, N. A. (2015). Statedependent population coding in primary auditory cortex. J. Neurosci. 35, 2058-2073. doi: 10.1523/JNEUROSCI.3318-14.2015

Pack, C. C., Berezovskii, V. K., and Born, R. T. (2001). Dynamic properties of neurons in cortical area MT in alert and anaesthetized macaque monkeys. Nature 414, 905-908. doi: 10.1038/414905a

Pais-Roldán, P., Biswal, B., Scheffler, K., and Yu, X. (2018). Identifying respirationrelated aliasing artifacts in the rodent resting-state fMRI. Front. Neurosci. 12:788. doi: 10.3389/fnins.2018.00788

Pal, D., Dean, J. G., Liu, T., Li, D., Watson, C. J., Hudetz, A. G., et al. (2018). Differential role of prefrontal and parietal cortices in controlling level of consciousness. Curr. Biol. 28, 2145.e5-2152.e5. doi: 10.1016/j.cub.2018. 05.025

Pal, D., Hambrecht-Wiedbusch, V. S., Silverstein, B. H., and Mashour, G. A. (2015). Electroencephalographic coherence and cortical acetylcholine during ketamine-induced unconsciousness. Br. J. Anaesth. 114, 979-989. doi: 10.1093/bja/aev095

Palanca, B. J., Mitra, A., Larson-Prior, L., Snyder, A. Z., Avidan, M. S., and Raichle, M. E. (2015). Resting-state functional magnetic resonance imaging correlates of sevoflurane-induced unconsciousness. Anesthesiology 123, 346-356. doi: 10.1097/aln.0000000000000731

Palva, S., Linkenkaer-Hansen, K., Näätänen, R., and Palva, J. M. (2005). Early neural correlates of conscious somatosensory perception. J. Neurosci. 25, 5248-5258. doi: 10.1523/jneurosci.0141-05.2005

Palva, S., and Palva, J. M. (2007). New vistas for $\alpha$-frequency band oscillations. Trends Neurosci. 30, 150-158. doi: 10.1016/j.tins.2007.02.001

Pan, W. J., Billings, J. C., Grooms, J. K., Shakil, S., and Keilholz, S. D. (2015). Considerations for resting state functional MRI and functional connectivity studies in rodents. Front. Neurosci. 9:269. doi: 10.3389/fnins.2015. 00269

Pan, W. J., Billings, J., Nezafati, M., Abbas, A., and Keilholz, S. (2018). Resting state fMRI in rodents. Curr. Protoc. Neurosci. 83:e45. doi: 10.1002/cpns.45

Paquette, T., Jeffrey-Gauthier, R., Leblond, H., and Pich, E. M. (2018). Functional neuroimaging of nociceptive and pain-related activity in the spinal cord and brain: insights from neurovascular coupling studies. Anat. Rec. 301, 1585-1595. doi: 10.1002/ar.23854

Paquette, T., Tokunaga, R., Touj, S., Leblond, H., and Piché, M. (2019). Regulation of cortical blood flow responses by the nucleus basalis of Meynert during nociceptive processing. Neurosci. Res. 149, 22-28. doi: 10.1016/j.neures.2019. 01.008

Pavone, K. J., Su, L., Gao, L., Eromo, E., Vazquez, R., Rhee, J., et al. (2017). Lack of responsiveness during the onset and offset of sevoflurane anesthesia is associated with decreased awake- $\alpha$ oscillation power. Front. Syst. Neurosci. 11:38. doi: 10.3389/fnsys.2017.00038

Pawela, C. P., Biswal, B. B., Cho, Y. R., Kao, D. S., Li, R., Jones, S. R., et al. (2008). Resting-state functional connectivity of the rat brain. Magn. Reson. Med. 59, 1021-1029. doi: 10.1002/mrm.21524

Pennartz, C. M. A., Dora, S., Muckli, L., and Lorteije, J. A. M. (2019). Towards a Unified View on Pathways and Functions of Neural Recurrent Processing. Trends Neurosci. 42. doi: 10.1016/j.tins.2019.07.005

Petersen, C. C. H., Hahn, T. T. G., Mehta, M., Grinvald, A., and Sakmann, B. (2003). Interaction of sensory responses with spontaneous depolarization in layer 2/3 barrel cortex. Proc. Natl. Acad. Sci. U S A 100, 13638-13643. doi: $10.1073 /$ pnas.2235811100

Petersen, S. E., and Sporns, O. (2015). Brain networks and cognitive architectures. Neuron 88, 207-219. doi: 10.1016/j.neuron.2015.09.027

Petrinovic, M. M., Hankov, G., Schroeter, A., Bruns, A., Rudin, M., Von Kienlin, M., et al. (2016). A novel anesthesia regime enables neurofunctional studies and imaging genetics across mouse strains. Sci. Rep. 6:24523. doi: 10.1038/srep24523

Peyrache, A., Battaglia, F. P., and Destexhe, A. (2011). Inhibition recruitment in prefrontal cortex during sleep spindles and gating of hippocampal inputs. Proc. Natl. Acad. Sci. U S A 108, 17207-17212. doi: 10.1073/pnas.11036 12108

Pfaff, D. (2005). Brain Arousal and Information Theory: Neural and Genetic Mechanisms. Cambridge, MA: Harvard University Press. page 71-72, 123.

Phillips, W. A., Bachmann, T., and Storm, J. F. (2018). Apical function in neocortical pyramidal cells: a common pathway by which general anesthetics can affect mental state. Front. Neural Circuits 12:50. doi: 10.3389/fncir.2018. 00050

Picard, N., Takesian, A. E., Fagiolini, M., and Hensch, T. K. (2019). NMDA 2A receptors in parvalbumin cells mediate sex-specific rapid ketamine response on cortical activity. Mol. Psychiatry 24, 828-838. doi: 10.1038/s41380-0180341-9

Picchioni, D., Reith, R. M., Nadel, J. L., and Smith, C. B. (2014). Sleep, plasticity and the pathophysiology of neurodevelopmental disorders: the potential roles of protein synthesis and other cellular processes. Brain Sci. 4, 150-201. doi: 10.3390/brainsci4010150

Polack, P.-O., Friedman, J., and Golshani, P. (2013). Cellular mechanisms of brain state-dependent gain modulation in visual cortex. Nat. Neurosci. 16, 1331-1339. doi: 10.1038/nn.3464

Porro, C. A., Cavazzuti, M., Giuliani, D., Vellani, V., Lui, F., and Baraldi, P. (2004). Effects of ketamine anesthesia on central nociceptive processing in the rat: a 2-deoxyglucose study. Neuroscience 125, 485-494. doi: 10.1016/j.neuroscience. 2004.01.039

Poulet, J. F. A., and Crochet, S. (2019). The cortical states of wakefulness. Front. Syst. Neurosci. 12:64. doi: 10.3389/fnsys.2018.00064

Prerau, M. J., Brown, R. E., Bianchi, M. T., Ellenbogen, J. M., and Purdon, P. L. (2017). Sleep neurophysiological dynamics through the lens of multitaper spectral analysis. Physiology 32, 60-92. doi: 10.1152/physiol. 00062.2015

Prerau, M. J., Hartnack, K. E., Obregon-Henao, G., Sampson, A., Merlino, M., Gannon, K., et al. (2014). Tracking the sleep onset process: an empirical model of behavioral and physiological dynamics. PLoS Comput. Biol. 10:e1003866. doi: 10.1371/journal.pcbi.1003866 
Preti, M. G., Bolton, T. A., and Van De Ville, D. (2017). The dynamic functional connectome: state-of-the-art and perspectives. NeuroImage 160, 41-54. doi: 10.1016/j.neuroimage.2016.12.061

Price, D. D. (2000). Psychological and neural mechanisms of the affective dimension of pain. Science 288, 1769-1772. doi: 10.1126/science.288. 5472.1769

Pries, A. R., Secomb, T. W., and Gaehtgens, P. (1995). Design principles of vascular beds. Circ. Res. 77, 1017-1023. doi: 10.1161/01.res.77.5.1017

Proekt, A., and Hudson, A. E. (2018). A stochastic basis for neural inertia in emergence from general anaesthesia. Br. J. Anaesth. 121, 86-94. doi: 10.1016/j. bja.2018.02.035

Proekt, A., and Kelz, M. (2018). Schrödinger's cat: anaesthetised and not! Br. J. Anaesth. 120, 424-428. doi: 10.1016/j.bja.2017.11.068

Purdon, P. L., Pierce, E. T., Bonmassar, G., Walsh, J., Harrell, P. G., Kwo, J., et al. (2009). Simultaneous electroencephalography and functional magnetic resonance imaging of general anesthesia. Ann. N Y Acad. Sci. 1157, 61-70. doi: 10.1111/j.1749-6632.2008.04119.x

Purdon, P. L., Pierce, E. T., Mukamel, E. A., Prerau, M. J., Walsh, J. L., Wong, K. F., et al. (2013). Electroencephalogram signatures of loss and recovery of consciousness from propofol. Proc. Natl. Acad. Sci. US A 110, E1142-E1151. doi: 10.1073/pnas.1221180110

Purdon, P. L., Sampson, A., Pavone, K. J., and Brown, E. N. (2015). Clinical electroencephalography for anesthesiologists: part I: background and basic signatures. Anesthesiology 123, 937-960. doi: 10.1097/ALN.0000000000000841

Putnam, R. W., Filosa, J. A., and Ritucci, N. A. (2004). Cellular mechanisms involved in $\mathrm{CO}_{2}$ and acid signaling in chemosensitive neurons. Am. J. Physiol. Cell Physiol. 287, C1493-C1526. doi: 10.1152/ajpcell.00282.2004

Quasha, A. L., Eger, E. I. II., and Tinker, J. H. (1980). Determination and applications of MAC. Anesthesiology 53, 315-334. doi: 10.1097/00000542198010000-00008

Raichle, M. E. (2015). The brain's default mode network. Annu. Rev. Neurosci. 38, 433-447. doi: 10.1146/annurev-neuro-071013-014030

Raichle, M. E., Macleod, A. M., Snyder, A. Z., Powers, W. J., Gusnard, D. A., and Shulman, G. L. (2001). A default mode of brain function. Proc. Natl. Acad. Sci. U S A 98, 676-682. doi: 10.1073/pnas.98.2.676

Ranft, A., Golkowski, D., Kiel, T., Riedl, V., Kohl, P., Rohrer, G., et al. (2016). Neural correlates of sevoflurane-induced unconsciousness identified by simultaneous functional magnetic resonance imaging and electroencephalography. Anesthesiology 125, 861-872. doi: 10.1097/ALN. 0000000000001322

Raper, A. J., and Levasseur, J. E. (1971). Accurate sustained measurement of intra-luminal pressure from the microvasculature. Cardiovasc. Res. 5, 589-593. doi: $10.1093 / \mathrm{cvr} / 5.4 .589$

Raz, A., Grady, S. M., Krause, B. M., Uhlrich, D. J., Manning, K. A., and Banks, M. I. (2014). Preferential effect of isoflurane on top-down vs. bottom-up pathways in sensory cortex. Front. Syst. Neurosci. 8:191. doi: 10.3389/fnsys.2014.00191

Redinbaugh, M. J., Phillips, J. M., Kambi, N. A., Mohanta, S., Andryk, S., Dooley, G. L., et al. (2020). Thalamus modulates consciousness via layerspecific control of cortex. Neuron doi: 10.1016/j.neuron.2020.01.005 [Epub ahead of print].

Rees, G., Kreiman, G., and Koch, C. (2002). Neural correlates of consciousness in humans. Nat. Rev. Neurosci. 3, 261-270. doi: 10.1038/nrn783

Reig, R., Mattia, M., Compte, A., Belmonte, C., and Sanchez-Vives, M. V. (2010). Temperature modulation of slow and fast cortical rhythms. J. Neurophysiol. 103, 1253-1261. doi: 10.1152/jn.00890.2009

Reimann, H. M., Hentschel, J., Marek, J., Huelnhagen, T., Todiras, M., Kox, S., et al. (2016). Normothermic mouse functional MRI of acute focal thermostimulation for probing nociception. Sci. Rep. 6:17230. doi: 10.1038/ srep 17230

Reimann, H. M., Todiras, M., Hodge, R., Huelnhagen, T., Millward, J. M., Turner, R., et al. (2018). Somatosensory BOLD fMRI reveals close link between salient blood pressure changes and the murine neuromatrix. NeuroImage 172, 562-574. doi: 10.1016/j.neuroimage.2018.02.002

Reimer, J., Mcginley, M. J., Liu, Y., Rodenkirch, C., Wang, Q., Mccormick, D. A., et al. (2016). Pupil fluctuations track rapid changes in adrenergic and cholinergic activity in cortex. Nat. Commun. 7:13289. doi: $10.1038 /$ ncomms 13289
Roberts, J. A., Gollo, L. L., Abeysuriya, R. G., Roberts, G., Mitchell, P. B., Woolrich, M. W., et al. (2019). Metastable brain waves. Nat. Commun. 10:1056. doi: 10.1038/s41467-019-08999-0

Roth, B. L. (2016). DREADDs for neuroscientists. Neuron 89, 683-694. doi: 10.1016/j.neuron.2016.01.040

Rubin, R., Abbott, L. F., and Sompolinsky, H. (2017). Balanced excitation and inhibition are required for high-capacity, noise-robust neuronal selectivity. Proc. Natl. Acad. Sci. U S A 114, E9366-E9375. doi: 10.1073/pnas.1705841114

Ruiz-Mejias, M., Ciria-Suarez, L., Mattia, M., and Sanchez-Vives, M. V. (2011). Slow and fast rhythms generated in the cerebral cortex of the anesthetized mouse. J. Neurophysiol. 106, 2910-2921. doi: 10.1152/jn.00440.2011

Ryali, S., Shih, Y.-Y. I., Chen, T., Kochalka, J., Albaugh, D., Fang, Z., et al (2016). Combining optogenetic stimulation and fMRI to validate a multivariate dynamical systems model for estimating causal brain interactions. NeuroImage 132, 398-405. doi: 10.1016/j.neuroimage.2016.02.067

Saalmann, Y. B. (2014). Intralaminar and medial thalamic influence on cortical synchrony, information transmission and cognition. Front. Syst. Neurosci. 8:83. doi: 10.3389/fnsys.2014.00083

Saalmann, Y. B., Pinsk, M. A., Wang, L., Li, X., and Kastner, S. (2012). The pulvinar regulates information transmission between cortical areas based on attention demands. Science 337, 753-756. doi: 10.1126/science. 1223082

Sachidhanandam, S., Sreenivasan, V., Kyriakatos, A., Kremer, Y., and Petersen, C. C. H. (2013). Membrane potential correlates of sensory perception in mouse barrel cortex. Nat. Neurosci. 16, 1671-1677. doi: 10.1038/ nn.3532

Sadaghiani, S., Scheeringa, R., Lehongre, K., Morillon, B., Giraud, A. L., D'Esposito, M., et al. (2012). $\alpha$-band phase synchrony is related to activity in the fronto-parietal adaptive control network. J. Neurosci. 32, 14305-14310. doi: 10.1523/jneurosci.1358-12.2012

Samuels, E. R., and Szabadi, E. (2008a). Functional neuroanatomy of the noradrenergic locus coeruleus: its roles in the regulation of arousal and autonomic function part I: principles of functional organisation. Curr. Neuropharmacol. 6, 235-253. doi: 10.2174/157015908785777229

Samuels, E. R., and Szabadi, E. (2008b). Functional neuroanatomy of the noradrenergic locus coeruleus: its roles in the regulation of arousal and autonomic function part II: physiological and pharmacological manipulations and pathological alterations of locus coeruleus activity in humans. Curr. Neuropharmacol. 6, 254-285. doi: 10.2174/157015908785777193

Sanchez-Vives, M. V., Massimini, M., and Mattia, M. (2017). Shaping the default activity pattern of the cortical network. Neuron 94, 993-1001. doi: 10.1016/j. neuron.2017.05.015

Sanders, T. H. (2017). Stimulation of cortico-subthalamic projections amplifies resting motor circuit activity and leads to increased locomotion in dopaminedepleted mice. Front. Integr. Neurosci. 11:24. doi: 10.3389/fnint.2017. 00024

Sanders, R. D., Banks, M. I., Darracq, M., Moran, R., Sleigh, J., Gosseries, O., et al. (2018). Propofol-induced unresponsiveness is associated with impaired feedforward connectivity in cortical hierarchy. Br. J. Anaesth. 121, 1084-1096. doi: 10.1016/j.bja.2018.07.006

Sanders, R. D., Degos, V., and Young, W. L. (2011). Cerebral perfusion under pressure: is the autoregulatory "plateau" a level playing field for all? Anaesthesia 66, 968-972. doi: 10.1111/j.1365-2044.2011.06915.x

Sanders, R., Tononi, G., Laureys, S., and Sleigh, J. (2012). Unresponsiveness $\neq$ unconsciousness. Anesthesiology 116, 946-959. doi: 10.1097/ALN. 0b013e318249d0a7

Saper, C. B., Fuller, P. M., Pedersen, N. P., Lu, J., and Scammell, T. E. (2010). Sleep state switching. Neuron 68, 1023-1042. doi: 10.1016/j.neuron.2010. 11.032

Saper, C. B., Scammell, T. E., and Lu, J. (2005). Hypothalamic regulation of sleep and circadian rhythms. Nature 437, 1257-1263. doi: 10.1038/ nature 04284

Sarasso, S., Boly, M., Napolitani, M., Gosseries, O., Charland-Verville, V., Casarotto, S., et al. (2015). Consciousness and complexity during unresponsiveness induced by propofol, xenon, and ketamine. Curr. Biol. 25, 3099-3105. doi: 10.1016/j.cub.2015.10.014

Scammell, T. E., Arrigoni, E., and Lipton, J. O. (2017). Neural circuitry of wakefulness and sleep. Neuron 93, 747-765. doi: 10.1016/j.neuron.2017.01.014 
Schlegel, F. (2017). Improving Mouse FMRI and Our Understanding of Its Underpinnings. [Dissertation] Zürich, Switzerland: ETH Zurich.

Schlegel, F., Schroeter, A., and Rudin, M. (2015). The hemodynamic response to somatosensory stimulation in mice depends on the anesthetic used: implications on analysis of mouse fMRI data. NeuroImage 116, 40-49. doi: 10.1016/j.neuroimage.2015.05.013

Schlegel, F., Sych, Y., Schroeter, A., Stobart, J., Weber, B., Helmchen, F., et al. (2018). Fiber-optic implant for simultaneous fluorescence-based calcium recordings and BOLD fMRI in mice. Nat. Protoc. 13, 840-855. doi: $10.1038 /$ nprot. 2018.003

Schmid, F., Wachsmuth, L., Schwalm, M., Prouvot, P. H., Jubal, E. R., Fois, C., et al. (2016). Assessing sensory versus optogenetic network activation by combining (o)fMRI with optical $\mathrm{Ca}^{2+}$ recordings. J. Cereb. Blood Flow Metab. 36, 1885-1900. doi: 10.1177/0271678x15619428

Schneider, D. W., and Logan, G. D. (2014). Modelling response selection in task switching: testing the contingent encoding assumption. Q. J. Exp. Psychol. 67, 1074-1095. doi: 10.1080/17470218.2013.843009

Schölvinck, M. L., Saleem, A. B., Benucci, A., Harris, K. D., and Carandini, M. (2015). Cortical state determines global variability and correlations in visual cortex. J. Neurosci. 35, 170-178. doi: 10.1523/jneurosci.499413.2015

Schroeder, C. E., and Lakatos, P. (2009). Low-frequency neuronal oscillations as instruments of sensory selection. Trends in Neurosci. 32, 9-18. doi: 10.1016/j. tins.2008.09.012

Schroeder, K. E., Irwin, Z. T., Gaidica, M., Nicole Bentley, J., Patil, P. G., Mashour, G. A., et al. (2016). Disruption of corticocortical information transfer during ketamine anesthesia in the primate brain. NeuroImage 134, 459-465. doi: 10.1016/j.neuroimage.2016.04.039

Schroeter, A., Grandjean, J., Schlegel, F., Saab, B. J., and Rudin, M. (2017). Contributions of structural connectivity and cerebrovascular parameters to functional magnetic resonance imaging signals in mice at rest and during sensory paw stimulation. J. Cereb. Blood Flow Metab. 37, 2368-2382. doi: $10.1177 / 0271678 \times 16666292$

Schroeter, A., Schlegel, F., Seuwen, A., Grandjean, J., and Rudin, M. (2014). Specificity of stimulus-evoked fMRI responses in the mouse: the influence of systemic physiological changes associated with innocuous stimulation under four different anesthetics. NeuroImage 94, 372-384. doi: 10.1016/j.neuroimage. 2014.01.046

Schröter, M. S., Spoormaker, V. I., Schorer, A., Wohlschlager, A., Czisch, M., Kochs, E. F., et al. (2012). Spatiotemporal reconfiguration of largescale brain functional networks during propofol-induced loss of consciousness. J. Neurosci. 32, 12832-12840. doi: 10.1523/jneurosci.604611.2012

Schumacher, J. W., Schneider, D. M., and Woolley, S. M. N. (2011). Anesthetic state modulates excitability but not spectral tuning or neural discrimination in single auditory midbrain neurons. Neuroscience 106, 500-514. doi: 10.1152/jn. 01072.2010

Schwalm, M., and Easton, C. (2016). Cortical temperature change: a tool for modulating brain states? eNeuro 3:ENEURO.0096-16.2016. doi: 10.1523/eneuro.0096-16.2016

Schwalm, M., Schmid, F., Wachsmuth, L., Backhaus, H., Kronfeld, A., Jury, F. A., et al. (2017). Cortex-wide BOLD fMRI activity reflects locally-recorded slow oscillation-associated calcium waves. Elife 6:e27602. doi: 10.7554/elife. 27602

Schwertner, A., Zortea, M., Torres, F. V., and Caumo, W. (2018). Effects of subanesthetic ketamine administration on visual and auditory event-related potentials (ERP) in humans: a systematic review. Front. Behav. Neurosci. 12:70. doi: $10.3389 /$ fnbeh.2018.00070

Seamans, J. (2008). Losing inhibition with ketamine. Nat. Chem. Biol. 4, 91-93. doi: 10.1038/nchembio0208-91

Sellers, K. K., Bennett, D. V., Hutt, A., Williams, J. H., and Fröhlich, F. (2015). Awake vs. anesthetized: layer-specific sensory processing in visual cortex and functional connectivity between cortical areas. J. Neurophysiol. 113, 3798-3815. doi: 10.1152/jn.00923.2014

Senzai, Y., Fernandez-Ruiz, A., and Buzsáki, G. (2019). Layer-specific physiological features and interlaminar interactions in the primary visual cortex of the mouse. Neuron 101, 500.e5-513.e5. doi: 10.1016/j.neuron.2018. 12.009
Seth, A. K., Baars, B. J., and Edelman, D. B. (2005). Criteria for consciousness in humans and other mammals. Conscious. Cogn. 14, 119-139. doi: 10.1016/j. concog.2004.08.006

Seth, A. K., and Tsakiris, M. (2018). Being a beast machine: the somatic basis of selfhood. Trends Cogn. Sci. 22, 969-981. doi: 10.1016/j.tics.2018.08.008

Sforazzini, F., Schwarz, A. J., Galbusera, A., Bifone, A., and Gozzi, A. (2014). Distributed BOLD and CBV-weighted resting-state networks in the mouse brain. NeuroImage 87, 403-415. doi: 10.1016/j.neuroimage.2013.09.050

Shah, D., Blockx, I., Guns, P., Paul, P., Deyn, D., Van Dam, D., et al. (2015). Acute modulation of the cholinergic system in the mouse brain detected by pharmacological resting-state functional MRI. NeuroImage 109, 151-159. doi: 10.1016/j.neuroimage.2015.01.009

Shah, D., Jonckers, E., Praet, J., Vanhoutte, G., Delgado, Y. P., Bigot, C., et al. (2013). Resting state FMRI reveals diminished functional connectivity in a mouse model of amyloidosis. PLoS One 8:e84241. doi: 10.1371/journal.pone. 0084241

Sharp, P. S., Shaw, K., Boorman, L., Harris, S., Kennerley, A. J., Azzouz, M., et al. (2015). Comparison of stimulus-evoked cerebral hemodynamics in the awake mouse and under a novel anesthetic regime. Sci. Rep. 5:14890. doi: 10.1038/srep14890

Shein-Idelson, M., Ondracek, J. M., Liaw, H. P., Reiter, S., and Laurent, G. (2016). Slow waves, sharp waves, ripples, and REM in sleeping dragons. Science 352, 590-595. doi: 10.1126/science.aaf3621

Shelton, L., Becerra, L., and Borsook, D. (2012a). Unmasking the mysteries of the habenula in pain and analgesia. Prog. Neurobiol. 96, 208-219. doi: 10.1016/j. pneurobio.2012.01.004

Shelton, L., Pendse, G., Maleki, N., Moulton, E. A., Lebel, A., Becerra, L., et al. (2012b). Mapping pain activation and connectivity of the human habenula. J. Neurophysiol. 107, 2633-2648. doi: 10.1152/jn.00012.2012

Sherin, J. E., Elmquist, J. K., Torrealba, F., and Saper, C. B. (1998). Innervation of histaminergic tuberomammillary neurons by GABAergic and galaninergic neurons in the ventrolateral preoptic nucleus of the rat. J. Neurosci. 18, 4705-4721. doi: 10.1523/jneurosci.18-12-04705.1998

Sherman, S. M. (2017). Functioning of circuits connecting thalamus and cortex. Compr. Physiol. 7, 713-739. doi: 10.1002/cphy.c160032

Sheroziya, M., and Timofeev, I. (2014). Global intracellular slow-wave dynamics of the thalamocortical system. J. Neurosci. 34, 8875-8893. doi: 10.1523/jneurosci. 4460-13.2014

Sheroziya, M., and Timofeev, I. (2015). Moderate cortical cooling eliminates thalamocortical silent states during slow oscillation. J. Neurosci. 35, 13006-13019. doi: 10.1523/jneurosci.1359-15.2015

Shim, H.-J., Jung, W. B., Schlegel, F., Lee, J., Kim, S., Lee, J., et al. (2018). Mouse fMRI under ketamine and xylazine anesthesia: robust contralateral somatosensory cortex activation in response to forepaw stimulation. NeuroImage 177, 30-44. doi: 10.1016/j.neuroimage.2018. 04.062

Shimaoka, D., Harris, K. D., and Carandini, M. (2018). Effects of arousal on mouse sensory cortex depend on modality. Cell Rep. 22, 3160-3167. doi: 10.1016/j. celrep.2018.02.092

Shimosegawa, E., Kanno, I., Hatazawa, J., Fujita, H., Iida, H., Miura, S., et al. (1995). Photic stimulation study of changing the arterial partial pressure level of carbon dioxide. J. Cereb. Blood Flow Metab. 15, 111-114. doi: 10.1038/jcbfm. 1995.12

Shofty, B., Bergmann, E., Zur, G., Asleh, J., Bosak, N., Kavushansky, A., et al. (2019). Autism-associated Nf1 deficiency disrupts corticocortical and corticostriatal functional connectivity in human and mouse. Neurobiol. Dis. 130:104479. doi: 10.1016/j.nbd.2019.104479

Sikkens, T., Bosman, C. A., and Olcese, U. (2019). The role of top-down modulation in shaping sensory processing across brain states: implications for consciousness. Front. Syst. Neurosci. 13:31. doi: 10.3389/fnsys.2019. 00031

Sinclair, M. D. (2003). A review of the physiological effects of $\alpha 2$-agonists related to the clinical use of medetomidine in small animal practice. Can. Vet. J. 44, 885-897.

Sinclair, D. R., Chung, F., and Smiley, A. (2003). General anesthesia does not impair simulator driving skills in volunteers in the immediate recovery period-a pilot study. Can. J. Anaesth. 50, 238-245. doi: 10.1007/bf03 017791 
Sinner, B., and Graf, B. M. (2008). Ketamine. Handb. Exp. Pharmacol. 182, 313-333. doi: 10.1007/978-3-540-74806-9_15

Sinning, A., and Hübner, C. A. (2013). Minireview: $\mathrm{pH}$ and synaptic transmission. FEBS Lett. 587, 1923-1928. doi: 10.1016/j.febslet.2013.04.045

Sirmpilatze, N., Baudewig, J., and Boretius, S. (2019). Temporal stability of fMRI in medetomidine-anesthetized rats. Sci. Rep. 9:16673. doi: 10.1038/s41598-01953144-y

Sirota, A., Csicsvari, J., Buhl, D., and Buzsáki, G. (2003). Communication between neocortex and hippocampus during sleep in rodents. Proc. Natl. Acad. Sci. U S A 100, 2065-2069. doi: 10.1073/pnas.0437938100

Sleigh, J., Warnaby, C., and Tracey, I. (2018). General anaesthesia as fragmentation of selfhood: insights from electroencephalography and neuroimaging. $\mathrm{Br}$. J. Anaesth. 121, 233-240. doi: 10.1016/j.bja.2017.12.038

Slézia, A., Hangya, B., Ulbert, I., and Acsády, L. (2011). Phase advancement and nucleus-specific timing of thalamocortical activity during slow cortical oscillation. J. Neurosci. 31, 607-617. doi: 10.1523/jneurosci.337510.2011

Solt, K., Van Dort, C. J., Chemali, J. J., Taylor, N. E., Kenny, J. D., and Brown, E. N. (2014). Electrical stimulation of the ventral tegmental area induces reanimation from general anesthesia. Anesthesiology 121, 311-319. doi: 10.1097/ALN. 0000000000000117

Song, A. H., Kucyi, A., Napadow, V., Brown, E. N., Loggia, M. L., and Akeju, O. (2017). Pharmacological modulation of noradrenergic arousal circuitry disrupts functional connectivity of the locus ceruleus in humans. J. Neurosci. 37, 6938-6945. doi: 10.1523/jneurosci.0446-17.2017

Song, B., Ma, N., Liu, G., Zhang, H., Yu, L., Liu, L., et al. (2019). Maximal flexibility in dynamic functional connectivity with critical dynamics revealed by fMRI data analysis and brain network modelling. J. Neural Eng. 16:056002. doi: 10.1088/1741-2552/ab20bc

Soltesz, I., Lightowler, S., Leresche, N., Jassik-Gerschenfeld, D., Pollard, C. E., and Crunelli, V. (1991). Two inward currents and the transformation of low frequency oscillations of rat and cat thalamocortical cells. J. Physiol. 441, 175-197. doi: 10.1113/jphysiol.1991.sp018745

Stafford, J. M., Jarrett, B. R., Miranda-Dominguez, O., Mills, B. D., Cain, N., Mihalas, S., et al. (2014). Large-scale topology and the default mode network in the mouse connectome. Proc. Natl. Acad. Sci. U S A 111, 18745-18750. doi: $10.1073 /$ pnas. 1404346111

Steffey, E. P. (2017). Methodology for determining minimum alveolar concentration: a critical appraisal. Vet. Anaesth. Analg. 44, 2-6. doi: 10.1016/j. vaa.2016.12.001

Steriade, M., Curró Dossi, R., and Nunez, A. (1991). Network modulation of a slow intrinsic oscillation of cat thalamocortical neurons implicated in sleep delta waves: cortical potentiation and brainstem cholinergic suppression. J. Neurosci. 11, 3200-3217. doi: 10.1523/jneurosci.11-10-03200.1991

Steriade, M. (2000). Corticothalamic resonance, states of vigilance and mentation. Neuroscience 101, 243-276. doi: 10.1016/s0306-4522(00)00353-5

Steriade, M. (2001). Impact of network activities on neuronal properties in corticothalamic systems. J. Neurophysiol. 86, 1-39. doi: 10.1152/jn.2001. 86.1.1

Steriade, M., Amzica, F., and Contreras, D. (1994). Cortical and thalamic cellular correlates of electroencephalographic burst-suppression. Electroencephalogr. Clin. Neurophysiol. 90, 1-16. doi: 10.1016/0013-4694(94) 90108-2

Steriade, M., Amzica, F., and Nufiez, A. (1993a). Cholinergic and noradrenergic modulation of the slow (approximately $0.3 \mathrm{~Hz}$ ) oscillation in neocortical cells. J. Neurophysiol. 70, 1385-1400. doi: 10.1152/jn.1993.70.4.1385

Steriade, M., Contreras, D., Curró Dossi, R., and Nuñez, A. (1993b). The slow $(<1 \mathrm{~Hz})$ oscillation in reticular thalamic and thalamocortical neurons: scenario of sleep rhythm generation in interacting thalamic and neocortical networks. J. Neurosci. 13, 3284-3299. doi: 10.1523/jneurosci.13-0803284.1993

Steriade, M., Nuñez, A., and Amzica, F. (1993c). Intracellular analysis of relations between the slow $(<1 \mathrm{~Hz})$ neocortical oscillation and other sleep rhythms of the electroencephalogram. J. Neurosci. 13, 3266-3283. doi: 10.1523/jneurosci. 13-08-03266.1993

Steriade, M., Nuñez, A., and Amzica, F. (1993d). A novel slow $(<1 \mathrm{~Hz})$ oscillation of neocortical neurons in vivo: depolarizing and hyperpolarizing components. J. Neurosci. 13, 3252-3265. doi: 10.1523/jneurosci.13-0803252.1993

Steriade, M., and Contreras, D. (1995). Relations between cortical and thalamic cellular events during transition from sleep pattern to paroxysmal activity. J. Neurosci. 15, 623-642. doi: 10.1523/jneurosci.15-01-00623.1995

Steriade, M., Deschenes, M., Domich, L., and Mulle, C. (1985). Abolition of spindle oscillations in thalamic neurons disconnected from nucleus reticularis thalami. J. Neurophysiol. 54, 1473-1497. doi: 10.1152/jn.1985.54.6.1473

Steriade, M., Domich, L., Oakson, G., and Deschênes, M. (1987). The deafferented reticularis thalami nucleus generates spindle rhythmicity. J. Neurophysiol. 57 , 260-273. doi: 10.1152/jn.1987.57.1.260

Steriade, M., Timofeev, I., and Grenier, F. (2001). Natural waking and sleep states: a view from inside neocortical neurons. J. Neurophysiol. 85, 1969-1985. doi: 10.1152/jn.2001.85.5.1969

Storm, J. F., Boly, M., Casali, A. G., Massimini, M., Olcese, U., Pennartz, C. M. A., et al. (2017). Consciousness regained: disentangling mechanisms, brain systems and behavioral responses. J. Neurosci. 37, 10882-10893. doi: 10.1523/jneurosci. 1838-17.2017

Straathof, M., Sinke, M. R., Dijkhuizen, R. M., and Otte, W. M. (2019). A systematic review on the quantitative relationship between structural and functional network connectivity strength in mammalian brains. J. Cereb. Blood Flow Metab. 39, 189-209. doi: 10.1177/0271678x18809547

Stroh, A., Adelsberger, H., Groh, A., Rühlmann, C., Fischer, S., Schierloh, A., et al. (2013). Making waves: initiation and propagation of corticothalamic $\mathrm{Ca}^{2+}$ waves in vivo. Neuron 77, 1136-1150. doi: 10.1016/j.neuron.2013. 01.031

Sumiyoshi, A., Keeley, R. J., and Lu, H. (2019). Physiological considerations of functional magnetic resonance imaging in animal models. Biol. Psychiatry Cogn. Neurosci. Neuroimaging 4, 522-532. doi: 10.1016/j.bpsc.2018. 08.002

Sun, Y. M., Favre, I., Schild, L., and Moczydlowski, E. (1997). On the structural basis for size-selective permeation of organic cations through the voltagegated sodium channel. Effect of alanine mutations at the DEKA locus on selectivity, inhibition by $\mathrm{Ca}^{2+}$ and $\mathrm{H}^{+}$and molecular sieving. J. Gen. Physiol. 110, 693-715. doi: 10.1085/jgp.110.6.693

Sun, W., McConnell, E., Pare, J. F., Xu, Q., Chen, M., Peng, W., et al. (2013). Glutamate-dependent neuroglial calcium signaling differs between young and adult brain. Science 339, 197-200. doi: 10.1126/science.1226740

Sun, Y. G., Wu, C. S., Renger, J. J., Uebele, V. N., Lu, H. C., and Beierlein, M. (2012). GABAergic synaptic transmission triggers action potentials in thalamic reticular nucleus neurons. J. Neurosci. 32, 7782-7790. doi: 10.1523/jneurosci. 0839-12.2012

Sunkin, S. M., Ng, L., Lau, C., Dolbeare, T., Gilbert, T. L., Thompson, C. L., et al. (2013). Allen brain atlas: an integrated spatio-temporal portal for exploring the central nervous system. Nucleic Acids Res. 41, D996-D1008. doi: $10.1093 /$ nar/gks1042

Suzuki, M., and Larkum, M. E. (2020). General anesthesia decouples cortical pyramidal neurons. Cell 180, 666.e13-676.e13. doi: 10.1016/j.cell.2020. 01.024

Takuwa, H., Matsuura, T., Obata, T., Kawaguchi, H., Kanno, I., and Ito, H. (2012). Hemodynamic changes during somatosensory stimulation in awake and isoflurane-anesthetized mice measured by laser-Doppler flowmetry. Brain Res. 1472, 107-112. doi: 10.1016/j.brainres.2012.06.049

Taylor, N. E., Van Dort, C. J., Kenny, J. D., Pei, J., Guidera, J. A., Vlasov, K. Y., et al. (2016). Optogenetic activation of dopamine neurons in the ventral tegmental area induces reanimation from general anesthesia. Proc. Natl. Acad. Sci. U S A 113, 12826-12831. doi: 10.1073/pnas.1614340113

Theyel, B. B., Llano, D. A., and Sherman, S. M. (2010). The corticothalamocortical circuit drives higher-order cortex in the mouse. Nat. Neurosci. 13, 84-88. doi: $10.1038 / \mathrm{nn} .2449$

Thompson, G. J., Merritt, M. D., Pan, W. J., Magnuson, M. E., Grooms, J. K., Jaeger, D., et al. (2013). Neural correlates of time-varying functional connectivity in the rat. NeuroImage $83,826-836$. doi: $10.1016 /$ j.neuroimage. 2013.07.036

Timofeev, I., Grenier, F., Bazhenov, M., Sejnowski, T. J., and Steriade, M. (2000). Origin of slow cortical oscillations in deafferented cortical slabs. Cereb. Cortex 10, 1185-1199. doi: $10.1093 /$ cercor/10.12.1185 
Tombaugh, G. C., and Somjen, G. G. (1996). Effects of extracellular pH on voltagegated $\mathrm{Na}^{+}, \mathrm{K}^{+}$and $\mathrm{Ca}^{2+}$ currents in isolated rat CA1 neurons. J. Physiol. 493, 719-732. doi: 10.1113/jphysiol.1996.sp021417

Tononi, G., Boly, M., Massimini, M., and Koch, C. (2016). Integrated information theory: from consciousness to its physical substrate. Nat. Rev. Neurosci. 17, 450-461. doi: 10.1038/nrn.2016.44

Toussay, X., Basu, K., Lacoste, B., and Hamel, E. (2013). Locus coeruleus stimulation recruits a broad cortical neuronal network and increases cortical perfusion. J. Neurosci. 33, 3390-3401. doi: 10.1523/jneurosci.334612.2013

Tracey, I. (2017). Neuroimaging mechanisms in pain: from discovery to translation. Pain 158, S115-S122. doi: 10.1097/j.pain.0000000000000863

Trageser, J. C., Burke, K. A., Masri, R., Li, Y., Sellers, L., and Keller, A. (2006). State-dependent gating of sensory inputs by zona incerta. J. Neurophysiol. 96, 1456-1463. doi: 10.1152/jn.00423.2006

Uchida, S., and Kagitani, F. (2018). Effect of basal forebrain stimulation on extracellular acetylcholine release and blood flow in the olfactory bulb. J. Physiol. Sci. 68, 415-423. doi: 10.1007/s12576-017-0542-z

Uhrig, L., Sitt, J. D., Jacob, A., Tasserie, J., Barttfeld, P., Dupont, M., et al. (2018). Resting-state dynamics as a cortical signature of anesthesia in monkeys. Anesthesiology 129, 942-958. doi: 10.1097/aln.00000000000 02336

Ulrich-Lai, Y. M., and Herman, J. P. (2009). Neural regulation of endocrine and autonomic stress responses. Nat. Rev. Neurosci. 10, 397-409. doi: $10.1038 / \mathrm{nrn} 2647$

van Alst, T. M., Wachsmuth, L., Datunashvili, M., Albers, F., Just, N., Budde, T., et al. (2019). Anesthesia differentially modulates neuronal and vascular contributions to the BOLD signal. NeuroImage 195, 89-103. doi: 10.1016/j. neuroimage.2019.03.057

Van Bockstaele, E. J., Saunders, A., Telegan, P., and Page, M. E. (1999). Localization of mu-opioid receptors to locus coeruleus-projecting neurons in the rostral medulla: morphological substrates and synaptic organization. Synapse 34, 154-167. doi: 10.1002/(SICI)1098-2396(199911)34:2<154::AIDSYN8>3.0.CO;2-C

Van de Ville, D., Britz, J., and Michel, C. M. (2010). EEG microstate sequences in healthy humans at rest reveal scale-free dynamics. Proc. Natl. Acad. Sci. U S A 107, 18179-18184. doi: 10.1073/pnas. 1007841107

van Kerkoerle, T., Self, M. W., Dagnino, B., Gariel-Mathis, M. A., Poort, J., van der Togt, C., et al. (2014). Alpha and gamma oscillations char- acterize feedback and feedforward processing in monkey visual cortex. Proc. Natl. Acad. Sci. U S A 111, 14332-14341. doi: 10.1073/pnas.1402773111

Varela, C. (2014). Thalamic neuromodulation and its implications for executive networks. Front. Neural Circuits 8:69. doi: 10.3389/fncir.2014.00069

Vazey, E. M., and Aston-Jones, G. (2014). Designer receptor manipulations reveal a role of the locus coeruleus noradrenergic system in isoflurane general anesthesia. Proc. Natl. Acad. Sci. U S A 111, 3859-3864. doi: 10.1073/pnas. 1310025111

Venn, R. M., and Grounds, R. M. (2001). Comparison between dexmedetomidine and propofol for sedation in the intensive care unit: patient and clinician perceptions. Br. J. Anaesth. 87, 684-690. doi: 10.1093/bja/87.5.684

Ventura-Antunes, L., Mota, B., and Herculano-Houzel, S. (2013). Different scaling of white matter volume, cortical connectivity and gyrification across rodent and primate brains. Front. Neuroanat. 7:3. doi: 10.3389/fnana.2013. 00003

Veselis, R. A., Feshchenko, V. A., Reinsel, R. A., Beattie, B., and Akhurst, T. J. (2005). Propofol and thiopental do not interfere with regional cerebral blood flow response at sedative concentrations. Anesthesiology 102, 26-34. doi: 10.1097/00000542-200501000-00008

Vetter, I., Touska, F., Hess, A., Hinsbey, R., Sattler, S., Lampert, A., et al. (2012). Ciguatoxins activate specific cold pain pathways to elicit burning pain from cooling. EMBO J. 3, 3795-3808. doi: 10.1038/emboj.2012.207

Vijayan, S., Ching, S., Purdon, P. L., Brown, E. N., and Kopell, N. J. (2013). Thalamocortical mechanisms for the anteriorization of $\alpha$ rhythms during propofol-induced unconsciousness. J. Neurosci. 33, 11070-11075. doi: 10.1523/jneurosci.5670-12.2013

Vincent, J. L., Patel, G. H., Fox, M. D., Snyder, A. Z., Baker, J. T., Van Essen, D. C., et al. (2007). Intrinsic functional architecture in the anaesthetized monkey brain. Nature 447, 83-86. doi: 10.1038/nature05758
Vizuete, J. A., Pillay, S., Ropella, K. M., and Hudetz, A. G. (2014). Graded defragmentation of cortical neuronal firing during recovery of consciousness in rats. Neuroscience 275, 340-351. doi: 10.1016/j.neuroscience.2014.06.018

Vlisides, P. E., Bel-Bahar, T., Lee, U., Li, D., Kim, H., Janke, E., et al. (2017). Neurophysiologic correlates of ketamine sedation and anesthesia: a high-density electroencephalography study in healthy volunteers. Anesthesiology 127, 58-69. doi: 10.1097/aln.0000000000001671

Vogt, B. A., Hof, P. R., Friedman, D. P., Sikes, R. W., and Vogt, L. J. (2008). Norepinephrinergic afferents and cytology of the macaque monkey midline, mediodorsal and intralaminar thalamic nuclei. Brain Struct. Funct. 212, 465-479. doi: 10.1007/s00429-008-0178-0

Vogt, B. A., and Pandya, D. N. (1987). Cingulate cortex of the rhesus monkey: II. Cortical afferents. J. Comp. Neurol. 262, 271-289. doi: 10.1002/cne.9026 20208

Volgushev, M., Vidyasagar, T. R., Chistiakova, M., Yousef, T., and Eysel, U. T. (2000). Membrane properties and spike generation in rat visual cortical cells during reversible cooling. J. Physiol. 522, 59-76. doi: 10.1111/j.1469-7793.2000. 0059m.x

Voss, B., Thienel, R., Reske, M., Kellermann, T., Sheldrick, A. J., Halfter, S., et al. (2012). Cholinergic blockade under working memory demands encountered by increased rehearsal strategies: evidence from fMRI in healthy subjects. Eur. Arch. Psychiatry Clin. Neurosci. 262, 329-339. doi: 10.1007/s00406-0110267-6

Vyazovskiy, V. V., Olcese, U., Hanlon, E. C., Nir, Y., Cirelli, C., and Tononi, G. (2011). Local sleep in awake rats. Nature 472, 443-447. doi: 10.1038/nature10009

Wager, T. D., Atlas, L. Y., Lindquist, M. A., Roy, M., Woo, C. W., and Kross, E. (2013). An fMRI-based neurologic signature of physical pain. N. Engl. J. Med. 368, 1388-1397. doi: 10.1056/NEJMoa1204471

Wang, R., Foniok, T., Wamsteeker, J. I., Qiao, M., Tomanek, B., Vivanco, R. A., et al. (2006). Transient blood pressure changes affect the functional magnetic resonance imaging detection of cerebral activation. NeuroImage 31, 1-11. doi: 10.1016/j.neuroimage.2005.12.004

Wang, R., Macmillan, L. B., Fremeau, R. T. Jr., Magnuson, M. A., Lindner, J., and Limbird, L. E. (1996). Expression of $\alpha 2$-adrenergic receptor subtypes in the mouse brain: evaluation of spatial and temporal information imparted by $3 \mathrm{~kb}$ of 5 / regulatory sequence for the $\alpha 2 \mathrm{~A}$ AR-receptor gene in transgenic animals. Neuroscience 74, 199-218. doi: 10.1016/0306-4522(96) 00116-9

Warnaby, C. E., Seretny, M., Ni Mhuircheartaigh, R., Rogers, R., Jbabdi, S., Sleigh, J., et al. (2016). Anesthesia-induced suppression of human dorsal anterior insula responsivity at loss of volitional behavioral response. Anesthesiology 124, 766-778. doi: 10.1097/aln.0000000000001027

Weber, F., and Dan, Y. (2016). Circuit-based interrogation of sleep control. Nature 538, 51-59. doi: 10.1038/nature19773

Wilding, L. A., Hampel, J. A., Khoury, B. M., Kang, S., Machado-Aranda, D., Raghavendran, K., et al. (2017). Benefits of $21 \%$ oxygen compared with $100 \%$ oxygen for delivery of isoflurane to mice (Mus musculus) and rats (Rattus norvegicus). J. Am. Assoc. Lab. Anim. Sci. 56, 148-154.

Williams, R. H., Jensen, L. T., Verkhratsky, A., Fugger, L., and Burdakov, D. (2007). Control of hypothalamic orexin neurons by acid and CO2. Proc. Natl. Acad. Sci. US A 104, 10685-10690. doi: 10.1073/pnas.0702676104

Wimmer, R. D., Astori, S., Bond, C. T., Rovó, Z., Chatton, J. Y., Adelman, J. P., et al. (2012). Sustaining sleep spindles through enhanced SK2-channel activity consolidates sleep and elevates arousal threshold. J. Neurosci. 32, 13917-13928. doi: 10.1523/jneurosci.2313-12.2012

Winkler, S. A., Schmitt, F., Landes, H., de Bever, J., Wade, T., Alejski, A., et al. (2018). Gradient and shim technologies for ultra high field MRI. NeuroImage 168, 59-70. doi: 10.1016/j.neuroimage.2016.11.033

Woo, C. W., Schmidt, L., Krishnan, A., Jepma, M., Roy, M., Lindquist, M. A., et al. (2017). Quantifying cerebral contributions to pain beyond nociception. Nat. Commun. 8:14211. doi: 10.1038/ncomms14211

Wu, T., Grandjean, J., Bosshard, S. C., Rudin, M., Reutens, D., and Jiang, T. (2017). Altered regional connectivity reflecting effects of different anaesthesia protocols in the mouse brain. NeuroImage 149, 190-199. doi: 10.1016/j. neuroimage.2017.01.074

Xu, F., Liu, N., Kida, I., Rothman, D. L., Hyder, F., and Shepherd, G. M. (2003). Odor maps of aldehydes and esters revealed by functional MRI in the 
glomerular layer of the mouse olfactory bulb. Proc. Natl. Acad. Sci. U S A 100, 11029-11034. doi: 10.1073/pnas.1832864100

Xu, F., Schaefer, M., Kida, I., Schafer, J., Liu, N., Rothman, D. L., et al. (2005). Simultaneous activation of mouse main and accessory olfactory bulbs by odors or pheromones. J. Comp. Neurol. 489, 491-500. doi: 10.1002/cne.20652

Yli-Hankala, A., Lindgren, L., Porkkala, T., and Jantti, V. (1993). Nitrous oxidemediated activation of the EEG during isoflurane anaesthesia in patients. $\mathrm{Br}$. J. Anaesth. 70, 54-57. doi: 10.1093/bja/70.1.54

Yoshida, K., Mimura, Y., Ishihara, R., Nishida, H., Komaki, Y., Minakuchi, T., et al. (2016). Physiological effects of a habituation procedure for functional MRI in awake mice using a cryogenic radiofrequency probe. J. Neurosci. Methods 274, 38-48. doi: 10.1016/j.jneumeth.2016.09.013

Yuste, R. (2015). From the neuron doctrine to neural networks. Nat. Rev. Neurosci. 16, 487-497. doi: $10.1038 / \mathrm{nrn} 3962$

Záborszky, L., Gombkoto, P., Varsanyi, P., Gielow, M. R., Poe, G., Role, L. W., et al. (2018). Specific basal forebrain-cortical cholinergic circuits coordinate cognitive operations. J. Neurosci. 38, 9446-9458. doi: 10.1523/jneurosci.167618.2018

Zaman, T., Lee, K., Park, C., Paydar, A., Choi, J. H., Cheong, E., et al. (2011). Cav2.3 channels are critical for oscillatory burst discharges in the reticular thalamus and absence epilepsy. Neuron 70, 95-108. doi: 10.1016/j.neuron.2011. 02.042

Zandieh, S., Hopf, R., Redl, H., and Schlag, M. G. (2003). The effect of ketamine/xylazine anesthesia on sensory and motor evoked potentials in the rat. Spinal Cord 41, 16-22. doi: 10.1038/sj.sc.3101400

Zeman, A. (2004). Theories of visual awareness. Prog. Brain Res. 144, 321-329. doi: 10.1016/s0079-6123(03)14422-6

Zerbi, V., Floriou-Servou, A., Markicevic, M., Vermeiren, Y., Sturman, O., Privitera, M., et al. (2019). Rapid reconfiguration of the functional connectome after chemogenetic locus coeruleus activation. Neuron 103, 702.e5-718.e5. doi: 10.1016/j.neuron.2019.05.034

Zerbi, V., Grandjean, J., Rudin, M., and Wenderoth, N. (2015). Mapping the mouse brain with rs-fMRI: an optimized pipeline for functional network identification. NeuroImage 123, 11-21. doi: 10.1016/j.neuroimage.2015.07.090
Zhang, R. V., Featherstone, R. E., Melynchenko, O., Gifford, R., Weger, R., Liang, Y., et al. (2019). High-beta/low-gamma frequency activity reflects top-down predictive coding during a spatial working memory test. Exp. Brain Res. 237, 1881-1888. doi: 10.1007/s00221-019-05558-3

Zhang, S., Hu, S., Hu, J., Wu, P. L., Chao, H. H., and Li, C. S. (2015). Barratt impulsivity and neural regulation of physiological arousal. PLoS One 10:e0129139. doi: 10.1371/journal.pone.0129139

Zhang, S., Xu, M., Kamigaki, T., Hoang Do, J. P., Chang, W.-C., Jenvay, S., et al. (2014). Long-range and local circuits for top-down modulation of visual cortex processing. Science 345, 660-665. doi: 10.1126/science.12 54126

Zhang, Y., Yoshida, T., Katz, D. B., and Lisman, J. E. (2012). NMDAR antagonist action in thalamus imposes delta oscillations on the hippocampus. J. Neurophysiol. 107, 3181-3189. doi: 10.1152/jn. 00072.2012

Zhao, F., Zhao, T., Zhou, L., Wu, Q., and Hu, X. (2008). BOLD study of stimulation-induced neural activity and resting-state connectivity in medetomidine-sedated rat. NeuroImage 39, 248-260. doi: 10.1016/j. neuroimage.2007.07.063

Zhou, H., Zhang, Q., Martinez, E., Dale, J., Hu, S., Zhang, E., et al. (2018). Ketamine reduces aversion in rodent pain models by suppressing hyperactivity of the anterior cingulate cortex. Nat. Commun. 9:3751. doi: 10.1038/s41467018-06295-x

Conflict of Interest: The authors declare that the research was conducted in the absence of any commercial or financial relationships that could be construed as a potential conflict of interest.

Copyright (๑) 2020 Reimann and Niendorf. This is an open-access article distributed under the terms of the Creative Commons Attribution License (CC BY). The use, distribution or reproduction in other forums is permitted, provided the original author(s) and the copyright owner(s) are credited and that the original publication in this journal is cited, in accordance with accepted academic practice. No use, distribution or reproduction is permitted which does not comply with these terms. 